\title{
Polarized neutron reflectometry study of thin Fe films prepared on V(100)
}

\author{
vorgelegt von \\ Master of Science \\ Yuntao LIU \\ Aus China \\ von der Fakultät II - Mathematik- und Naturwissenschaften \\ der Technischen Universität Berlin \\ zur Erlangung des akademischen Grades \\ Doktor der Naturwissenschaften \\ - Dr.rer.nat. - \\ genehmigte Dissertation
}

Promotionsausschuss:

Vorsitzender: Prof. Dr. E. Sedlmayr

Berichter: Prof. Dr. H. Maletta

Berichter: Prof. Dr. C. Thomsen

Tag der wissenschaftlichen Aussprache: 29. April 2004

Berlin 2004

D83 


\section{Abstract}

The present work emphasizes on the polarized neutron reflectometry (PNR) method as a powerful tool to study details of the magnetization of an ultrathin film by combining in-situ and ex-situ PNR measurements as function of the film thickness for the first time. The aim is to separate and estimate the magnetization contributions from the interface, the inner part and the free surface of the film by investigating capped and uncapped films.

A series of samples of $\mathrm{V} / t_{\mathrm{Fe}} \mathrm{Fe} / \mathrm{V}(100)$ and $t_{\mathrm{Fe}} \mathrm{Fe} / \mathrm{V}(100)$ all grown on $\mathrm{MgO}(100)$ substrates with different Fe thickness $t_{\mathrm{Fe}}$ were prepared by molecular beam epitaxy (MBE). The critical part of the preparation of the $\mathrm{Fe} / \mathrm{V}$ samples was the preparation of a chemically clean Vanadium substrate layer with a good crystalline quality. Instead of using a V single crystal substrate, $\mathrm{V}$ was evaporated on a $\mathrm{MgO}(100)$ substrate together with an additional ultrathin V toplayer by MBE with various heat treatments. This procedure was successful leading to a smooth and clean V(100) surface with good crystalline quality. Concerning the samples for the ex-situ measurements it was established with the help of x-ray reflectometry (XRR) that $\mathrm{V}_{2} \mathrm{O}_{3}$ was formed on top of the $\mathrm{V}$ capping layer after the samples were exposed to air. The oxidation, however, did not penetrate as deep as to reach the $\mathrm{Fe} / \mathrm{V}$ interface which would have influenced the Fe magnetization.

The samples were studied using ex-situ PNR, ex-situ XRR, in-situ PNR, and in-situ magneto-optical Kerr effect (MOKE). The ex-situ measurements were done on V-capped samples, while uncapped Fe films were used in the in-situ measurements. According to the results obtained the product of the magnetic moment per atom and the Fe thickness of ultrathin $\mathrm{Fe}(100)$ films was plotted as a function of the Fe thickness. Analyzing these plots the different contributions to the magnetization were estimated. The slope of this kind of plot is correlated with the magnetization from the inner part of the Fe layer. On the other hand, the intersection at the abscissa may help to separate the contributions from the interface and free surface.

All the PNR data are consistent with the concept of a linear superposition of a volume and a surface term for describing the magnetization of a thin film, where the volume term grows linearly with the thickness. The experiments clearly indicate that the capping of the thin Fe film with $\mathrm{V}$ changes the total magnetization, whereas it does not affect the magnetic moment of the inner part of the Fe layer (which is that of bulk Fe). Combining both series of PNR experiments demonstrates the existence of a reduced magnetic moment at the $\mathrm{Fe} / \mathrm{V}$ interface, estimated to be a reduction of $-0.75( \pm 0.05) \mu_{\mathrm{B}} /$ atom which corresponds to about $35 \%$ of the Fe bulk value. In contrast, an enhanced magnetic moment at the free Fe surface is derived (estimated to be an enhancement of $+0.3( \pm 0.9) \mu_{\mathrm{B}} /$ atom).

Comparing the present results with the results obtained by Nawrath et al. [Naw99] and by Schmitz et al. [Sch04] on the Fe/V(110) system we conclude that the large reduction of the magnetic moment at the Fe/ $\mathrm{V}(110)$ interface as found by Nawrath et al. is not a generic case. 


\section{Zusammenfassung}

Die vorliegende Arbeit befasst sich insbesonders mit der experimentellen Methode der Reflektometrie mit polarisierten Neutronen (PNR). In einer Weiterentwicklung dieser wichtigen Technik wurden durch Kombination von in-situ und ex-site PNR-Messungen als Funktion der Schichtdicke zum ersten Mal damit Details der Magnetisierung eines ultradünnen Films untersucht. Es wird angestrebt, durch solche Untersuchungen an Schichten mit und ohne Abdeckschicht die Beiträge zur Magnetisierung von der Grenzfläche, dem Schichtinneren und der freien Oberfläche zu separieren und in ihrer Grösse abzuschätzen.

Reihen von Schichtproben von $\mathrm{V} / t_{\mathrm{Fe}} \mathrm{Fe} / \mathrm{V}(100)$ und $t_{\mathrm{Fe}} \mathrm{Fe} / \mathrm{V}(100)$ mit unterschiedlicher Fe-Schichtdicke $t_{\mathrm{Fe}}$ wurden auf $\mathrm{MgO}(100)$-Substrat mit MBE präpariert. Besonders kritisch dabei war die Präparation eines chemisch reinen Vanadium-Substrats mit guter KristallQualität. Statt einen V-Einkristall als Substrat zu benutzen, wurde V auf $\mathrm{MgO}(100)$ durch MBE-Epitaxie aufgedampft und unter verschiedenen Behandlungen schließlich mit einer ultradünnen V-Schicht abgedeckt. Diese Prozedur war erfolgreich und führte zu einer planen und reinen V(100)-Oberfläche mit guter kristalliner Qualität. An den ex-situ Messproben wurde mit Hilfe von XRR die Ausbildung von $\mathrm{V}_{2} \mathrm{O}_{3}$ an der Oberfläche der VAbdeckschicht festgestellt, nachdem sie der Luft ausgesetzt waren. Die Oxidation drang jedoch nicht bis zur Tiefe der Fe/V-Grenzschicht durch, was die Fe-Magnetisierung beeinflusst hätte.

Die Schichtproben wurden mit ex-situ PNR, ex-situ XRR, in-situ PNR, und in-situ MOKE untersucht, wobei die ex-situ-Messungen an Schichten mit V-Abdeckung durchgeführt wurden, und die in-situ-Messungen an Fe-Schichten mit freier Oberfläche. Alle Messdaten wurden in einem Plot dargestellt, mit dem Produkt aus magnetischem Moment mal Schichtdicke der Fe(100)-Filme als Funktion von der Fe-Schichtdicke. Durch die Auswertung dieser Plots wurden die verschiedenen Beiträge zur Magnetisierung abgeschätzt: Die Steigung der Geraden ist mit der Magnetisierung im Innern der Fe-Schicht korreliert; andererseits führt der x-Achsenabschnitt zur Separation der Beiträge von der Grenzfläche und der freien Oberfläche.

Alle PNR-Daten erlauben die Beschreibung der Magnetisierung einer dünnen Schicht in konsistenter Weise mit dem Konzept der linearen Superposition eines Volumen- und Oberflächenanteils, wobei der Volumenanteil linear mit der Schichtdicke anwächst. Die Ergebnisse zeigen deutlich, dass durch die V-Abdeckung die Gesamtmagnetisierung geändert wird, aber nicht das magnetische Fe-Moment im Innern der Fe-Schicht (das mit dem üblichen Moment von bulk-Fe übereinstimmt). Die Kombination der Daten aus beiden PNR-Messreihen zeigt einerseits die Existenz eines reduzierten magnetischen Momentes an der Fe/V-Grenzfläche, was zu einer Reduzierung um $-0.75( \pm 0.05) \mu_{\mathrm{B}} /$ Atom abgeschätzt wurde (entsprechend in etwa 35\% des bulk-Fe-Wertes). Im Gegensatz dazu zeigt sich ein erhöhtes magnetisches Moment an der freien Fe-Oberfläche, was zu einer Erhöhung von ca. $+0.3( \pm 0.9) \mu_{\mathrm{B}} /$ Atom abgeschätzt wurde.

Aus dem Vergleich unser Ergebnisse mit den Arbeiten von Nawrath et al. [Naw99] und Schmitz et al. [Sch04] an dem Fe/V(110)-System schließen wir, dass die in [Naw99] beobachtete starke Reduzierung der Magnetisierung an der Fe/V(110)-Grenzfläche keine allgemeine Gültigkeit hat. 


\section{Contents}

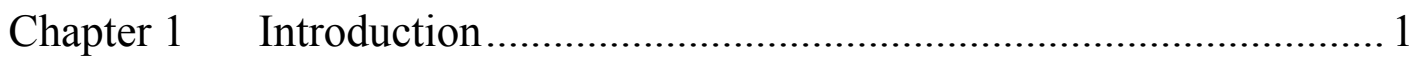

1.1 Some general aspects on thin films ................................................ 1

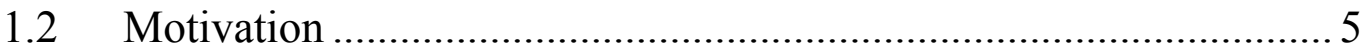

Chapter 2 Experimental Techniques.................................................... 7

$2.1 \quad$ Polarized Neutron Reflectometry …………………………….... 7

2.2 Magneto-optical Kerr effect ...................................................... 14

2.3 Low-energy electron diffraction................................................... 16

2.4 Auger Electron Spectroscopy ……………….............................. 20

Chapter 3 Sample Preparation ............................................................ 22

3.1 Basic models and principles of MBE .......................................... 22

3.2 Experimental setup of the MBE chamber ...................................... 24

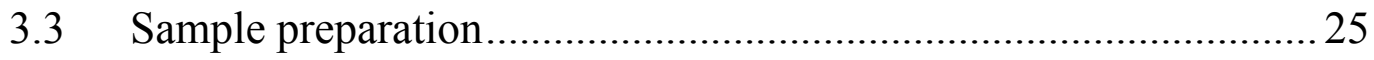

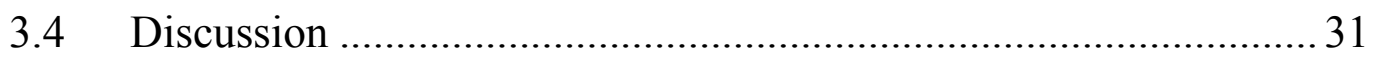

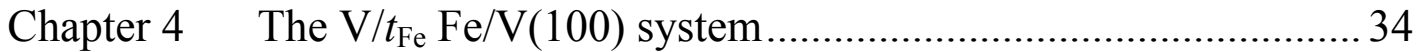

4.1 Measurements at room temperature ……………........................... 34

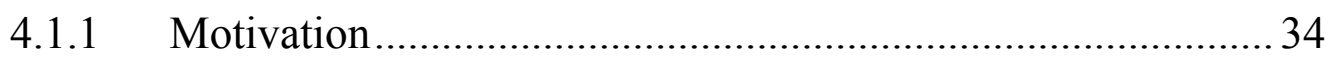

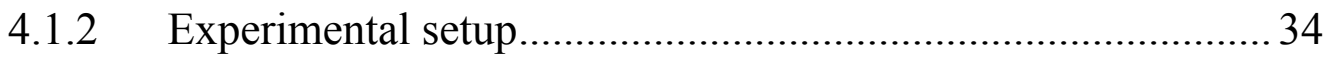

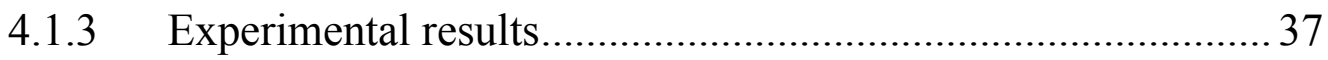

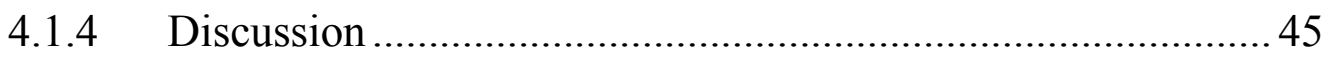

4.2 Temperature-dependent PNR measurements ................................... 49

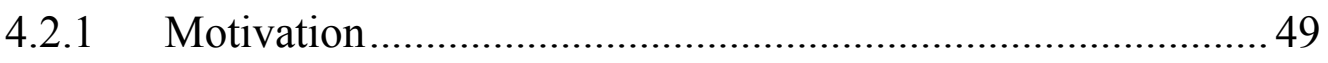

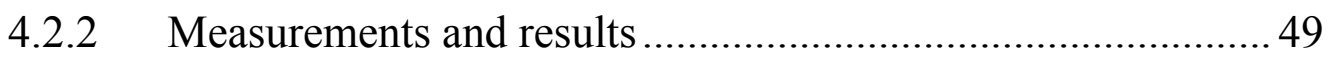

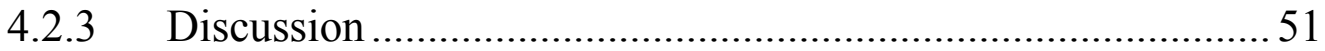




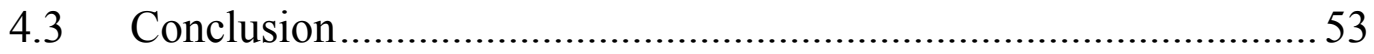

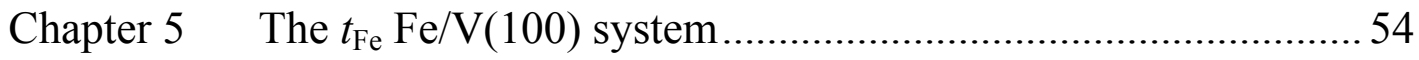

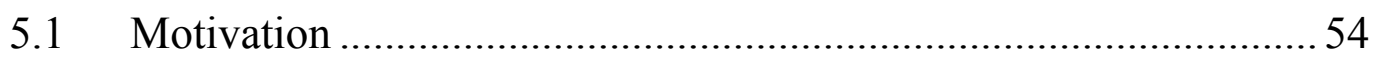

5.2 In-situ MOKE measurements ....................................................... 55

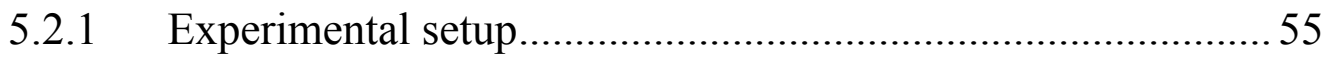

5.2.2 Measurements and results ....................................................... 56

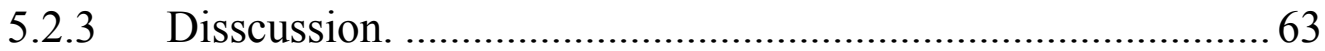

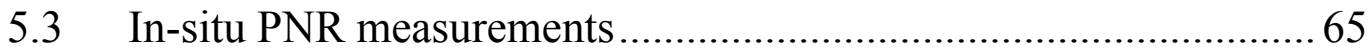

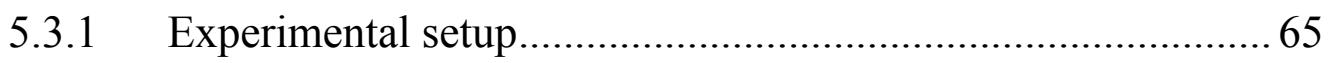

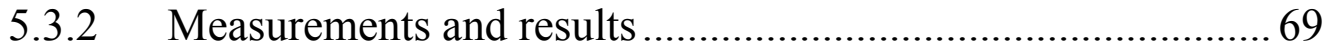

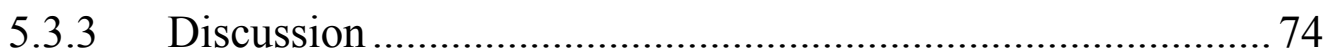

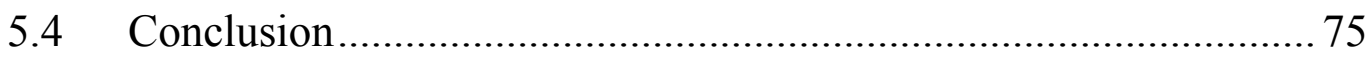

Chapter $6 \quad$ Summary ………………................................................... 78 


\section{Chapter 1 Introduction}

\subsection{Some general aspects on thin films}

Research on thin (i.e. nm lengthscale) metallic films was already a center of activity in magnetism in the late 1950s, motivated by the prospect of discovering novel magnetic phenomena with potential for application. It was anticipated from theoretical considerations that the reduced thickness and the presence of the interface might significantly influence the magnetic properties of the films.

The possibility of "magnetically dead layers" at the surface of ferromagnetic Fe and Ni was announced by Lieberman et al. [Lie69, Lie70]. Although later it was proven that the phenomenon of "magnetically dead layers" arised due to contamination, it has stimulated great interest in studying magnetic properties of surfaces and thin films. The follow-up experiments on various ultrathin films have given clear evidence that ferromagnetic order does exist even in monolayer films.

The existence of ferromagnetic order in ultrathin films obviously is great news for scientists who are interested in low-dimensional magnetism. In the past two decades, magnetic ultrathin films have been extensively studied and some striking differences between two-dimensional (2D) ultrathin films and three-dimensional (3D) bulk crystals have been observed as shown below.

\section{Magnetic anisotropy}

One of the most remarkable properties in a 2D system is given by its unusual magnetic anisotropies. The surface atoms will contribute differently to 
the anisotropy energy since the local symmetry of these atoms is different from that in the bulk.

Following the distinctions made by Gradmann [Gra93] the magnetic anisotropies in homogeneously magnetized samples or homogeneously magnetized regions of a film can be categorized as:

- Magnetostatic anisotropy (Shape anisotropy)

- Magnetocrystalline anisotropy

- Magnetic surface anisotropy

- Strain anisotropy

The thickness of thin films is small in comparison with any characteristic length which is relevant for changes of the magnetization direction. A single domain equilibrium results and the magnetization direction lies in the film plane. This tendency towards in-plane magnetization is due to Magnetostatic anisotropy (Shape anisotropy) since it is originated from the slab shape of thin films.

For a magnet with crystallographic symmetry, there exists an energy term called Magnetocrystalline anisotropy, which favors magnetization directions along certain major crystal symmetry axes. Néel [Née54] was the first to note that the dramatic break of local magnetic symmetry in a surface must result in strong surface-type anisotropies. The Magnetocrystalline anisotropy at the surface is generally one or two orders of magnitude larger than that of the bulk. This new energy term is often referred to as Magnetic surface anisotropy, which may favor magnetization direction either parallel or perpendicular to the film plane depending on the system. In case the surface anisotropy favors perpendicular magnetization and is large enough to overcome the in-plane shape anisotropy, the magnetization direction of the film will be perpendicular to the film plane [She02]. Such perpendicular magnetization has been observed in numerous magnetic ultrathin film and multilayer systems [Car85, Eng91, Gra68].

Strain, caused, for example, by different thermal expansions of the film and the substrate, or by defects induced by the growth process itself, often occurs in epitaxial films. The anisotropy related to strain is called Strain anisotropy. In particular for pseudomorphic films, the Strain anisotropy can be very strong. 


\section{Magnetic moment in thin films}

At low temperatures $T / T_{c} \leq 0.4$ the magnetic moment $m(\mathrm{~T})$ of a magnetically saturated bulk crystal is given by Bloch's law

$$
m_{\text {bulk }}(T)=m_{b u l k}(0) \cdot\left(1-b T^{\frac{3}{2}}\right)
$$

where $m_{\text {bulk }}(0)$ is the magnetic moment of the bulk crystal at $T=0 \mathrm{~K}$ and the spin wave parameter $b$ is a material dependent constant.

The spin wave parameter $b$ accounts for the effect of the reduced thickness in a thin film [Gra93] as follows:

$$
b=b_{v}+\frac{1}{t} b_{s}
$$

where $b_{v}$ is the volume term, which is not influenced by the layer thickness, $b_{s}$ is a term that determines how the film thickness influences the spin wave parameter $b$ [Fri95], $t$ is the film thickness. Then Eq. (1-1) can be written as

$$
\begin{aligned}
m(T, t) & =m_{\text {bulk }}(T)-m_{\text {bulk }}(0) \cdot \frac{1}{t} b_{s} T^{\frac{3}{2}} \\
& =m_{\text {bulk }}(T)+\Delta m_{\text {size }}(T),
\end{aligned}
$$

At the surface the reduced coordination number causes a decrease of the band width (Figure 1.1) [Sch97], more majority states become populated and therefore the weak magnetic moment increases at the surface. At the surface of the crystal, the spin waves are reflected. Since the surface is a free end, the spin waves of any wavelength will exhibit an antinode at the surface layer [Sie92].

Thus, accounting for the influence of both, the thickness and the surface of the thin film, the product of the magnetic moment of the film and its thickness $t$ can be expressed as

$$
m(T, t)=m_{\text {bulk }}(T, t)+\Delta m_{\text {size }}(T)+\Delta m_{\text {surface }} .
$$




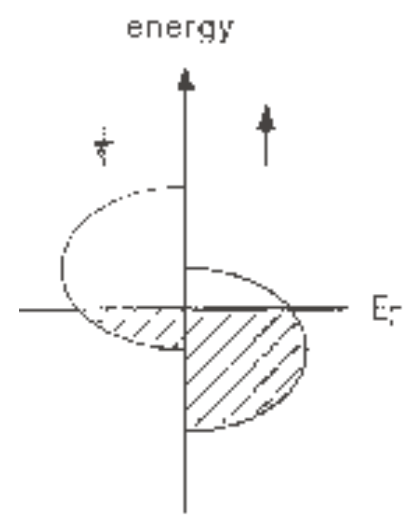

bulk

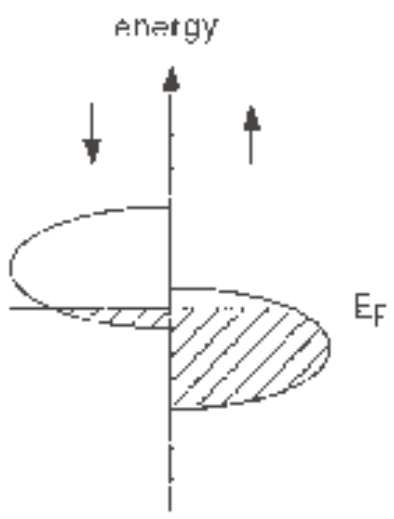

surface

Figure 1.1 Schematic density of states of a weak ferromagnet. The arrows denote the electron spin. At the surface the reduced coordination number causes a band narrowing relative to the bulk. Thus more majority spin states become occupied and the magnetic moment increase. [Sch97]

\section{Correlation between structure and magnetism}

The magnetic properties and the structural properties of 2D ultrathin films are strongly correlated. Magnetic quantities such as the magnetic moment, the magnetic anisotropy and the Curie temperature are closely linked to structural parameters such as the lattice constant, strain, roughness and intermixing between film and substrate etc.

For example, in contrast to the open character of the (100) surface with four nearest neighbors for each surface atom, the (110) surface has a higher surface packing density, i.e., six nearest neighbors for each surface atom. Using the full-potential linearized augmented-plane-wave method (FLAPW), Freeman et al. [Fre85, Fre87] have investigated the magnetic properties of transition metal surfaces by solving the local spin density functional equations. The calculated value of $2.98 \mu_{\mathrm{B}}$ for the Fe magnetic moment at the (100) surface is larger than the value of $2.63 \mu_{\mathrm{B}}$ for the magnetic moment at the (110) surface. This nicely fits in the simple picture that the magnetic moment is in inverse proportion to the number of nearest neighbors. 


\section{The Fe/V system}

In recent years induced magnetic ordering at the interface between a nonmagnetic and a magnetic material has attracted considerable interest. The $\mathrm{Fe} / \mathrm{V}$ system is one of them. It has been investigated by a number of experimental [Chr95, Dud96, Fri98, Fuc96, Gra98, Har95, Hos84, Ige00, Naw98, Pou97, Sch01, Tom97, Wa194] and theoretical [Alv98, Coe95, Ham84, Izq99, Izq01, Mar95, Mos98, Spi00, Veg91, Veg93] works. In these experimental works, using x-ray magnetic circular dichroism, Harp et al. observed that for a sample $0.44 \mathrm{~nm} \mathrm{Fe} / 0.3 \mathrm{~nm} \mathrm{~V}$ multilayer with the (100) orientation, the total $\mathrm{Fe}$ magnetic moment was near that of bulk $\mathrm{Fe}$ and the magnetic moment of $\mathrm{V}$ was larger than $0.26 \mu_{\mathrm{B}}$, aligned antiferromagnetically with the Fe. But the difference of the polarization values found by the different authors amounts to $50 \%$. The measurements presented in these studies were either performed in-situ in an ultrahigh vacuum chamber by methods, which do not measure the absolute magnitude of the magnetization, or the authors prepared multilayered structures and determined the magnetization at the interfaces by magnetic ex-situ experiments.

Polarized Neutron Reflectometry (PNR) has been proven to be a very powerful technique for the investigation of magnetic and structural properties of thin magnetic films and multilayers. It can be used to measure the absolute magnetic moment and unlike SQUID it does not suffer from huge diamagnetic contributions of the substrate. For neutrons the nuclear scattering length of V is nearly zero, so PNR is especially suitable for the investigation of the magnetic moment of ultrathin Fe films prepared on V. That was already proven in recent experiments on $\mathrm{Fe}(110)$ films performed by Nawrath et al. [Naw00, Naw99].

\subsection{Motivation}

As mentioned above, the films prepared on substrates with different orientation are expected to show different magnetic properties, due to the different local environment (lattice constants, the number of the nearest neighbor atoms, etc.). T. Nawrath [Naw99] investigated the Fe/V(110) system and observed a large magnetization reduction at the $\mathrm{Fe} / \mathrm{V}(110)$ interface by in-situ PNR. In order to find out whether this reduction is a generic feature of the $\mathrm{Fe} / \mathrm{V}$ system this work on the $\mathrm{Fe} / \mathrm{V}(100)$ system was initiated. By combining 
the results obtained by ex-situ PNR and in-situ PNR the magnetic properties of the free Fe surface, the inner part of the Fe layer and the interface of the $\mathrm{Fe} / \mathrm{V}(100)$ system have been investigated. This work is organized as follows:

- In chapter two the main experimental techniques used in this work will be introduced briefly;

- In chapter three the sample preparation will be described;

- In chapter four the results obtained from ex-situ PNR and XRR on $\mathrm{V}$-covered Fe layers deposited on V(100) will be presented;

- In chapter five the results of uncovered Fe layers on V(100) will be shown;

- In chapter six the summary of this work will be presented. 


\section{Chapter 2 Experimental Techniques}

\subsection{Polarized Neutron Reflectometry}

PNR has been proven to be a very powerful technique for the investigation of magnetic and structural properties of thin magnetic films and multilayers [Bla94, Dob94, Fer99, Gie02, Naw99, We199, Zab94]. As a function of the glancing incident angle, the in-plane average of the scattering density can be deduced at any specified distance along the normal of the surface by counting the number of neutrons elastically and specularly reflected from a flat surface. From this scattering length density profile, the concentration of atoms of a given type at a particular depth can be inferred. Furthermore, if the incident beam is polarized and the resultant polarization of the reflected beam is analyzed, possible magnetic contributions to the scattering density can be distinguished in microscopic detail [Maj99].

In order to understand the working principle of PNR, a short introduction to the technique and its physical background will be given in the following paragraphs.

\section{Simple non-magnetic systems}

The neutron can be described by a plane wave vector $\vec{k}$, whose magnitude is $k=2 \pi / \lambda$, and its kinetic energy $E_{k i n}=\frac{\hbar^{2} k^{2}}{2 m}$. The propagation of a neutron wave can be expressed by the time independent Schrödinger equation 


$$
-\frac{\hbar^{2}}{2 m} \frac{\mathrm{d}^{2} \Psi(\overrightarrow{\mathrm{r}})}{\mathrm{d}^{2}}+V(\overrightarrow{\mathrm{r}}) \Psi(\overrightarrow{\mathrm{r}})=E(\overrightarrow{\mathrm{r}}) \Psi(\overrightarrow{\mathrm{r}})
$$

where $V$ and $E$ are the potential and total energy of the neutron respectively. If the neutron wave is reflected elastically by a perfect planar boundary between medium A and B (see Figure 2.1), then $k_{\mathrm{i}}=k_{\mathrm{r}}$ and $q=2 k_{\mathrm{i}} \sin \theta_{\mathrm{i}}$ ( $q$ is the magnitude of the wavevector transfer) are valid. Usually the total energy is the sum of the kinetic and potential energies, and the kinetic energy is $\hbar^{2} k^{2} /(2 m)$, so that Eq. (2-1) can be transformed into

$$
\frac{d^{2} \Psi(\vec{r})}{d \vec{r}^{2}}+k^{2}(\overrightarrow{r)} \Psi(\vec{r})=0
$$
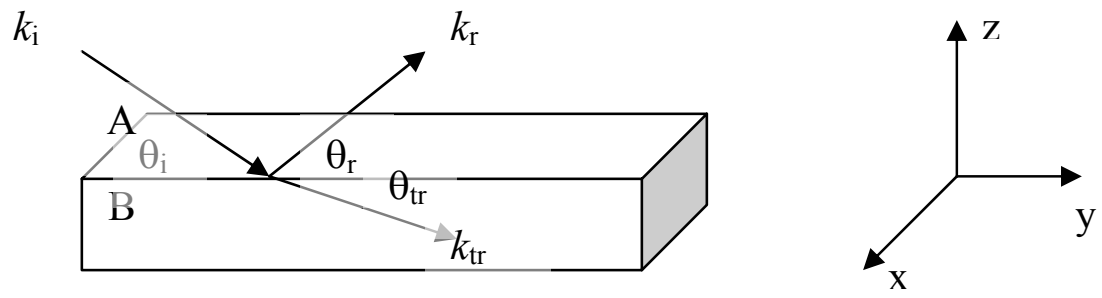

Figure 2.1 Scattering configuration for reflectivity studies. $k_{i}, k_{r}$ and $k_{t r}$ are the incident, reflected and transmitted wavevector, $\theta_{i}, \theta_{r}$ and $\theta_{t r}$ are the angles of these beams relative to the surface between medium $A$ and medium $B$, respectively.

For simplicity, let medium A be air and the potential energy in medium B be constant. According to the energy conservation law the energy balance can be expressed as 


$$
\frac{\hbar^{2} k_{i}^{2}}{2 m}=\frac{\hbar^{2} k_{t r}^{2}}{2 m}+V .
$$

If $q$ is sufficiently small, so that the magnitude of $2 \pi / q$ is much greater than the interatomic distances in the medium, the medium can be treated as if it was continuum, though the density of the medium need not be constant along the direction parallel to $\vec{q}$. In this continuum limit, the potential energy $V$ can be expressed as (magnetic contributions are neglected for the time being)

$$
V=\frac{2 \pi \hbar^{2}}{m}<\sum_{i} N_{i} b_{i}>=\frac{2 \pi \hbar^{2}}{m} \rho .
$$

$N_{\mathrm{i}}$ and $b_{\mathrm{i}}$ are the number density and coherent scattering length of species i, $\langle\cdots\rangle$ denotes the average and $\rho$ is called the scattering length density. Neutron absorption is taken into account by the imaginary part of the scattering length $b=b^{\prime}+\mathrm{i} b^{\prime \prime}$ with $b^{\prime \prime}=k \sigma_{a}(k) /(4 \pi)$, where $\sigma_{a}$ represents the absorption cross section. But except for very thick layers and/or strongly absorbing elements, such as $\mathrm{Cd}$ and $\mathrm{Gd}$, the absorption can be neglected. Substituting Eq. (2-4) into Eq. (2-3) it can be transformed into

$$
k_{t r}^{2}=k_{i}^{2}-4 \pi \rho
$$

Where $k_{\mathrm{i}}$ and $k_{\text {tr }}$ are the wave vectors of the incident and transmitted waves.

The neutron refractive index $n$ can be written as

$$
n=\frac{k_{t r}}{k_{i}}=\sqrt{1-\frac{4 \pi \rho}{k_{i}^{2}}} .
$$

At the interface the Snell's law holds

$$
\cos \theta_{i}=n \cos \theta_{t r}
$$

then the critical angle of total reflection can be given as follows

$$
\cos \theta_{c}=n=\sqrt{1-\frac{4 \pi \rho}{k_{i}^{2}}} .
$$


Since $\theta_{\mathrm{c}}$ is very small for neutrons it is possible to use a Taylor expansion. Using Eq. (2-8) the expression of $\theta_{\mathrm{c}}$ can be transformed into

$$
\theta_{c}=\sqrt{\frac{\rho}{\pi}} \lambda .
$$

The corresponding critical wave vector is

$$
q_{c}=\frac{4 \pi \sin \theta_{c}}{\lambda}=4 \sqrt{\pi \rho} .
$$

One simplification of the equation of motion, Eq. (2-1), can be made if we assume no lateral variation of the scattering density. The interaction with the reflecting medium can be expressed by a one dimensional effective potential $V(\mathrm{z})$ leading to the one dimensional Schrödinger equation

$$
\frac{d^{2} \Psi(z)}{d z^{2}}+k(z)^{2} \Psi(z)=0 .
$$

At the interface $\psi$ and $\nabla \psi$ have to fulfill the continuity condition. It is possible to show that the parallel components of the incident and reflected waves are continuous. The continuity of the parallel components allows us to write

$$
k_{t r z}^{2}=\frac{q^{2}}{4}-4 \pi \rho,
$$

where $k_{\text {trz }}$ represents the $\mathrm{z}$ component of the wave vector of the transmitted wave. According to the Fresnel law for $\mathrm{q}>\mathrm{q}_{\mathrm{c}}$ the reflectivity is given by [Fer99]

$$
R=\left|\frac{\frac{q}{2}-k_{t r z}}{\frac{q}{2}+k_{t r z}}\right|^{2}=\left|\frac{q-\sqrt{q^{2}-16 \pi \rho}}{q+\sqrt{q^{2}-16 \pi \rho}}\right|^{2} .
$$

If the wavevector transfer is very large compared to $q_{\mathrm{c}}$, i.e. $q \geq 3 q_{\mathrm{c}}$, the reflectivity approaches the asymptotic form 


$$
R \approx \frac{q_{c}^{4}}{16 q^{4}}
$$

If medium $\mathrm{A}$ is not air, $\rho$ in Eq. (2-13)can be replaced by $\left(\rho_{\mathrm{B}}-\rho_{\mathrm{A}}\right)$. Here $\rho_{\mathrm{A}}$ and $\rho_{\mathrm{B}}$ are the scattering length densities of medium $\mathrm{A}$ and medium $\mathrm{B}$ respectively.

\section{Simple magnetic systems}

Now, assuming that medium B is magnetic and the neutron spin is aligned either parallel or anti-parallel to the magnetic induction $\vec{B}$ (the collinear case) in the sample, similar to the interaction of the neutron with the nuclei, a 'magnetic scattering length density' $\rho_{\mathrm{m}}$ can be defined as follows

$$
\rho_{m}= \pm<N_{i} p_{i}>
$$

where the 'magnetic scattering length' $p_{\mathrm{i}}$ is defined as $p_{\mathrm{i}}=\mathrm{C} \mu_{\mathrm{i}}$ and $\mu_{\mathrm{i}}$ is the magnetic moment of species $i$ in units of the Bohr magneton $\mu_{\mathrm{B}}$. The constant is given by $C=m \mu_{\mathrm{n}} \mu_{B} \mu_{0} /\left(2 \pi \hbar^{2}\right)=2.699 \mathrm{fm} / \mu_{B}$. The plus (minus) sign refers to a spin direction parallel (anti-parallel) to the magnetic induction in the sample. Then the potential energy can be expressed by

$$
V_{B}=\frac{2 \pi \hbar^{2}}{m} \rho_{n}-\overrightarrow{\mu_{n}} \cdot \vec{B}=\frac{2 \pi \hbar^{2}}{m}\left(\rho_{n} \pm \rho_{m}\right)
$$

where $\rho_{\mathrm{n}}$ is 'nuclear scattering length density'. If $\rho$ is replaced by $\rho_{n} \pm \rho_{m}$ in Eq.(2-10) and Eq.(2-13), the spin-up, spin-down reflectivities $\mathrm{R}^{+}, \mathrm{R}^{-}$and the critical wave vectors $q_{c}{ }^{ \pm}$for spin-up(+) and spin-down(-) can be expressed as

$$
R^{ \pm}=\left|\frac{q-\sqrt{q^{2}-16 \pi\left(\rho_{n} \pm \rho_{m}\right)}}{q+\sqrt{q^{2}-16 \pi\left(\rho_{n} \pm \rho_{m}\right)}}\right|^{2},
$$

and

$$
q_{c}^{ \pm}=4 \sqrt{\pi\left(\rho_{n} \pm \rho_{m}\right)} .
$$


In PNR measurements the spin asymmetry $P$ is a very important quantity. It is defined by

$$
P=\frac{R^{+}-R^{-}}{R^{+}+R^{-}},
$$

where $R^{+}\left(R^{-}\right)$represents the reflectivity of the spin up (spin down) neutrons. The spin asymmetry allows one to directly compare the reflectivity difference between spin up and spin down neutrons, which exists due to the spin dependent magnetic interaction.

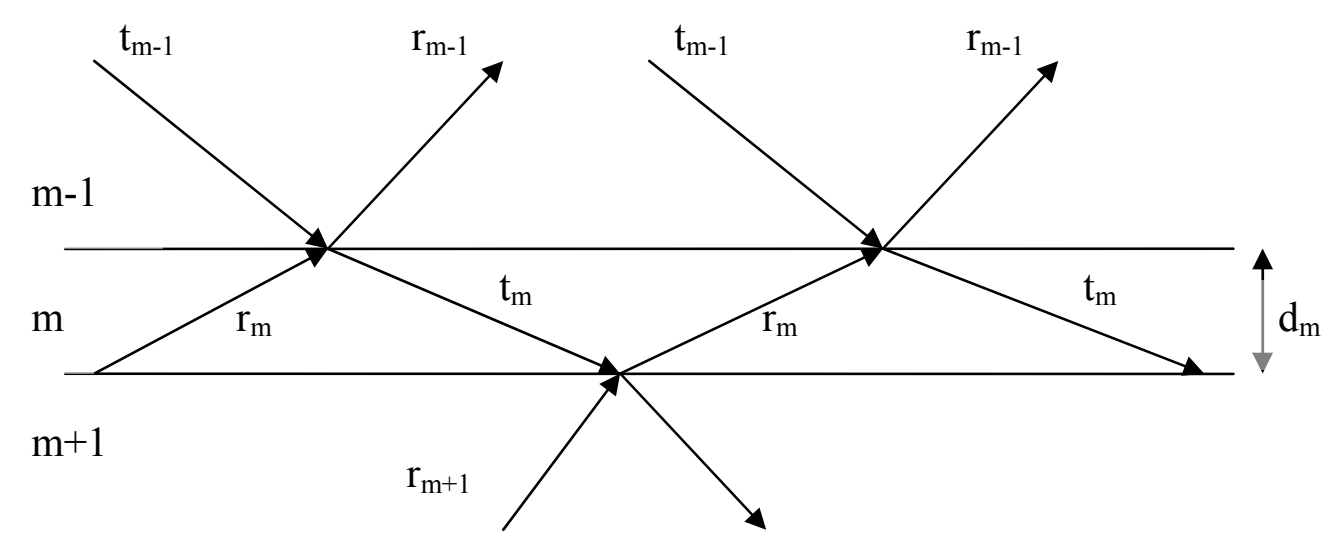

Figure 2.2 Reflection and transmission of waves occur at all interfaces of the different media in a layered sample.

\section{Multi-layer Systems}

In the case of thin films we have to deal with several interfaces. At each individual interface the boundary conditions must be fulfilled and for a stratified medium the total reflectivity is composed of the contributions from each interface. The Parratt formalism [Par54] is one method to calculate the reflected intensity of a stratified medium. It will be introduced briefly in the following paragraph. 
In Figure $2.2 r_{\mathrm{m}}$ and $t_{\mathrm{m}}$ are the amplitudes of the reflected and the transmitted waves in the $\mathrm{m}^{\text {th }}$ medium. $a_{m}=e^{i q_{m} d_{m} / 2}$ is a phase factor that is defined at the middle of the two surfaces of the $\mathrm{m}^{\text {th }}$ medium. $q_{\mathrm{m}}$ is the scattering vector in the $\mathrm{m}^{\text {th }}$ layer, and $d_{\mathrm{m}}$ is its thickness. According to the continuity condition of the transverse components of the wave vector the following expression of the Fresnel reflectivity for the interface between the $(m-1)^{\text {th }}$ and the $\mathrm{m}^{\text {th }}$ layer is obtained.

$$
R_{m-1}=a_{m-1}^{4}\left(\frac{R_{m-1, m}+R_{m}}{R_{m-1, m} R_{m}+1}\right)
$$

where

$$
\begin{aligned}
& R_{m}=\frac{r_{m}}{t_{m}} a_{m}^{2} \\
& R_{m-1, m}=\frac{q_{m-1}-q_{m}}{q_{m-1}+q_{m}}
\end{aligned}
$$

In order to derive the reflected intensity of a stratified medium, the Parratt formalism uses a recursion method starting from the lowest layer, which has to be thick enough, so that its Fresnel coefficient $R$ can be assumed to be zero, then counting the contribution of each layer backwards up to the top surface, where the reflected intensity is given by $I_{R} / I=\left|R_{0}\right|^{2}$.

The Parratt formalism has the advantage of providing the correct expression for all regions of scattering since no approximation is applied, since absorption is automatically taken into account, and since any density profile can be modeled by slicing the material in an arbitrary number of thin layers [Zab94].

The working principle of $\mathrm{x}$-ray scattering is similar to that of neutron scattering without considering the magnetic contribution. 


\subsection{Magneto-optical Kerr effect}

Magneto-optical effects arise from an optical anisotropy induced in diamagnetic materials by an externally applied magnetic field $H$, in para-, ferro-, and ferrimagnetic materials the origin of the anisotropy is the magnetization $M$. In general these effects change the state of incident linearly polarized light into rotated, elliptically polarized light after reflection from or transmission through the sample [Ned85]. The change in polarization of the reflected light arising due to magnetic interaction is called magneto-optical Kerr effect (MOKE). Its general property is that all manifestation of the Kerr effect are proportional to the magnetization $M(\mathrm{~T})$ and vanishes at temperatures above the Curie temperature [Bad94]. Nowadays MOKE is a very important tool in order to probe the magnetic properties of thin films.

Depending on the relative orientation of the magnetization vector $\vec{M}$ with respect to the incidence plane, three configurations are distinguished, as indicated in Figure 2.3. In the polar case the magnetization vector is perpendicular to the surface plane. In the longitudinal case the magnetization vector lies in the surface plane and parallel to the plane of incidence. In the transverse case the magnetization also lies in the surface plane but perpendicular to the incidence plane.

The main components of a MOKE spectrometer are shown in Figure 2.4. In the limit for ultrathin films $\left(\frac{2 \pi}{\lambda}|N| d<<1\right.$, where $d$ is the thickness of the films, $\lambda$ is the wavelength of the polarized light and $N$ is the refractive index of the films) and for the case of the longitudinal Kerr effect the Kerr rotation angle can be expressed as [Bad94]

$$
\phi=\left(\frac{4 \pi}{\lambda}\right)\left(\frac{N_{s u b}}{1-N_{s u b}^{2}}\right) \alpha \cdot Q \cdot t
$$

where $\phi$ is the Kerr rotation angle, $\alpha$ is the angle of incidence relative to the surface normal, $Q$ is the magneto-optical Voigt constant, $N_{\text {sub }}$ is the refractive index of the magnetic layer, and $t$ is the thickness of a magnetic layer. Due to the magneto-optical Kerr effect the intensity detected also changes. The relationship is given as follow: 


$$
I=I_{o r} \sin ^{2}\left(\gamma_{a}+\phi\right)+I_{r r}
$$

where $I_{0 \mathrm{r}}$ is the intensity of the reflected light, $I_{\mathrm{rr}}$ is the residual intensity transmitted through the analyzer when set for minimum transmission, and $\gamma_{\mathrm{a}}$ is the offset angle of the analyzer.

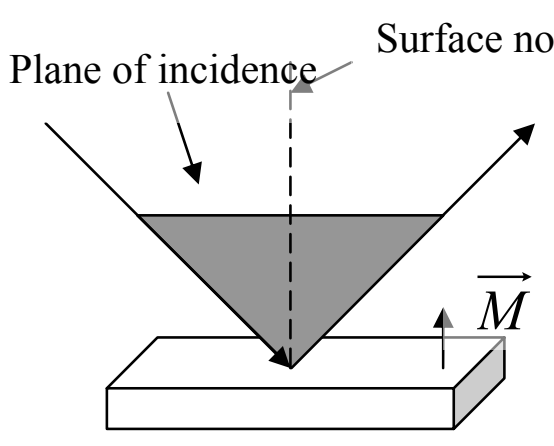

a.

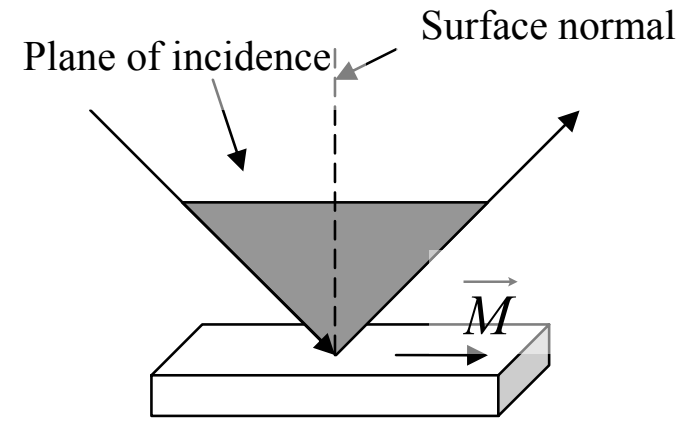

b.

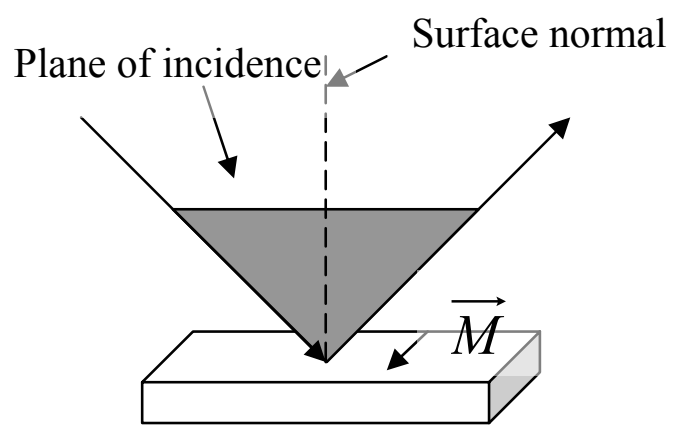

c.

Figure 2.3 Three magneto-optic Kerr effect configurations. (a) Polar case (b) Longitudinal case (c)Transverse case

In order to measure a hysteresis loop using the MOKE spectrometer, the detected intensity $I$ should be proportional to the magnetization $M$. Therefore, it is necessary to set $\gamma_{\mathrm{a}}>\phi$. In practice, both $\phi$ and $\gamma_{\mathrm{a}}$ are very small, i.e. $\sin ^{2}\left(\gamma_{a}+\phi\right) \sim\left(\gamma_{a}+\phi\right)^{2}$, i.e. 
MOKE is a technique that can probe the magnetic properties of a thin film at a relatively small region. This turns it into a good method in order to measure wedge-shaped samples, in which quantities depending on the layer thickness can be measured in a single sample. At the same time the effects of variations of other growth parameters are eliminated. Thus we can prepare a wedge-shaped sample to check how the magnetization changes with the layer thickness.

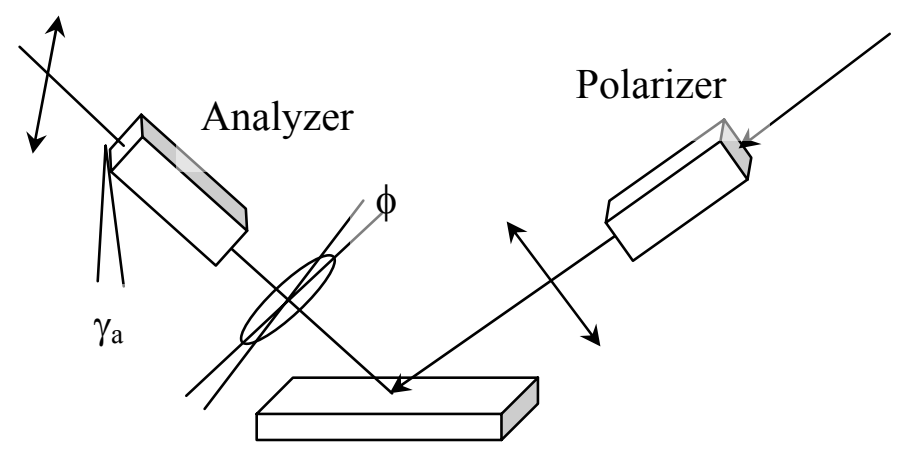

Figure 2.4 Schematic representation of a magneto-optic Kerr effect spectrometer: $\gamma_{a}$ is the analyzer off-set angle, and $\phi$ is the magnetooptic Kerr effect rotation

\subsection{Low-energy electron diffraction}

Low-energy electron diffraction (LEED) is a very powerful technique used for studying single crystal surfaces [Cla85, Ert85, Hov86, Nix]. Two types of information can be obtained from a LEED experiment:

- Firstly, it is possible to determine the structure of a periodic surface. The spot pattern indicates the surface order and its symmetry reflects the symmetry of the surface.

- Secondly, the intensities of the various diffracted beams can be recorded as a function of the incident electron beam energy. Comparing 
the latter with the theoretical curves, detailed information about the atomic positions may be obtained.

\section{Instrumentation}

The essential requirements for a LEED experiment are an electron gun, which produces a sufficiently parallel and mono-energetic electron beam with energies typically varying between 20 and $500 \mathrm{eV}$, and a detection system to record and display the diffracted beam intensity. A typical experimental setup is shown in Figure 2.5.

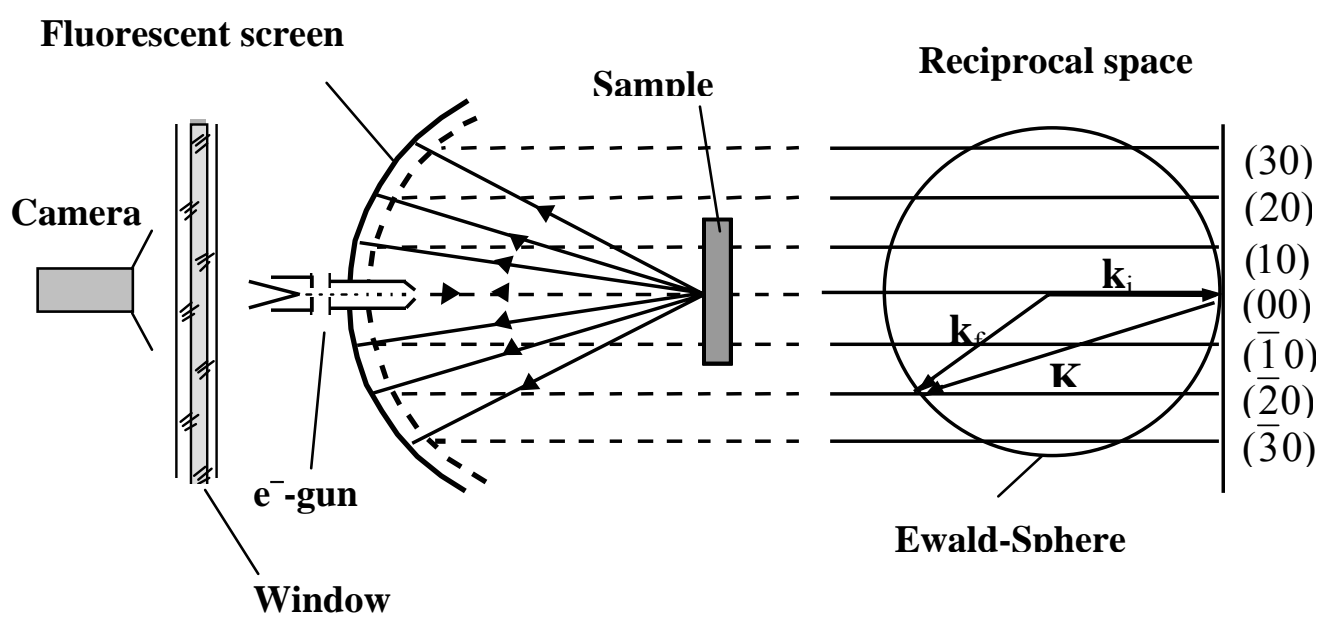

Figure 2.5 Sketch of a typical experimental LEED setup [Se101].

Electrons emitted from a heated cathode pass through a drift tube with the desired energy, and then strike the sample surface. Finally the backscattered electrons pass through a system of hemispherical concentric grids and hit a fluorescent screen, where diffraction spots will appear at the positions of the interference maxima. The LEED picture can be recorded using a CCD camera. 


\section{Basic theory of LEED}

A two dimensional crystal surface contains a primitive unit cell, which is defined by the translation vectors $\overrightarrow{a_{1}}$ and $\overrightarrow{a_{2}}$ (see Figure 2.6). Associated with this two dimensional unit cell is a unit cell in reciprocal space, which is defined by the translation vectors ${\overrightarrow{a_{1}}}^{*}$ and ${\overrightarrow{a_{2}}}^{*}$. The relationship between them are [Hov86]

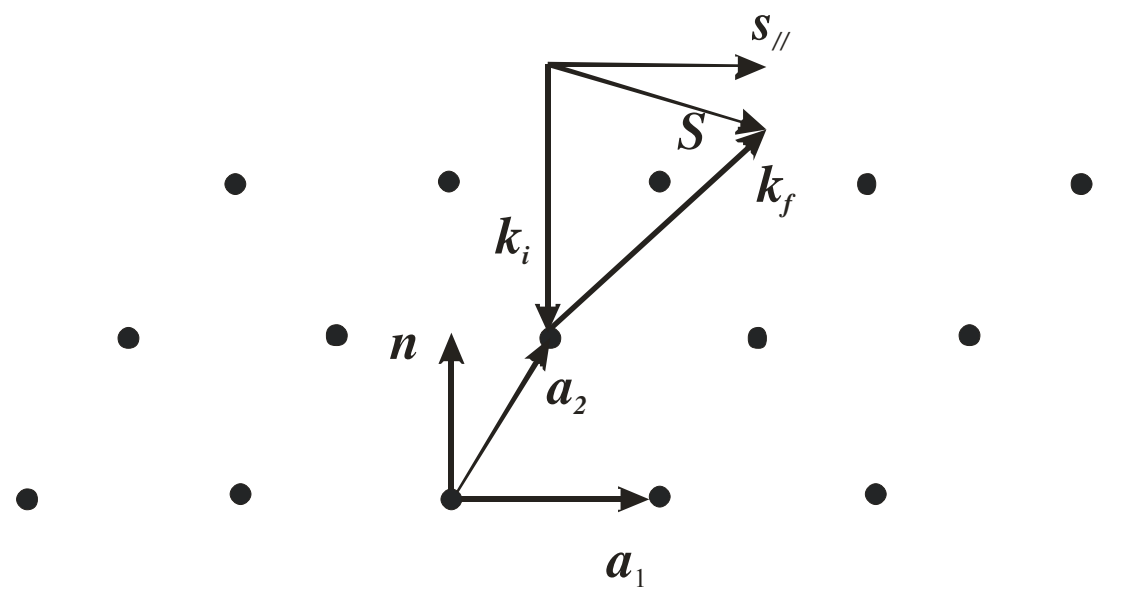

Figure 2.6 Diffraction of low-energy electrons by a two-dimensional crystal surface. $\boldsymbol{a}_{1}$ and $\boldsymbol{a}_{2}$ are the basis vectors of the lattice, $\boldsymbol{n}$ is a unit vector normal to and pointing out of the surface. $\boldsymbol{k}_{i}$ is the incident wave vector, $\boldsymbol{k}_{f}$ is the scattered wave vector, $\boldsymbol{s}_{/ /}$is the component of $\boldsymbol{k}_{i}-\boldsymbol{k}_{f}$ parallel to the surface.

$$
\begin{aligned}
& {\overrightarrow{a_{1}}}^{*}=2 \pi\left[\frac{\overrightarrow{a_{2}} \times \vec{n}}{\overrightarrow{a_{1}} \cdot\left(\overrightarrow{a_{2}} \times \vec{n}\right)}\right] \text {, and } \\
& {\overrightarrow{a_{2}}}^{*}=2 \pi\left[\frac{\vec{n} \times \overrightarrow{a_{1}}}{\overrightarrow{a_{2}} \cdot\left(\vec{n} \times \overrightarrow{a_{1}}\right)}\right],
\end{aligned}
$$

where $\vec{n}$ is a unit vector normal to and pointing out of the surface. From these relations, it is clear that $\overrightarrow{a_{1}} \cdot{\overrightarrow{a_{1}}}^{*}=\overrightarrow{a_{2}} \cdot{\overrightarrow{a_{2}}}^{*}=2 \pi$ and $\overrightarrow{a_{1}} \cdot \vec{a}_{2}^{*}=\overrightarrow{a_{2}} \cdot \vec{a}_{1}^{*}=0$ are satisfied [Hov86]. 
The incident beam of electrons can be presented by a plane wave

$$
\Psi_{i}=\Psi_{0} \cdot \exp \left(\overrightarrow{k_{i}} \cdot \vec{r}\right)
$$

where $\Psi_{0}$ is the amplitude of the incident wave, $\vec{k}_{i}$ is the incident wave vector, and $\vec{r}$ is a space vector. If scattering takes place at a two-dimensional periodic lattice and multiple scattering is neglected, the diffracted beam can be written as [Hov86]

$$
\Psi_{s}=\Psi_{0}\left[\sum_{n} \alpha f_{n}(\vec{s}) \exp \left(\overrightarrow{i s} \cdot \overrightarrow{r_{n}}\right)\right] \exp \left(\overrightarrow{i k_{f}} \cdot \vec{r}\right)
$$

where $f_{n}(\vec{s})$ is the atomic scattering factor for the $\mathrm{n}^{\text {th }}$ atom located at position $\overrightarrow{r_{n}}, \vec{s}=\overrightarrow{k_{f}}-\overrightarrow{k_{i}}$ is the momentum transfer, $\alpha$ is a constant and $\overrightarrow{k_{f}}$ is the scattered wave vector. Here $\overrightarrow{r_{n}}$ can be replaced by $m_{1} \overrightarrow{a_{1}}+m_{2} \overrightarrow{a_{2}}$, so the diffraction intensity is

$$
I \propto \mid \sum_{m_{1} m_{2}} \exp \left[\left.\overrightarrow{i s} \cdot\left(m_{1} \overrightarrow{a_{1}}+m_{2} \overrightarrow{a_{2}}\right)\right|^{2} .\right.
$$

The maximum condition should fulfill

$$
\vec{s} \cdot\left(m_{1} \overrightarrow{a_{1}}+m_{2} \overrightarrow{a_{2}}\right)=2 n \pi \text {. }
$$

Usually when incident electrons fit this condition, they are called in-phase.

This condition can also be expressed in reciprocal space. In Eq.(2-30) the sum over the lattice vectors is proportional to the Dirac delta function $\delta\left(\overrightarrow{s_{/ /}}-\vec{g}\right)$, where $\overrightarrow{s_{/ /}}$is the component of $\vec{s}$ parallel to the surface, and $\vec{g}=h \vec{a}_{1}{ }^{*}+k \vec{a}_{2}^{*}$ ( $h, k$ integers) describes any of the two-dimensional reciprocal lattice vectors of the surface lattice $\left(\overrightarrow{a_{1}}, \overrightarrow{a_{2}}\right)$ [Hov86]. So, LEED intensity maxima are to be expected if 


$$
\vec{s}_{/ /}=h \vec{a}_{1}^{*}+k \vec{a}_{2}^{*} .
$$

This is shown in Figure 2.6.

\subsection{Auger Electron Spectroscopy}

Auger electron spectroscopy (AES) is a surface sensitive technique utilizing the emission of low energy electrons in the Auger process. AES is one of the most commonly applied analytical surface techniques for determining the composition of surface layers.
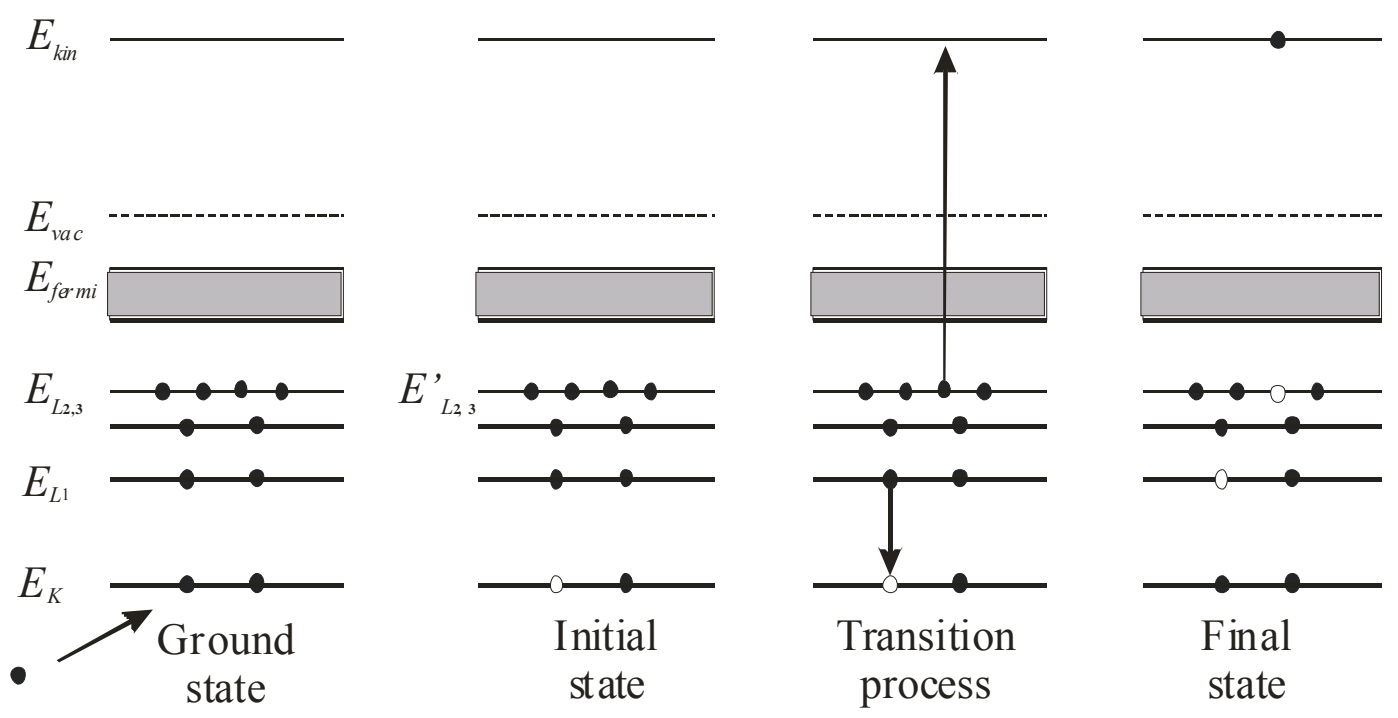

Figure 2.7 Schematic representation of the KLL Auger process in a solid. The atom in the ground state is excited by electron impact. This core hole leads to a contraction of the outer shells, denoted by $E_{L 2,3}{ }^{\prime}$. The $K$ hole is filled by an L electron in the transition process and the excess energy is transferred to another L electron which is ejected from the atom. The final state is a doubly ionized atom [Lin94].

In the Auger process, at first, an electron of the atomic inner shell is removed leaving a vacancy. Most commonly vacancies are created by bombarding the surface with an electron beam. The hole in the inner shell of the excited atom is filled by a second electron from a higher shell. At the same 
time energy is released and a third electron, the Auger electron, is emitted carrying the excess energy as kinetic energy in a radiationless process. The process of an excited ion decaying into a doubly charged ion by ejection of an electron is called the Auger process [Fle95]. The Auger process is schematically shown for a KLL transition in Figure 2.7 [Lin94]. Since the energy of the emitted Auger electron only depends on the energy of the three energy levels involved and since the binding energies of the elements are unique, each element has a characteristic pattern of Auger emission lines. So AES can be used to monitor the cleanliness of the sample surface.

AES identifies elemental compositions of surfaces by measuring the energies of Auger electrons. The general experimental setup consists of a primary source, a sample and an energy dispersive detector. All facilities are located inside an ultra-high vacuum system.

Furthermore, studying the change of the intensity of the Auger transitions when the film thickness during deposition is changed, AES can be used to characterize the growth mode of a film. If one assumes Frank-van der Merwe growth (layer by layer), the intensities can be described in the following way for each integer of evaporated atomic layers. The substrate intensity is expressed as

$$
I_{s}=I_{s 0} e^{-t /\left(\lambda_{1} \cos \theta\right)},
$$

and the film intensity as

$$
I_{F}=I_{F 0}\left(1-e^{-t /\left(\lambda_{2} \cos \theta\right)}\right) .
$$

Where $I_{\mathrm{s} 0}$ and $I_{\mathrm{F} 0}$ are the Auger intensity of the pure substrate and film, respectively, $t$ is the thickness of the film and $\theta$ is the angle of emission of the Auger electron with respect to the surface normal. $\lambda_{\mathrm{i}}(i=1,2)$ is the mean free path of the Auger electrons which is a function of the particular Auger energy [Ert85, Naw98]. 


\section{Chapter 3 Sample Preparation}

A common technique for the preparation of ultrathin magnetic metal films is Molecular Beam Epitaxy (MBE) [Art79, Cho79, Pan80]. Using MBE allows one to prepare thin crystalline films on crystalline substrates under ultrahigh vacuum (UHV) conditions. The growth process, i.e. cleanliness and crystal structure of the sample surface can be controlled using different insitu analysis techniques, such as AES and LEED.

\subsection{Basic models and principles of $\mathrm{MBE}$}

Using the MBE technique to prepare thin films, some basic models and principles turned out to be very useful in predicting and understanding epitaxial growth modes and film structures.

\section{Surface energies and equilibrium growth modes}

Bauer [Bau58] firstly noted that growth modes of thin films could be considered as a wetting problem, which was determined by

$$
\Delta r=r_{f}+r_{i}-r_{s},
$$

where $r_{s}, r_{f}$ and $r_{i}$ are the surface energies of the substrate, the growing film and the interface energy, respectively. If the film grows near equilibrium, i.e. when $\Delta r<0$, it wets the surface and therefore starts growing by a monolayer; when $\Delta r>0$ it starts growing by three-dimensional nuclei (Volmer-Weber mode of growth, island mode). For the case of stable monolayers each layer wets the previous one. This growth mode is called layer-by-layer (van der 
Merwe) growth. Otherwise three-dimensional islands are expected to nucleate on one or a few flat layers (Stranski-Krastanov mode). Because metallic interface energies are one order of magnitude lower than surface energies, the growth mode is governed basically by surface energies.

\section{Supersaturation and forced layer growth}

Because of the high supersaturation of the condensing atomic beam, the real growth process is determined to a large extent by kinetic principles. For epitaxial and polycrystalline films, generally, condensation at low temperature results in small grain sizes while condensation at high temperatures results in large grains. Accordingly, monolayer nucleation can be induced by high supersaturation (low temperature, high growth rate), resulting in a quasi van der Merwe or forced layer growth mode, which enable one to produce more or less layer by layer grown epitaxial films, even in nonwetting systems near the wetting limit.

\section{Misfit and misfit dislocations}

The lattice misfit $f$ is a very important parameter to determine epitaxial growth. In a one-dimensional model it is defined by

$$
f=\frac{(b-a)}{a} \text {, }
$$

where $b$ and a are the lattice parameters of films and substrates, respectively. If the contacting lattice planes of both crystals show the same twodimensional symmetry (homosymmetric interfaces), this definition also applies for interface. In the growing film the misfit to the substrate can be accommodated by elastic strain or by misfit dislocations [Fra49]. For small misfit, it may be energetically favourable for the film to accommodate completely to the substrate by elastic strain, forming a pseudomorphic film without any dislocations. There is a critical misfit, below which the monolayer is pseudomorphic. In general its order is about $10 \%$. For $f<0$ the film is under expansive strain and the critical misfit becomes larger. While for $f>0$ the film is under compressive strain and the critical misfit becomes smaller. 


\subsection{Experimental setup of the MBE chamber}

In this work two MBE chambers were used to prepare the samples. A stationary chamber was used to prepare the samples for the ex-situ PNR, XRR and in-situ MOKE measurements. A movable chamber was used to prepare the samples for the in-situ PNR measurements.

\section{Setup of the stationary MBE chamber}

A sketch of the stationary MBE is shown in Figure 3.1.

The chamber is pumped in series with two turbo-molecular pumps, which are pre-pumped by a rotary pump. Additionally an ion getter pump, a titanium sublimation pump and a liquid nitrogen cold trap are used, in order to achieve an ultrahigh vacuum of the order of $10^{-10}$ mbar. The residual gas in the UHV chamber can be analyzed using a quadrupole mass spectrometer.

A substrate is mounted on the sample holder that is connected to the manipulator. The latter can be controlled by a computer. The position of the sample can be changed in $\mathrm{x}, \mathrm{y}, \mathrm{z}$ directions and rotated around the axis of the manipulator. So the sample can be moved at any position and faced to the desired direction. In addition, the sample holder is equipped with a resistance heater consisting of a graphite layer which is embedded in boron nitride. In this way temperatures up to $1250 \mathrm{~K}$ can be achieved. The sample temperature is measured using a chromel-alumel thermocouple.

The electron beam evaporator is equipped with four crucibles, i.e. four different materials can be evaporated without opening the chamber. An electron beam is generated, deflected and focused by appropriate magnetic fields onto the source materials inside the crucibles. The width and flux of the electron beam can be easily adjusted to meet the requirements in order to evaporate a particular source material at a special rate. In order to avoid alloying the crucibles are cooled by flowing water. The rate of evaporation is monitored by a calibrated quartz crystal thickness monitor. A shutter can be used to cover the sample partly during the sample preparation. This is particularly useful for the preparation of wedge-shaped samples.

Furthermore, the chamber is equipped with an AES, LEED and MOKE system for the in-situ analysis of the samples. A load-lock allows one to bring out samples or to introduce new substrates into the chamber without opening 
it to air. A wobble stick helps to snatch the sample or to change its position between the transfer rod and the manipulator. In addition, a sputtering gun can be used in order to clean the substrates and samples.

\section{Setup of the movable MBE chamber}

A sketch of the movable MBE chamber is shown in Figure 5.8.

This UHV preparation chamber was specially designed and built by ourselves for the in-situ PNR measurements. Two design features had to be met for the movable MBE chamber. The first, it should have all functions of the stationary MBE chamber to prepare a sample; the second, it should be easy to be moved from the laboratory to the neutron guide hall and fit into the spare space at the reflectometer V6. The neutron beam could hit the sample which is kept under UHV conditions passing through a glass cylinder mounted at the UHV chamber. Since boron absorbs neutrons this glass cylinder has no boron component and is made from quartz glass.

\subsection{Sample preparation}

\section{Preparation of the $\mathrm{V}(\mathbf{1 0 0})$ buffer layer}

In order to investigate the $\mathrm{V} / t_{\mathrm{Fe}} \mathrm{Fe} / \mathrm{V}(100)$ or $t_{\mathrm{Fe}} \mathrm{Fe} / \mathrm{V}(100)$ system, clean $\mathrm{V}(100)$ single crystals are required. It is well known that it is very difficult to clean Vanadium substrates [Ada81, Jen82, Naw98]. Therefore, instead of using $\mathrm{V}(100)$ substrates, a clean $\mathrm{V}$ buffer layer was prepared on a $\mathrm{MgO}(100)$ substrate by molecular beam epitaxy evaporating $\mathrm{V}$ from high purity Vanadium rods. The size of the $\mathrm{MgO}(100)$ substrate is $30 \times 15 \mathrm{~mm}^{2}$.

At first a pre-cleaned $\mathrm{MgO}(100)$ substrate was introduced into the chamber. It was annealed at $900 \mathrm{~K}$ for 20 minutes to remove possible contaminations from the surface. Thereafter the cleanliness of the surface was checked by AES. If the Auger spectrum revealed that there were some contaminations left on the surface, sputtering would be carried out. Usually 25 -second sputtering with a $370 \mathrm{eV}$ argon beam were sufficient to remove the contaminations totally. After that, it was annealed at $900 \mathrm{~K}$ for 20 minutes again. Afterwards, the substrate was checked by AES and LEED. The results are shown in Figure 3.2. 


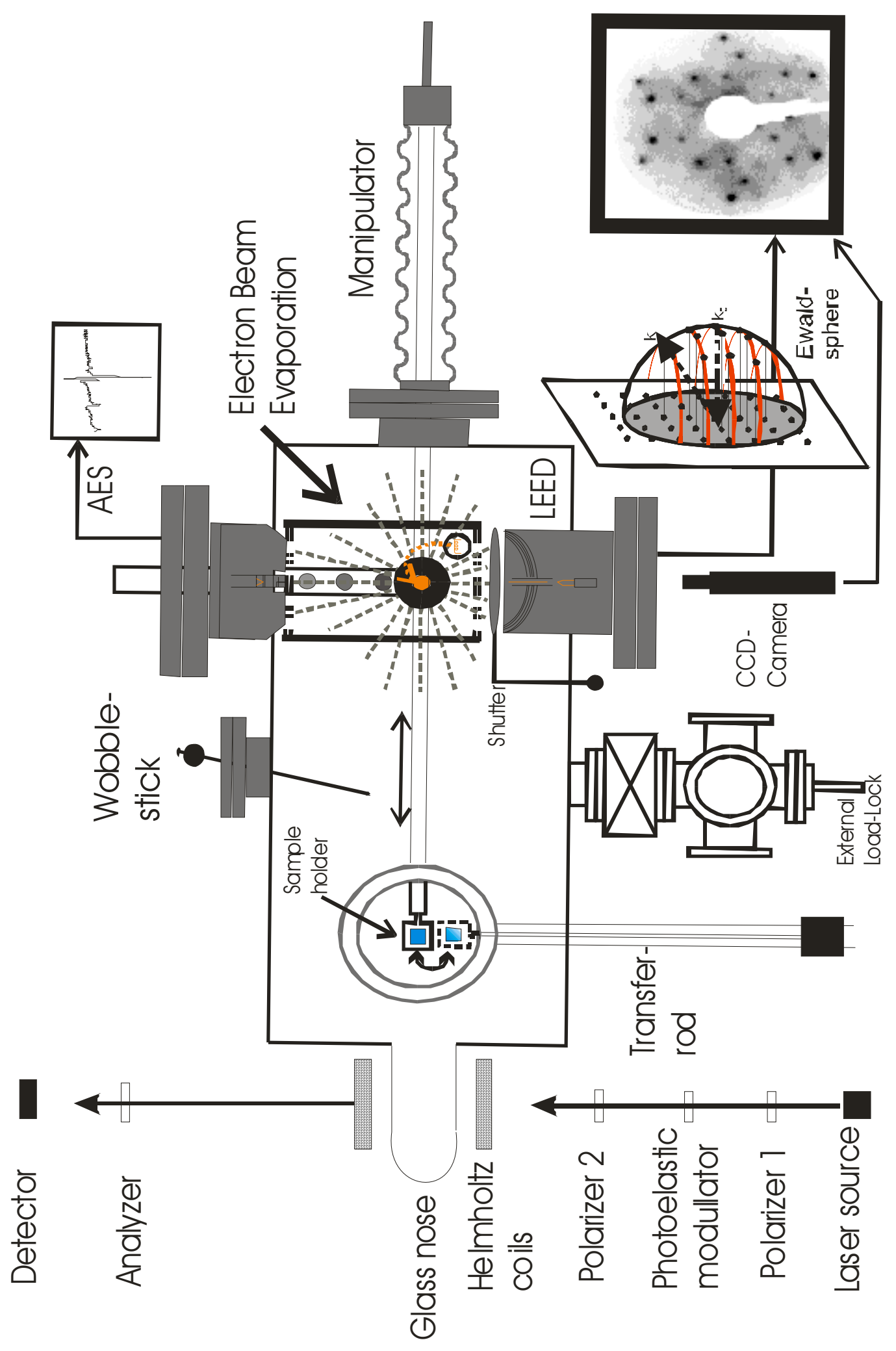

Figure 3.1 Sketch of the stationary MBE chamber 
(01)

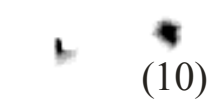

(10)

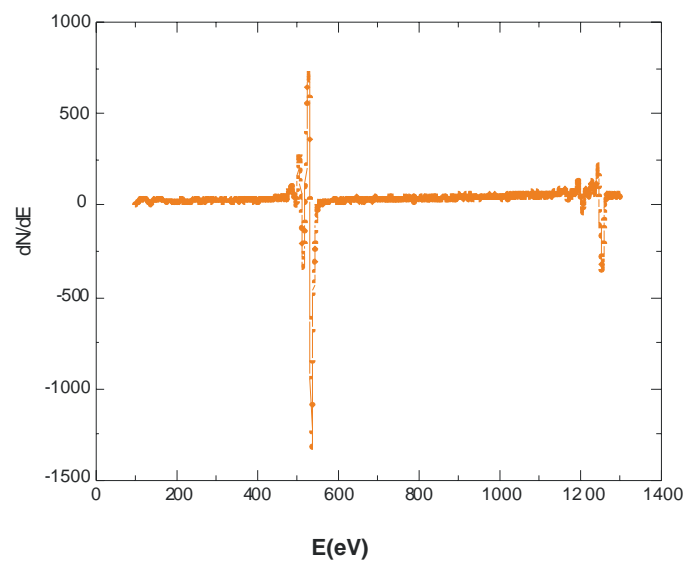

(a)

(b)

Figure 3.2 LEED pattern (a) and Auger spectrum (b) of the substrate $\mathrm{MgO}$ (100).

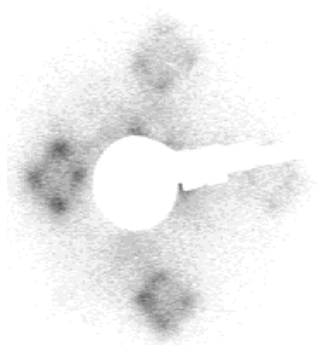

(a)

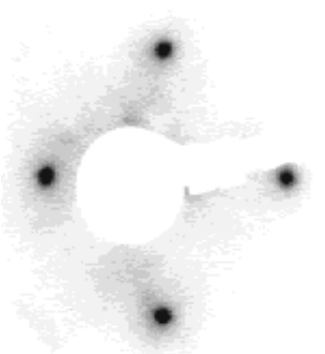

(d)

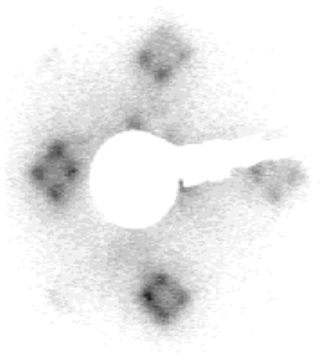

(b)

(c)

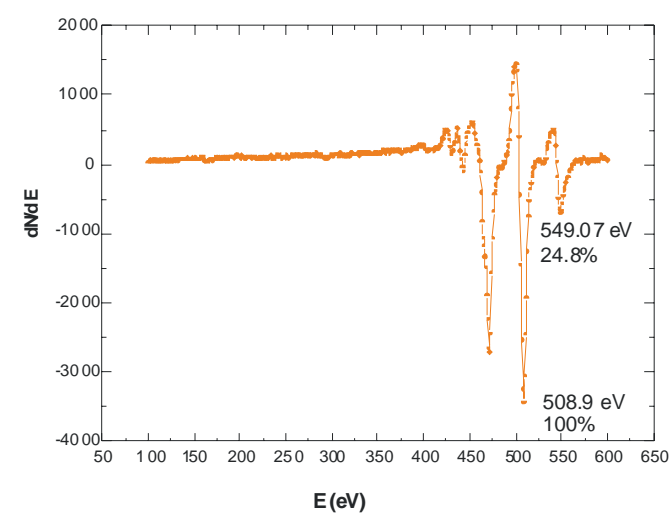

(e)

Figure 3.3 LEED patterns and Auger spectrum of the $V(100)$ grown at $300 \mathrm{~K}$. (a) $E=89.3 \mathrm{eV}$; (b) $E=94.4 \mathrm{eV}$; (c) $E=97.0 \mathrm{eV}$ (d) $E=110.7 \mathrm{eV}$ (inphase). (a), (b) and (c) are the LEED patterns where the energy is approaching to the in-phase energy as $(\mathrm{d})$. 


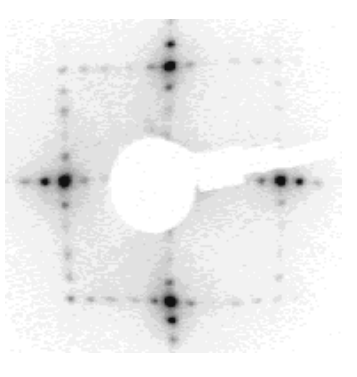

(a)

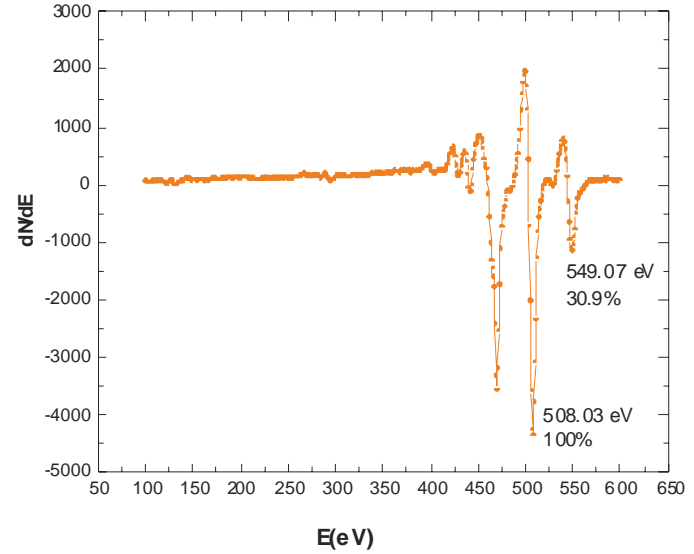

(b)

Figure 3.4 LEED pattern and Auger spectrum of the V(100) buffer layer annealed at $700 \mathrm{~K}$ for 20 minutes.

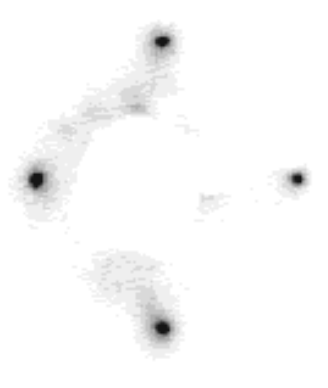

(a)

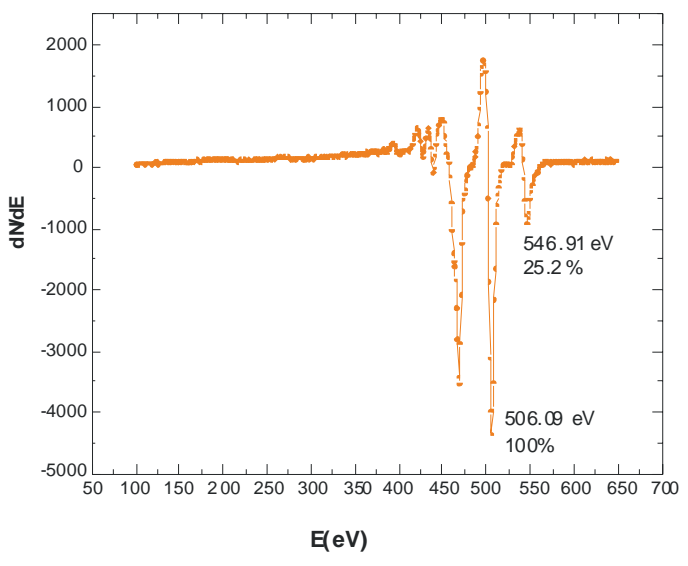

(b)

Figure 3.5 LEED pattern and Auger spectrum of the another $3 \mathrm{~nm} V$ deposited on the annealed V(100) buffer layer. 
After a clean $\mathrm{MgO}(100)$ was obtained, a V layer of $85 \mathrm{~nm}$ thickness was evaporated onto the substrate at a rate of $0.01 \mathrm{~nm} / \mathrm{s}$ at room temperature, and then annealed at $700 \mathrm{~K}$ for 20 minutes. Afterwards, another $3 \mathrm{~nm} \mathrm{~V}$ were deposited at room temperature at $0.01 \mathrm{~nm} / \mathrm{s}$. All films were checked for crystalline quality and cleanliness by LEED and Auger as shown in Figure 3.3, Figure 3.4 and Figure 3.5. It was proven, finally, that a well crystallized, clean $\mathrm{V}(100)$ buffer layer was obtained.

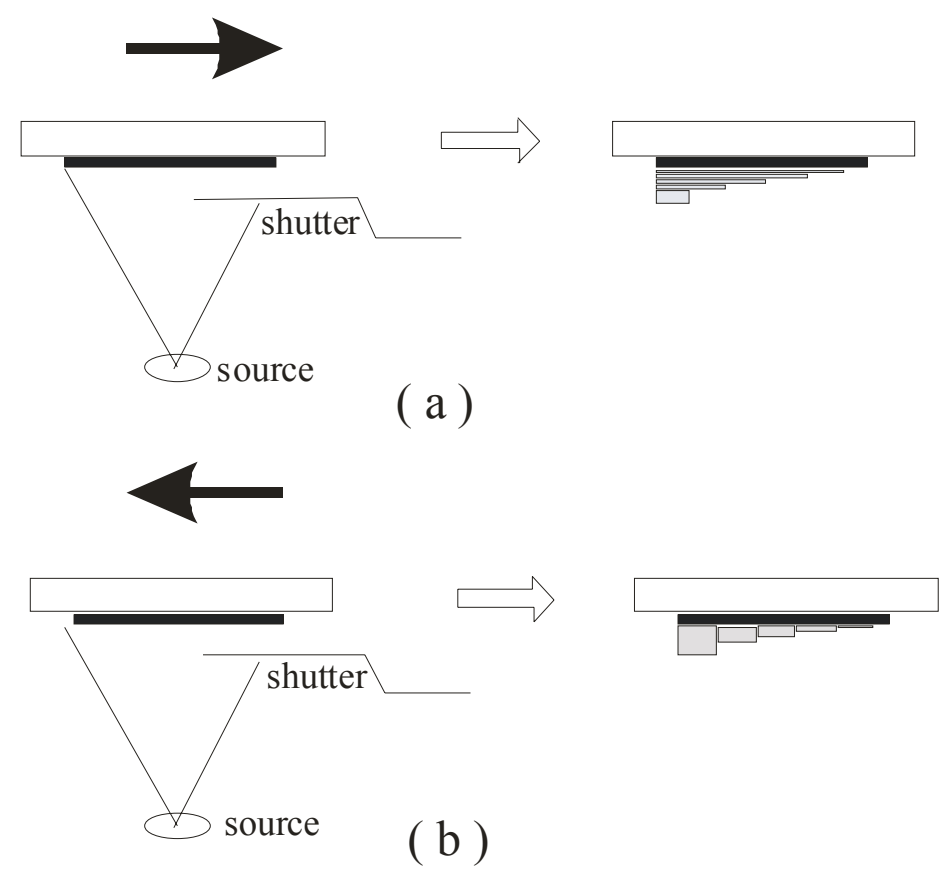

Figure 3.6 Two ways to prepare a wedge-shaped sample.

\section{Preparation of the Fe layer}

According to the requirement the different Fe layers were prepared on the clean $\mathrm{V}(100)$ buffer layers.

For the in-situ MOKE measurement a wedge-shaped Fe layer was prepared at a rate of $0.007 \mathrm{~nm} / \mathrm{s}$ in the stationary UHV chamber. As shown in Figure 3.6, there are two ways to prepare a wedge-shaped sample. The black arrows indicate the moving direction of the sample. In this work the wedgeshaped sample was prepared in the same way as shown in Figure 3.6 ( a ). 
The growth rate and thickness of the Fe layer were monitored by a calibrated quartz crystal thickness monitor. The sample position was controlled by a computer. Whenever the thickness monitor indicated an increase of the $\mathrm{Fe}$ thickness of $0.1 \mathrm{~nm}$, the sample would be moved $1 \mathrm{~mm}$ to the right as shown in Figure 3.6 ( a ). When the thickness monitor indicated that an Fe thickness of $2 \mathrm{~nm}$ was reached, the sample was moved another $1 \mathrm{~mm}$ towards the right side, and then kept at that position untill the thickness monitor indicated a thickness of $4 \mathrm{~nm}$. Thus a sample with a wedge-shaped Fe layer was prepared. Its step width is $1 \mathrm{~mm}$ and its step height is $0.1 \mathrm{~nm}$ except of the last edge at the right part of the sample. The sketch of the right part of the wedge-shaped Fe layer is shown in Figure 3.7. The thickness of the wedge-shaped Fe layer changed as $0.1 \mathrm{~nm} / \mathrm{mm}$ from $0 \mathrm{~nm}$ to $2 \mathrm{~nm}$, then the Fe layer thickness changed to $4 \mathrm{~nm}$ with a larger step height, which helped to find the starting position of the wedge and calculate the thickness of the measured part.

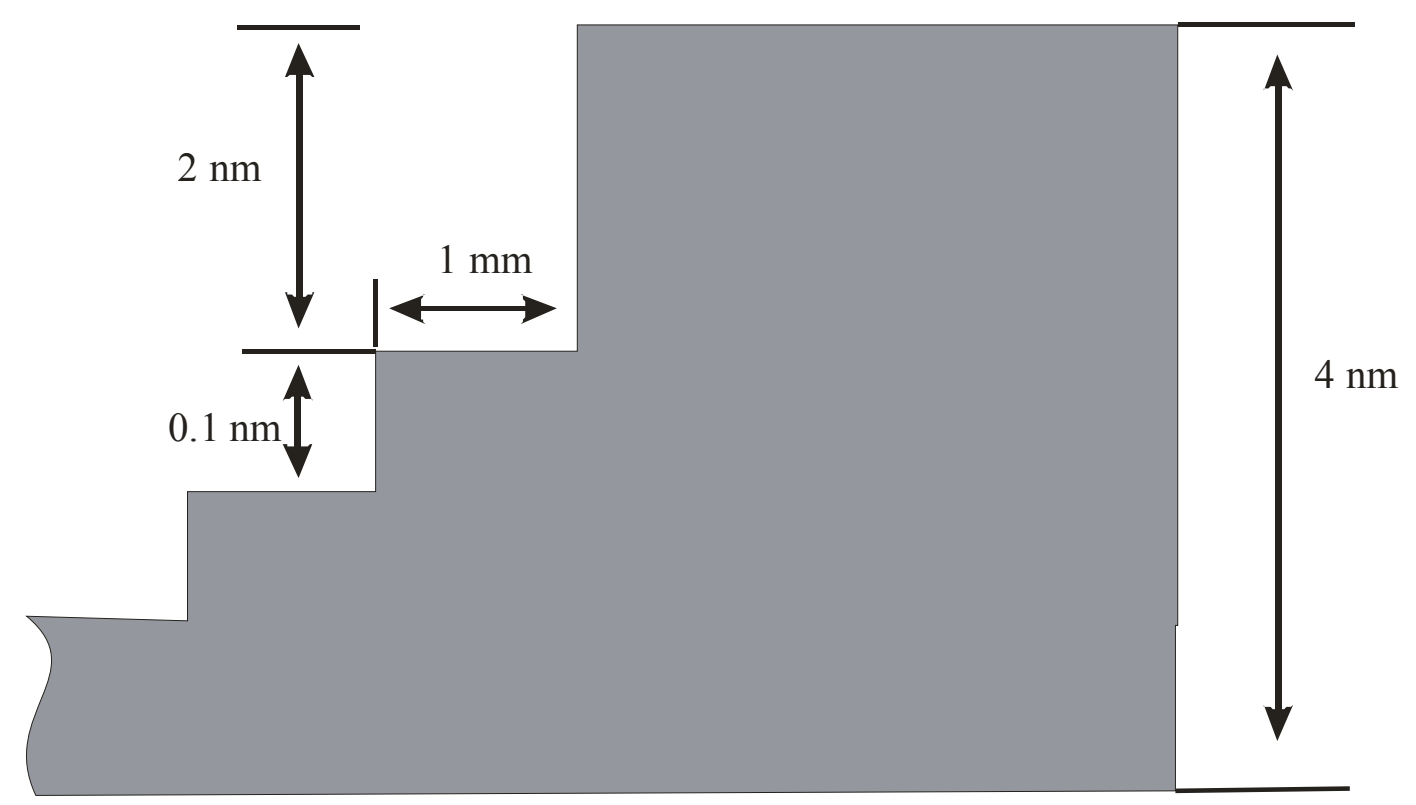

Figure 3.7 Sketch of the wedge-shaped Fe layer at the right part of the sample.

For the in-situ PNR measurements the samples with Fe layer thickness $t_{\mathrm{Fe}}$ $\left(t_{\mathrm{Fe}}=1.3,2.4,3.2,5.4 \mathrm{~nm}\right)$ were prepared. For the ex-situ PNR measurements the samples with $\mathrm{Fe}$ layer thickness $t_{\mathrm{Fe}}\left(t_{\mathrm{Fe}}=0.3,1,1.5,2,3 \mathrm{~nm}\right)$ were prepared, and $10 \mathrm{~nm} \mathrm{~V}$ capping layers were evaporated on top of the Fe in order 
to avoid $\mathrm{Fe}$ oxidation. The growth rate of the $\mathrm{Fe}$ is $0.007 \mathrm{~nm} / \mathrm{s}$. All Fe films were checked for crystalline quality and cleanliness by LEED and AES. The LEED and Auger spectrum of a sample with $t_{\mathrm{Fe}}\left(t_{\mathrm{Fe}}=1 \mathrm{~nm}\right)$ are shown in Figure 3.8.

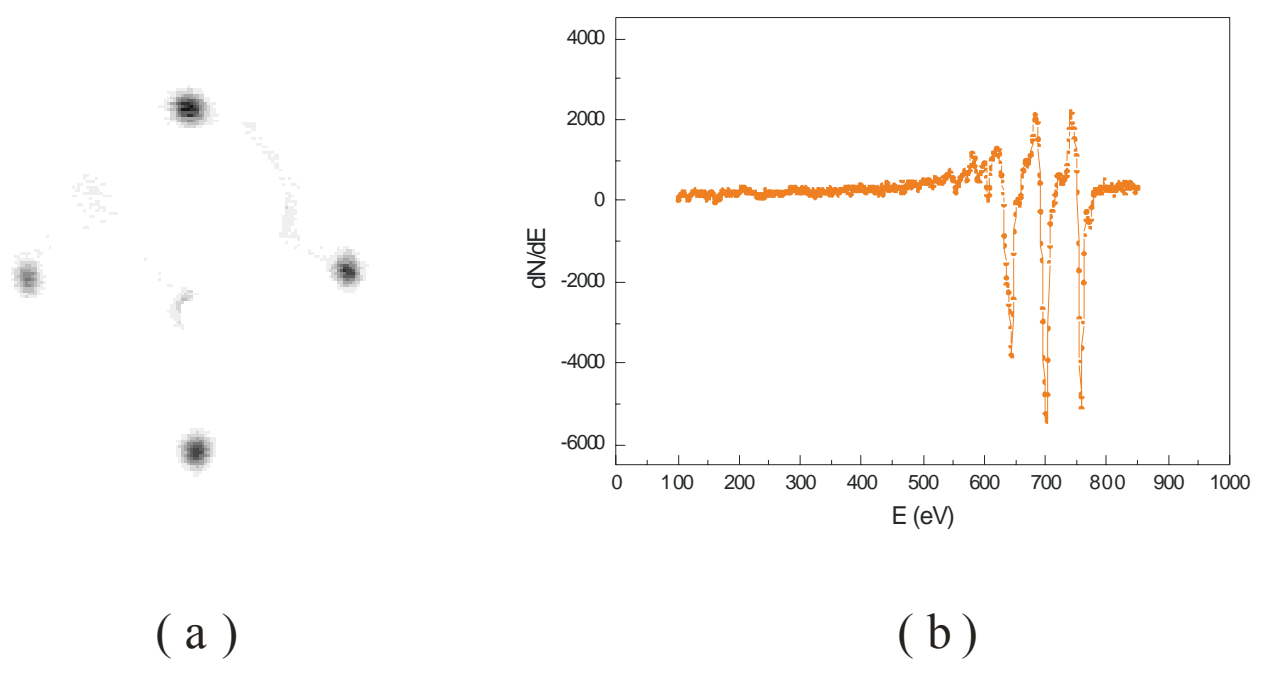

Figure 3.8 LEED pattern (a) and Auger spectrum (b) of the $1 \mathrm{~nm}$ Fe layer.

\subsection{Discussion}

The main contamination in $\mathrm{V}$ is oxygen. Usually it is very difficult to detect how much oxygen contaminates the vanadium surface. This is due to the main Auger peak of oxygen at $514 \mathrm{eV}$ which hardly can be resolved from the vanadium peak at $509 \mathrm{eV}$ other than for the case of the sulfur and carbon peaks. However, it was found that the content of oxygen would affect the intensity ratio of the $\mathrm{V}$ peak at $509 \mathrm{eV}$ relative to the main $\mathrm{V}$ peak at $473 \mathrm{eV}$ [Ada81]. If the ratio is about $25 \%$ the vanadium surface is considered to be oxygen free. The ratio increases with the content of oxygen on the surface.

The main difficulty in preparing the samples was the preparation of a $\mathrm{V}(100)$ buffer layer. Two requirements had to be met: 1 . The surface of the $\mathrm{V}(100)$ buffer layer should be clean enough in order not to affect the magnetism of the following Fe layer; 2. The V(100) buffer layer should have a good crystal quality. Admittedly it is very difficult to realize these two aspects at 
the same time because it was found that the oxygen would diffuse to the surface from the bulk when the V(100) buffer layer was annealed to improve its crystalline quality.

In the works of [Dul01, Foo83, Kre00, Tur01] it was stated that the main contamination in $\mathrm{V}$ was oxygen due to the outgassing of the $\mathrm{V}$ target. The oxygen concentration on the surface of the $\mathrm{V}$ film could be controlled by varying the $\mathrm{V}$ growth rate. For small growth rates, the oxygen concentration could be omitted at the surface if the $\mathrm{V}$ layer was prepared at a temperature below $500 \mathrm{~K}$. The oxygen segregation was limited below that temperature. In order to minimize the concentration of the oxygen in the $\mathrm{V}$ buffer layer, in this work a $85 \mathrm{~nm} V$ layer was deposited on the $\mathrm{MgO}(100)$ substrate at a very slow rate of $0.01 \mathrm{~nm} / \mathrm{s}$ at room temperature. In its Auger spectrum (see Figure 3.3) the intensity ratio of the $\mathrm{V}$ peaks at $509 \mathrm{eV}$ and $473 \mathrm{eV}$ was $24.8 \%$, i.e. the surface of the $\mathrm{V}$ layer was oxygen free after preparation at room temperature (owing to the charging the characteristic energy values are shifted with respect to the theoretical values). But the LEED patterns as seen in Figure 3.3 (a), (b) and (c), where the energy $E$ of the primary electrons was lower than the in-phase energy of $110.7 \mathrm{eV}$ in Figure 3.3 (d), showed an energy dependent splitting. The $\{10\}$ reflection of the (100) surface splitted into four spots along the [100] and [010] direction. This kind of phenomenon revealed that the (100) oriented surface was faceted as mentioned in [Alb93, Bes75, Hen70, Rhe77]. Similar patterns as a function of energy were also observed by Nawrath et al. [Naw98] for the case of a V(110) surface, but the splitting was seen only along the [1 10$]$ direction. This difference is attributed to different symmetries of the (100) and (110) surfaces.

In order to improve its crystalline quality, the $85 \mathrm{~nm} \mathrm{~V}$ buffer was annealed at $700 \mathrm{~K}$ for 20 minutes, which was proven to be the most effective among many different annealing procedures. But after this annealing treatment the LEED pattern changed from a $(1 \times 1)$ structure to a $(5 \times 5)$ superstructure and the intensity ratio of the $\mathrm{V}$ peaks at $509 \mathrm{eV}$ and $473 \mathrm{eV}$ in the Auger spectra increased from $24.8 \%$ to $30.9 \%$ as shown in Figure 3.4. That means due to the annealing the oxygen diffused to the surface and induced this superstructure. In [Tur01] the $(5 \times 5)$ reconstructed surface was studied using scanning tunneling microscopy (STM) facilities. The STM images showed that the surface preferred to develop $(5 \times 1)$ and $(1 \times 5)$ randomly distributed structures rather than only one $(5 \times 5)$ structure. 
Since oxygen diffused to the surface only after annealing, another $3 \mathrm{~nm}$ of $\mathrm{V}$ were deposited at a rate of $0.01 \mathrm{~nm} / \mathrm{s}$ at room temperature. The results of the LEED and AES analysis are shown in Figure 3.5, indicating that the (100) oriented $\mathrm{V}$ surface finally had an excellent crystalline quality (which was also proved later from the rocking scan of the ( 200 ) peak of the $\mathrm{V}$ and $\mathrm{Fe}$ layer using x-ray diffractometry, see Figure 4.8, Figure 4.9) and was oxygen free (the intensity ratio: $25.2 \%$ ) at the same time.

After a clean $\mathrm{V}(100)$ buffer layer was obtained, the Fe layer was prepared on it. The lattice constant of the $\mathrm{Fe}$ is $0.2866 \mathrm{~nm}$ and the lattice constant of the $\mathrm{V}$ is $0.303 \mathrm{~nm}$. According to Eq. (3-2) the misfit $f$ is about $-5.6 \%$. This value is smaller than the critical misfit of $10 \%$. In addition $\mathrm{Fe}$ and $\mathrm{V}$ have similar crystallographic structures (bcc). Therefore it is easy to produce a good epitaxial Fe layer on the $\mathrm{V}(100)$ buffer layer, as can be seen from the sharp LEED pattern in Figure 3.8.

In Ref [Chr95, Gra98, Pou97] it was mentioned that the in-plane orientation of the $\mathrm{V}$ film was rotated by $45^{\circ}$ with respect to the $\mathrm{MgO}(100)$ substrate. But comparing of the LEED pattern of $\mathrm{MgO}(100)$ substrate in Figure 3.2 with the LEED pattern of the V(100) buffer layer in Figure 3.3, Figure 3.4, and Figure 3.5, this kind of rotation could not be observed here. The reason might be due to the different sample preparation conditions. In Ref [Chr95, Gra98, Pou97] the samples were grown at $600 \mathrm{~K}$. But in this work the V buffer layers were grown at room temperature, and then annealed at $700 \mathrm{~K}$ for 20 minutes. 


\section{Chapter 4 The $V / t_{\mathrm{Fe}} \mathrm{Fe} / \mathrm{V}(100)$ system}

\subsection{Measurements at room temperature}

\subsubsection{Motivation}

The samples $\mathrm{V} / t_{\mathrm{Fe}} \mathrm{Fe} / \mathrm{V} / \mathrm{MgO}(100)\left(t_{\mathrm{Fe}}=0.3,1,1.5,2,3 \mathrm{~nm}\right)$ were measured by ex-situ PNR and XRR at room temperature. The goal of these investigations was to separate the interface contributions from the total magnetic moment of the films.

\subsubsection{Experimental setup}

\section{Ex-situ PNR setup}

The PNR measurements were carried out at the reflectometer V6 [Mez95] at the Hahn-Meitner-Institut using polarized neutrons with a wavelength of $0.466 \mathrm{~nm}$. The standard setup of the reflectometer is shown in Figure 4.1. The samples were mounted at the center of an electromagnet which provided a horizontal magnetic field perpendicular to the plane of reflection. The magnetic field applied was $0.25 \mathrm{~T}$ and has been proven to be strong enough to saturate the samples. The beam was collimated by two sets of computer controlled cadmium slits. The angle of incidence was varied by a precise tilting of the sample surface relative to the fixed collimated neutron beam. Using a polarizing supermirror [Kri95] and a spin flipper, polarized spin-down or spin-up neutrons were obtained. A guide field was applied throughout the beam path using special constructions of permanent magnets supplying a magnetic field along the horizontal direction, perpendicular to the beam path. 
The detector was an array of ${ }^{3} \mathrm{He}$ gas detectors with an efficiency higher than $90 \%$ for a neutron wavelength of $0.4 \mathrm{~nm}$.

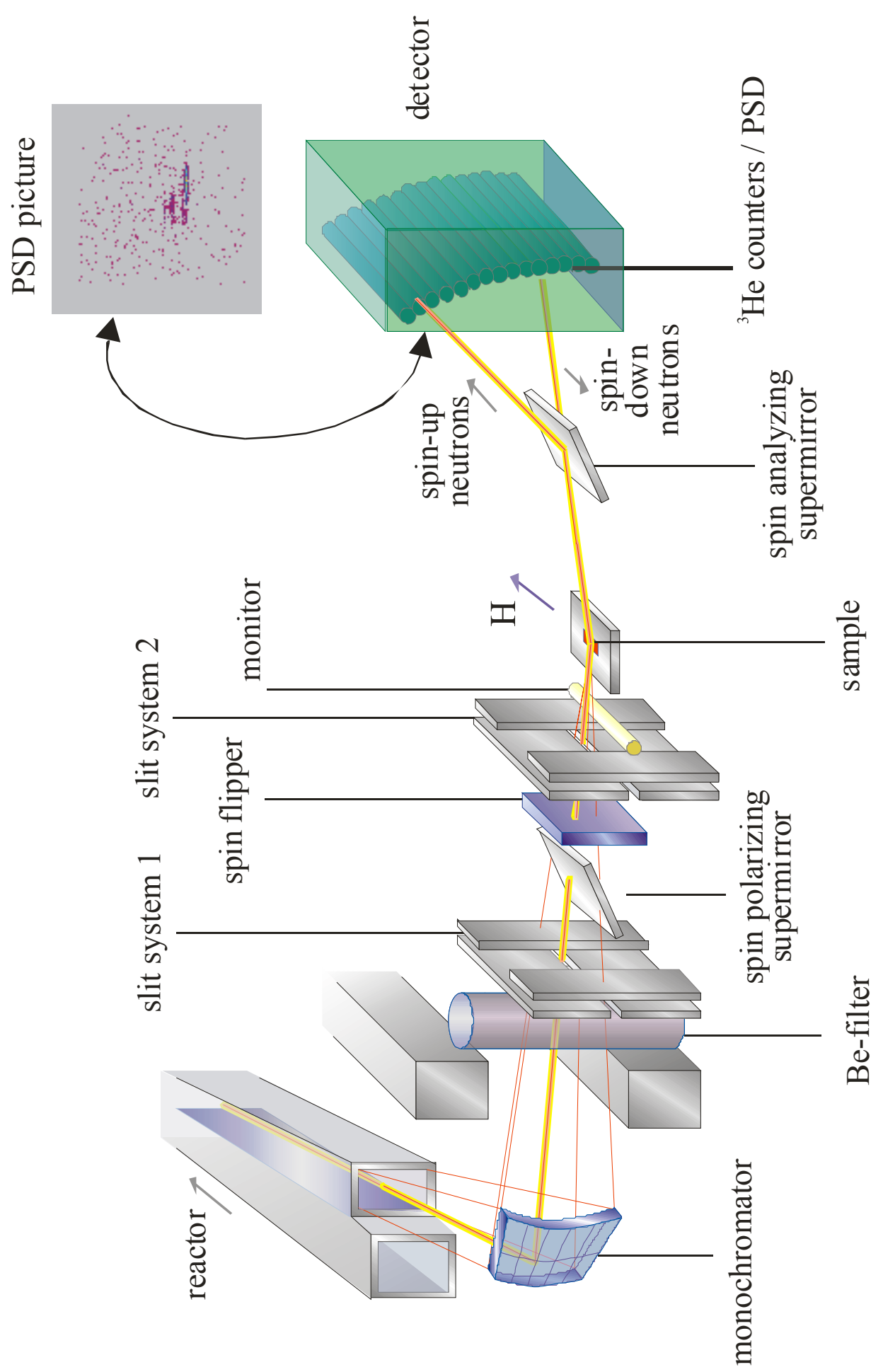

Figure 4.1 Standard setup of the reflectometer V6 at the Hahn-MeitnerInstitut 


\section{Ex-situ XRR setup}

The XRR measurements were performed at the Hahn-Meitner-Institut. The schematic drawing of the XRR is shown in Figure 4.2. The primary beam of this instrument was defined by the line focus of a sealed $\mathrm{x}$-ray tube $(0.04 \mathrm{~mm}$ $\times 8 \mathrm{~mm}, \mathrm{Cu}$ anode $)$ and a diaphragm $(0.2 \mathrm{~mm} \times 8 \mathrm{~mm})$ at a distance of 500 $\mathrm{mm}$. The collimating slits in front of and behind the sample could be adjusted manually. The reflected beam was monochromatized by a pyrolytic graphite crystal and counted by the pulse height discriminator of the scintillation detector. The resolution of this instrument was about $0.03 \mathrm{~nm}^{-1}$. The graphite crystal was set to reflect the $\mathrm{Cu}-\mathrm{K} \alpha$ doublet $(0.1541 \mathrm{~nm})$. If the intensity was very high, such as for the direct beam or in the region of total reflection, a remote controlled Ni-absorber was inserted in front of the detector in order to prevent overloading [How01]. Comparing the intensity of the neutron source with that of the x-ray source, the latter was much higher. The maximum $q$ value $\left(q_{\max }=4.94 \mathrm{~nm}^{-1}\right)$ that was reached in the XRR measurements was much larger than that $\left(q_{\max }=0.73 \mathrm{~nm}^{-1}\right)$ in the PNR measurements.

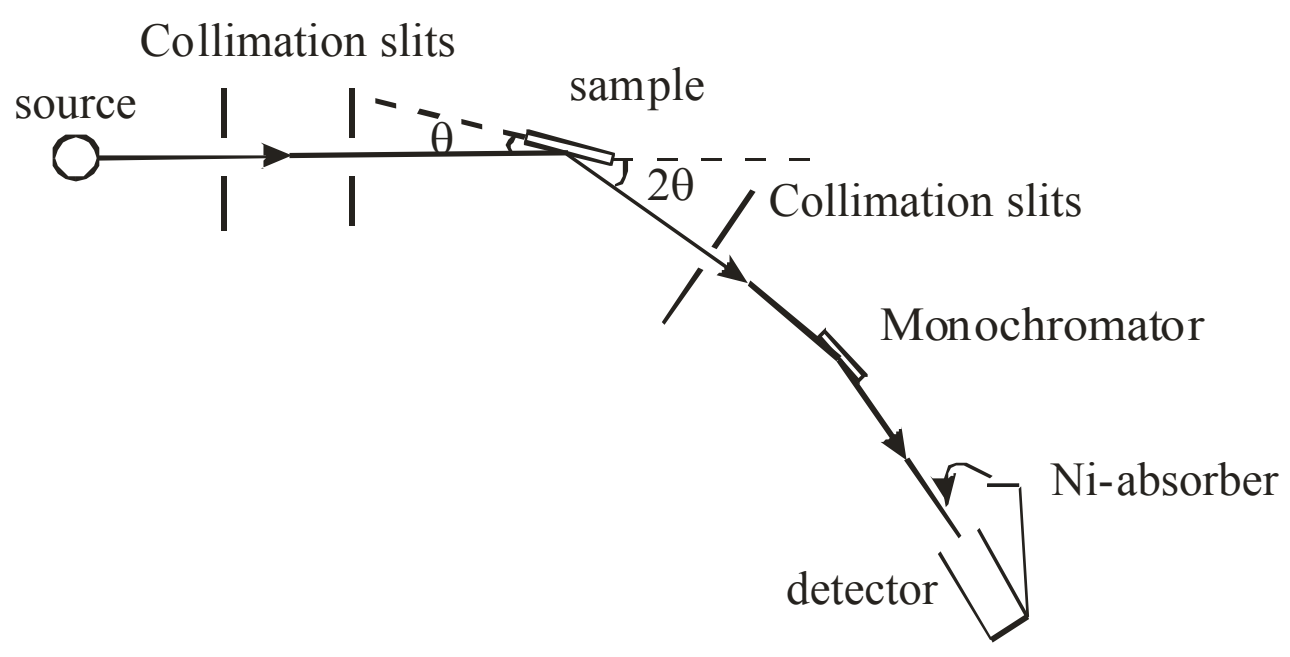

Figure 4.2 Schematic drawing of the x-ray reflectometer setup. 


\subsubsection{Experimental results}

The reflectivity curves of the samples obtained from the ex-situ PNR measurements and the corresponding spin asymmetry curves are shown in Figure 4.3, Figure 4.4 and Figure 4.5. The reflectivity curves of the samples obtained from the XRR measurements are shown in Figure 4.6 and Figure 4.7. In these pictures the reflectivity and spin asymmetry curves are shown as a function of the scattering vector $q$ whose value is defined as $q=4 \pi \sin \theta / \lambda$, where $\theta$ is the angle of incidence and $\lambda$ is the neutron/x-ray wavelength. For the present investigations it turned out that $\mathrm{x}$-rays were more sensitive to oxidations of the Vanadium and surface roughnesses than neutrons. This is due to the different scattering lengths of the oxidations of the Vandadium for neutrons and $\mathrm{x}$-rays and the larger accessible q-range in the XRR measurements, respectively. In order to get more accurate values for the thickness and roughness of the layers, the information obtained from PNR and XRR measurements were combined for the simulations. The simulations were done using a software based on the Parratt formalism [Par54, Bra]. If there is a non vanishing magnetic moment in the films, according to Eq. (2-17) and Eq. (2-19), it is known that: 1 . the reflectivity of spin-up neutrons and spin-down neutrons is different; 2 . the reflectivity difference between spin-up and spindown neutrons can be seen directly from the spin asymmetry. Herewith the thickness of the layers, roughness as well as the effective magnetic moments were determined by fitting the reflectivity of the spin-up and spin-down neutrons, the spin asymmetry curves and the reflectivity of $\mathrm{x}$-ray. The simulation results are shown as solid lines in the corresponding figures and the obtained values are given in Table 4-1.

Furthermore, the crystalline qualities of all $\mathrm{Fe}$ and $\mathrm{V}$ layers were checked by X-ray diffractometry (XRD). The rocking curves of the (200) Bragg peaks are shown in Figure 4.8 and Figure 4.9. The full-width at half-maximum (FWHM) of the $\mathrm{V}(200)$ peak is about $0.2^{\circ}$, the FWHM of the Fe(200) is about $0.05^{\circ}$.

From the results of the ex-situ PNR measurements, the products of the magnetic moment per atom and the Fe thickness were derived and plotted versus the Fe thickness $t_{\mathrm{Fe}}$ in Figure 4.10. The products are proportional to the effective magnetic moment of the Fe films. A linear fit in the region of $0.97 \mathrm{~nm} \leq t_{\mathrm{Fe}} \leq 3.03 \mathrm{~nm}$ results in a slope of $2.14( \pm 0.02) \mu_{\mathrm{B}} /$ atom and an intersection with the abscissa at $0.10 \pm(0.01) \mathrm{nm}$. 


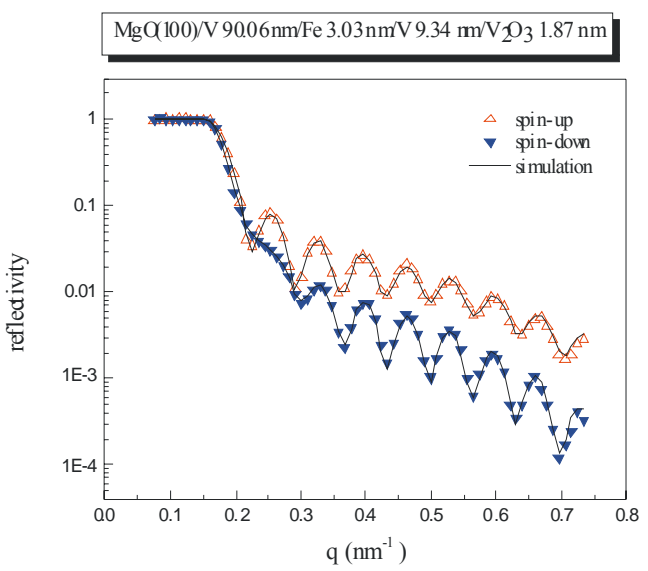

( a )

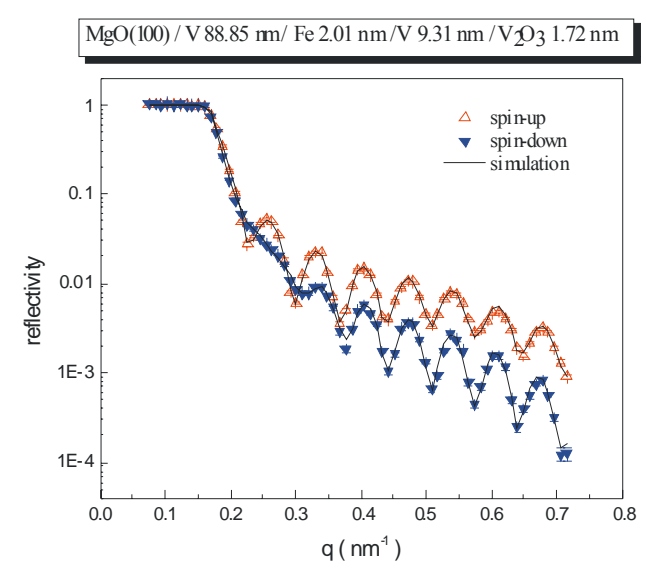

( c )

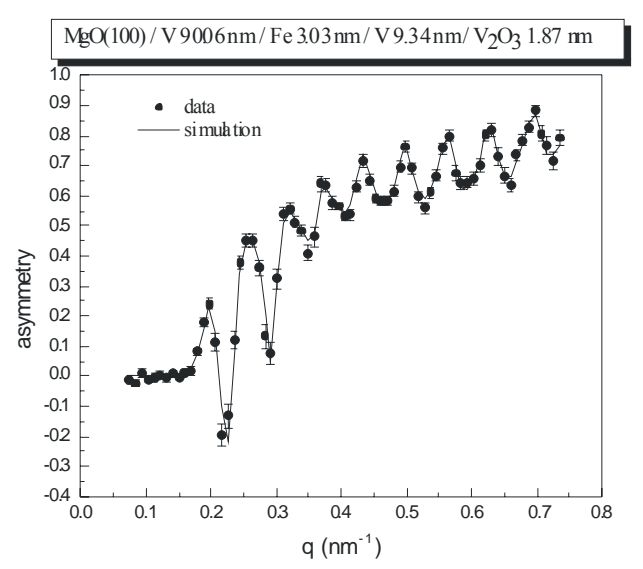

( b )

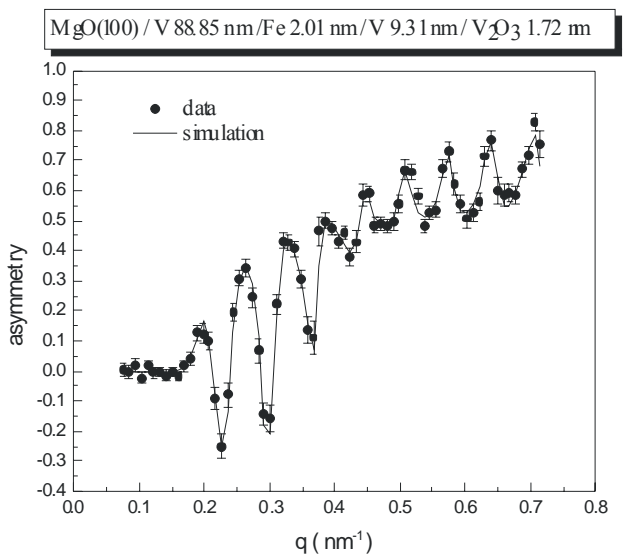

(d)

Figure 4.3 Reflectivity and spin asymmetry curves of the samples with $t_{F e}\left(t_{F e}\right.$ $=3.03 \mathrm{~nm}, 2.01 \mathrm{~nm}$ ) as obtained from ex-situ PNR measurements The reflectivity curves of the spin-up (spin-down) neutrons are given as open up-triangles ( solid down-triangles). The spin asymmetry curves are shown as dots. The solid lines are results from simulations. 


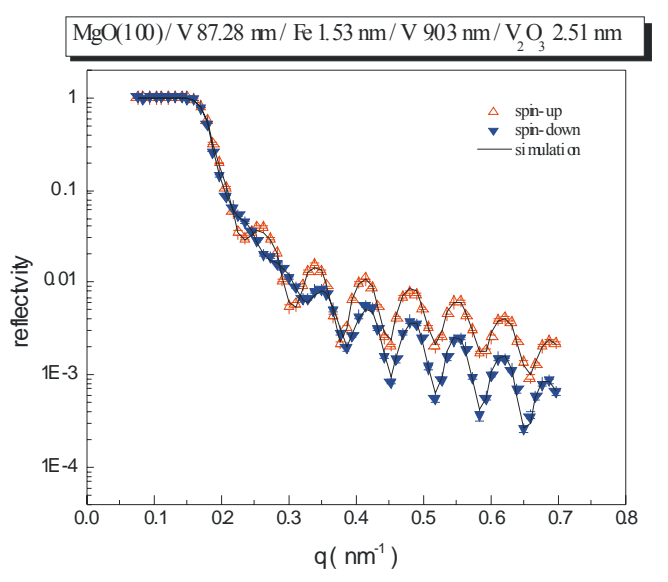

( a )

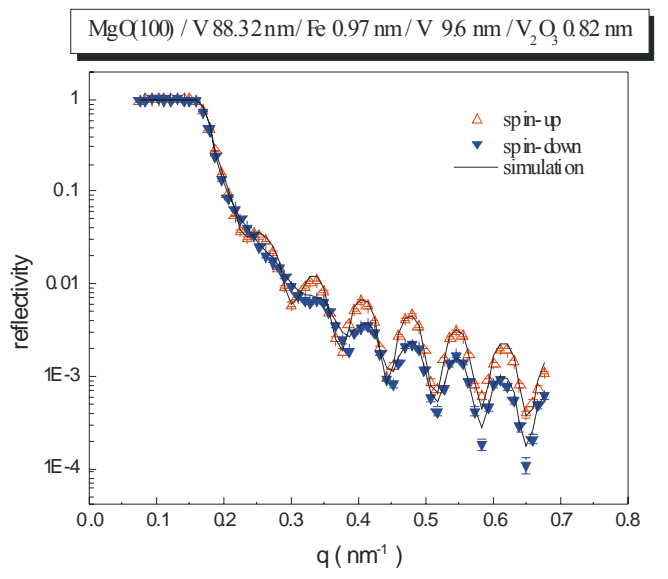

( c )

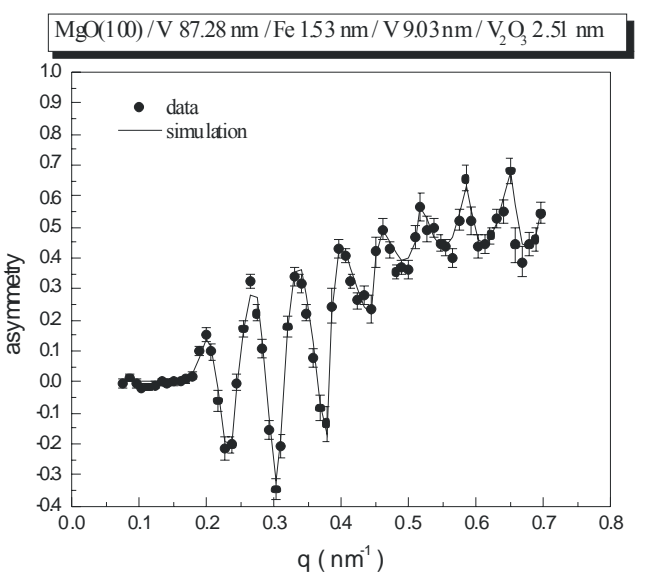

( b )

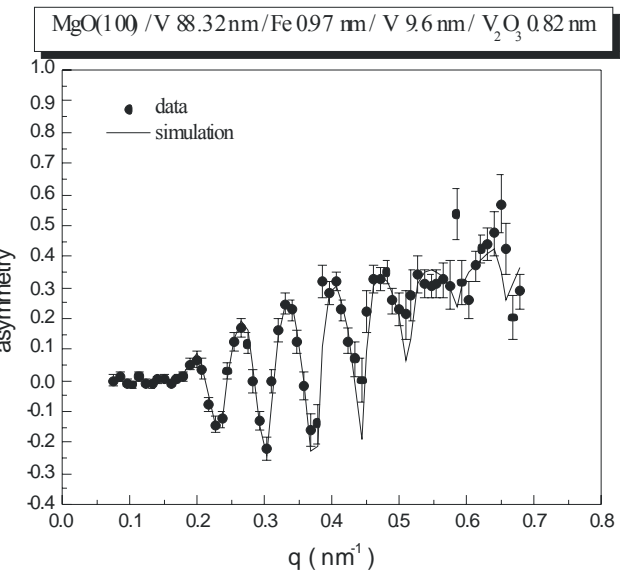

( d )

Figure 4.4 Reflectivity and spin asymmetry curves of the samples with $t_{F e}\left(t_{F e}\right.$ $=1.53 \mathrm{~nm}, 0.97 \mathrm{~nm}$ ) as obtained from ex-situ PNR measurements The reflectivity curves of the spin-up (spin-down) neutrons are given as open up-triangles ( solid down-triangles ). The spin asymmetry curves are shown as dots. The solid lines are results from simulations. 


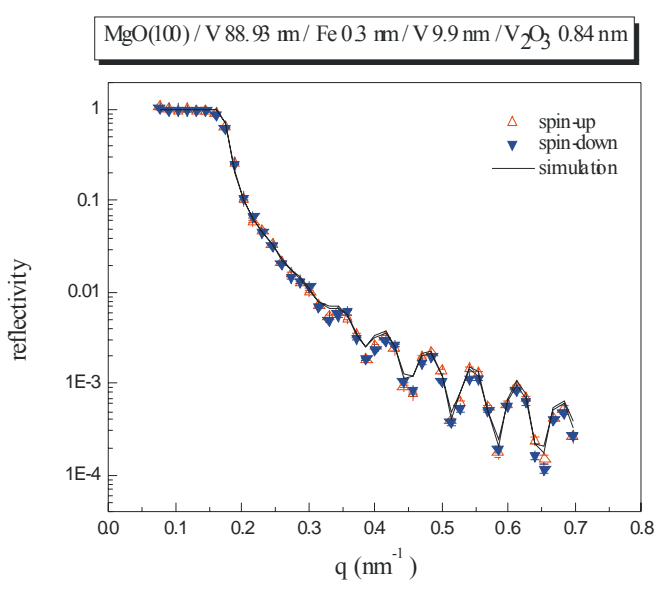

( a )

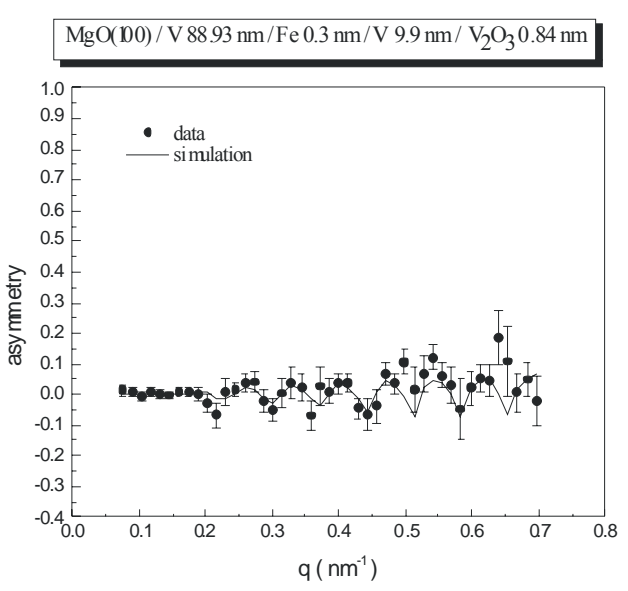

( b )

Figure 4.5 Reflectivity and spin asymmetry curves of the samples with $t_{F e}\left(t_{F e}\right.$ $=0.3 \mathrm{~nm}$ ) as obtained from ex-situ PNR measurements. The reflectivity curve of the spin-up (spin-down) neutrons is given as open up-triangles ( solid down-triangles). The spin asymmetry curve is shown as dots. The solid lines are results from simulations.

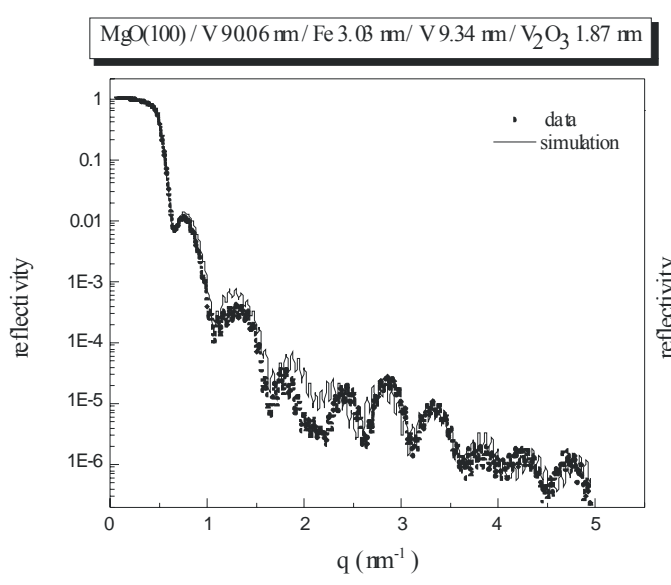

( a )

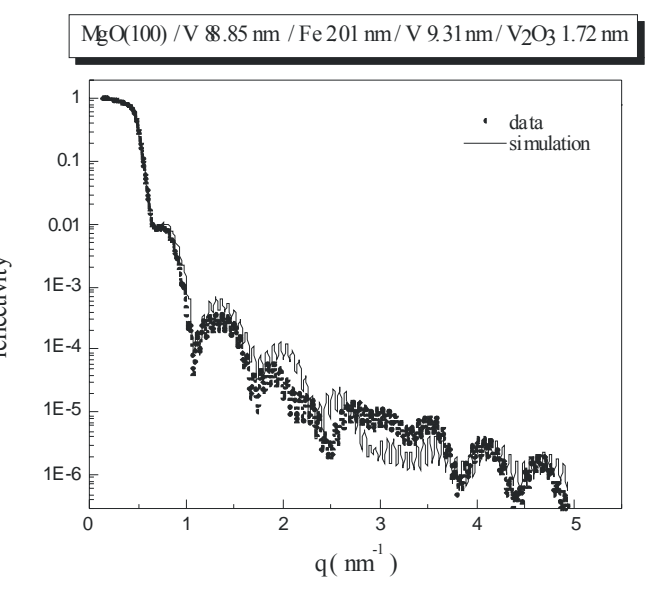

( b )

Figure 4.6 Reflectivity curves of the samples with $t_{F e}\left(t_{F e}=3.03 \mathrm{~nm}, 2.01 \mathrm{~nm}\right.$, as obtained from ex-situ XRR measurements. The data are given as dots. The solid lines are results from simulations. 


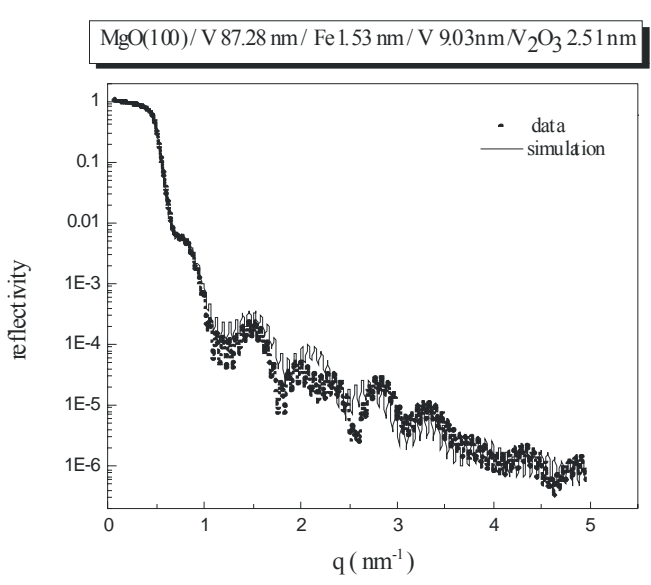

( a )

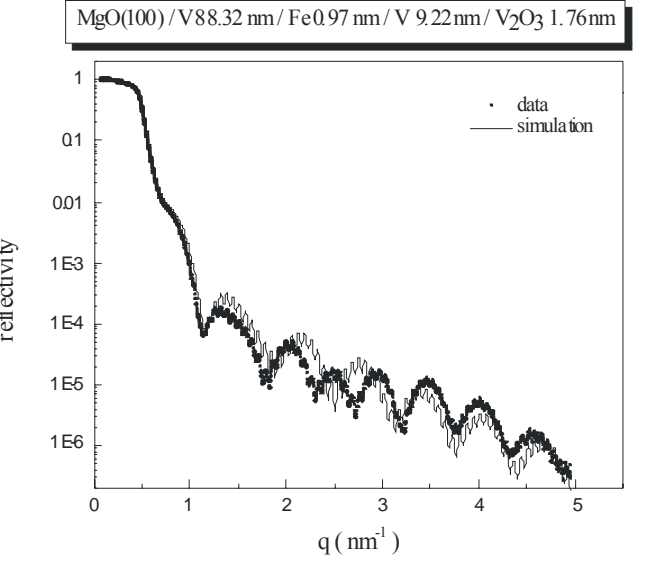

(b)

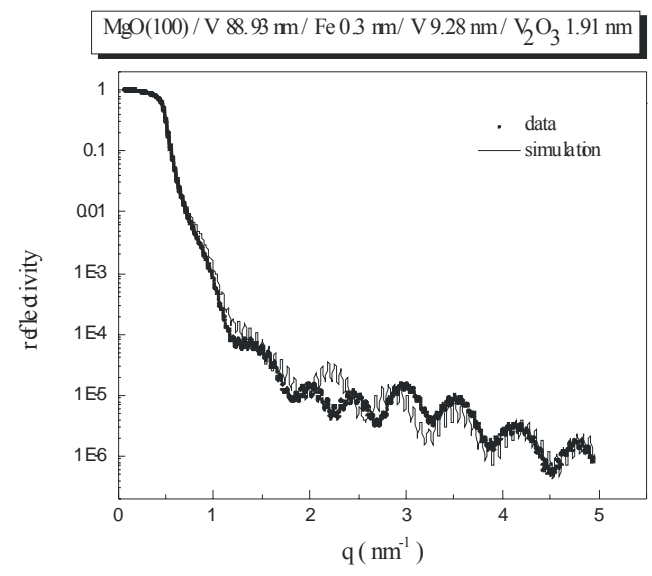

(c)

Figure 4.7 Reflectivity curves of the samples with $t_{F e}\left(t_{F e}=1.53 \mathrm{~nm}, 0.97 \mathrm{~nm}\right.$, $0.3 \mathrm{~nm}$ ) as obtained from ex-situ XRR measurements. The data are given as dots. The solid lines are results from simulations. 


\begin{tabular}{|c|c|c|c|c|c|c|c|c|c|c|c|}
\hline No. & $\begin{array}{l}\text { Measu- } \\
\text { rement }\end{array}$ & $\begin{array}{l}\sigma_{\mathrm{MgO}} \\
(\mathrm{nm})\end{array}$ & $\begin{array}{c}t_{\mathrm{V}} \\
(\mathrm{nm})\end{array}$ & $\begin{array}{c}\sigma_{\mathrm{V}} \\
(\mathrm{nm})\end{array}$ & $\begin{array}{c}t_{\mathrm{Fe}} \\
(\mathrm{nm})\end{array}$ & $\begin{array}{l}\sigma_{\mathrm{Fe}} \\
(\mathbf{n m})\end{array}$ & $\begin{array}{c}\mathbf{m}_{\mathrm{Fe}} \\
\left(\mu_{\mathrm{B}} / \text { atom }\right)\end{array}$ & $\begin{array}{c}t_{\mathrm{V}} \\
(\mathrm{nm})\end{array}$ & $\begin{array}{c}\sigma_{\mathrm{V}} \\
(\mathbf{n m})\end{array}$ & $\begin{array}{l}t_{\mathrm{V} 2 \mathrm{O}} \\
(\mathrm{nm})\end{array}$ & $\begin{array}{l}\sigma_{\mathrm{V} 203} \\
(\mathrm{~nm})\end{array}$ \\
\hline \multirow{2}{*}{1} & PNR & 0.2 & 90.06 & 0.2 & 3.03 & 0.4 & 2.07 & 9.34 & 0.2 & 1.87 & 1.6 \\
\hline & XRR & 0.2 & 90.06 & 0.2 & 3.03 & 0.4 & - & 9.34 & 0.2 & 1.87 & 1.6 \\
\hline \multirow{2}{*}{2} & PNR & 0.2 & 88.85 & 0.2 & 2.01 & 0.2 & 2.02 & 9.31 & 0.3 & 1.72 & 1.49 \\
\hline & XRR & 0.2 & 88.85 & 0.2 & 2.01 & 0.2 & 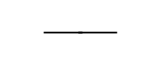 & 9.31 & 0.3 & 1.72 & 1.49 \\
\hline \multirow{2}{*}{3} & PNR & 0.1 & 87.28 & 0.1 & 1.53 & 0.4 & 1.98 & 9.03 & 0.4 & 2.51 & 1.69 \\
\hline & XRR & 0.1 & 87.28 & 0.1 & 1.53 & 0.4 & - & 9.03 & 0.4 & 2.51 & 1.69 \\
\hline \multirow{2}{*}{4} & PNR & 0.2 & 88.32 & 0.2 & 0.97 & 0.3 & 1.94 & 9.60 & 0.2 & 0.82 & 0.5 \\
\hline & XRR & 0.2 & 88.32 & 0.2 & 0.97 & 0.3 & 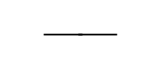 & 9.22 & 0.2 & 1.76 & 1.2 \\
\hline \multirow{2}{*}{5} & PNR & 0.1 & 88.93 & 0.1 & 0.30 & 0.2 & 0.57 & 9.90 & 0.2 & 0.84 & 0.5 \\
\hline & XRR & 0.1 & 88.93 & 0.1 & 0.30 & 0.2 & - & 9.28 & 0.2 & 1.91 & 1.14 \\
\hline
\end{tabular}

Table 4-1 Simulation results from the data of PNR and XRR measurements. $\sigma_{\mathrm{MgO},}, \sigma_{\mathrm{V}}, \sigma_{\mathrm{Fe}}$ and $\sigma_{\mathrm{V} 2 \mathrm{O} 3}$ are the surface roughnesses of the substrate $\mathrm{MgO}$, the $\mathrm{V}, \mathrm{Fe}$ and $\mathrm{V}_{2} \mathrm{O}_{3}$ layers. $t_{V} t_{\mathrm{Fe}}$ and $t_{\mathrm{V} 2 \mathrm{O}}$ are the thicknesses of the $\mathrm{V}, \mathrm{Fe}$ and $\mathrm{V}_{2} \mathrm{O}_{3}$ layers respectively. $m_{\mathrm{Fe}}$ is the magnetic moment of the Fe atom. 


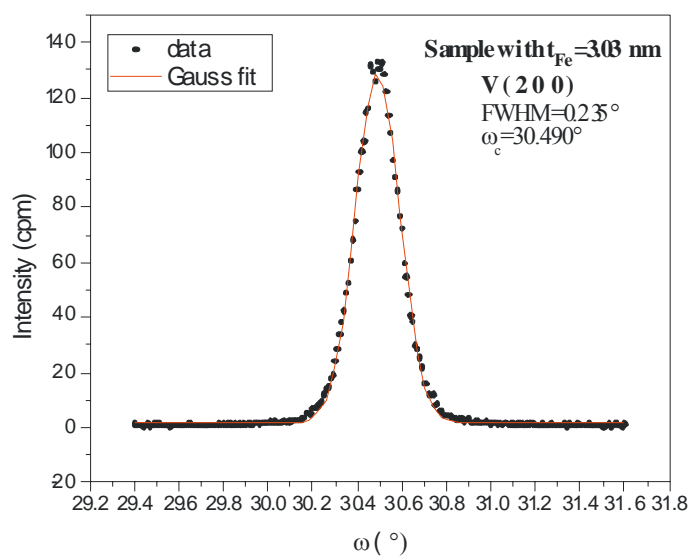

( a )

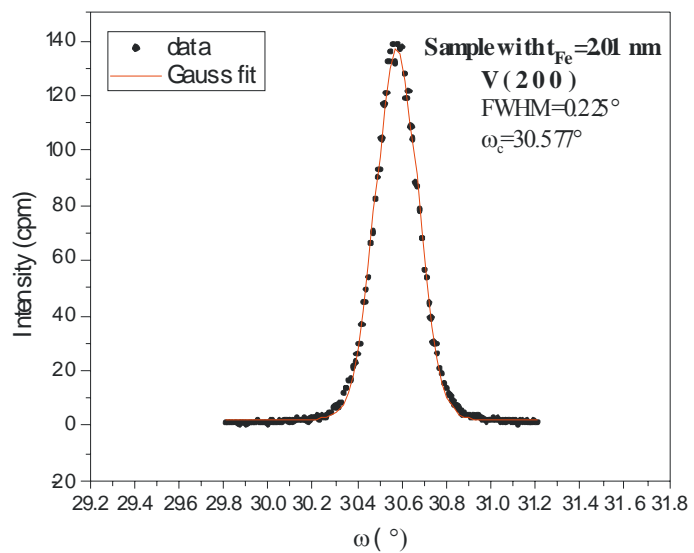

(c)

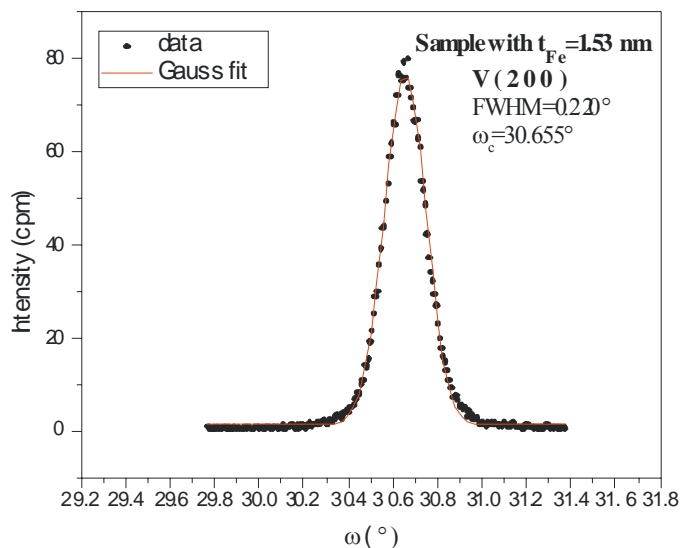

( e )

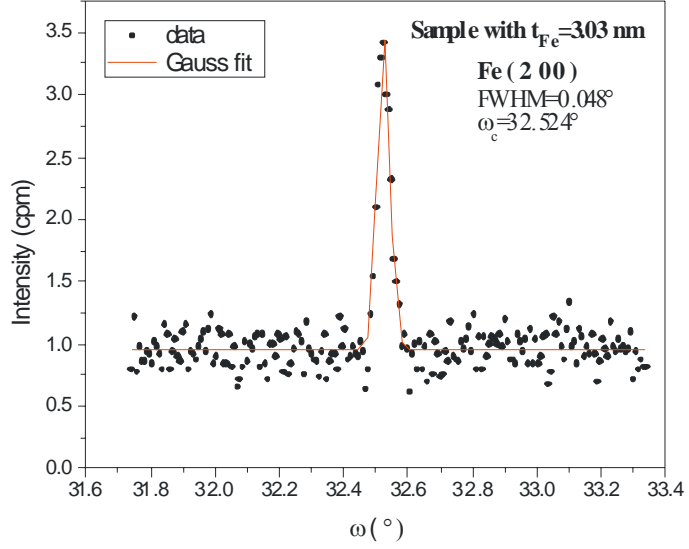

( b )

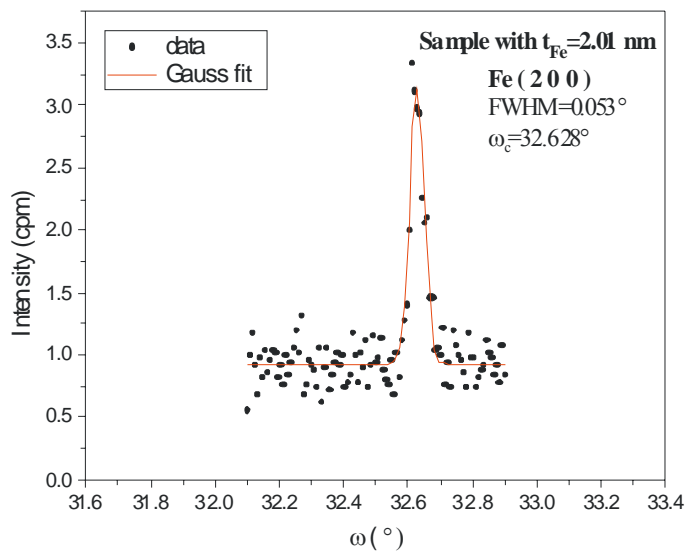

(d)

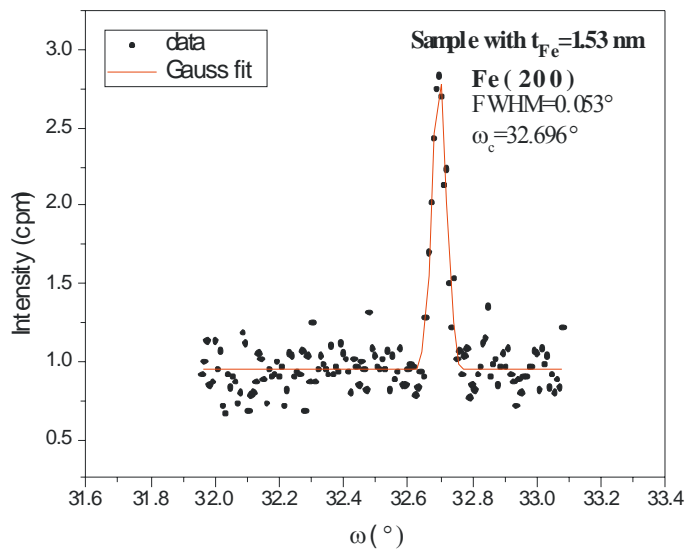

( f)

Figure 4.8 Rocking curves (XRD) of the Vanadium and Iron ( 200 ) Bragg peaks $\left(t_{\mathrm{Fe}}=3.03 \mathrm{~nm}, 2.01 \mathrm{~nm}, 1.53 \mathrm{~nm}\right) . F W H M$ is the full-width at half-maximum of the peak and $\omega_{c}$ is the center position of the peak. 


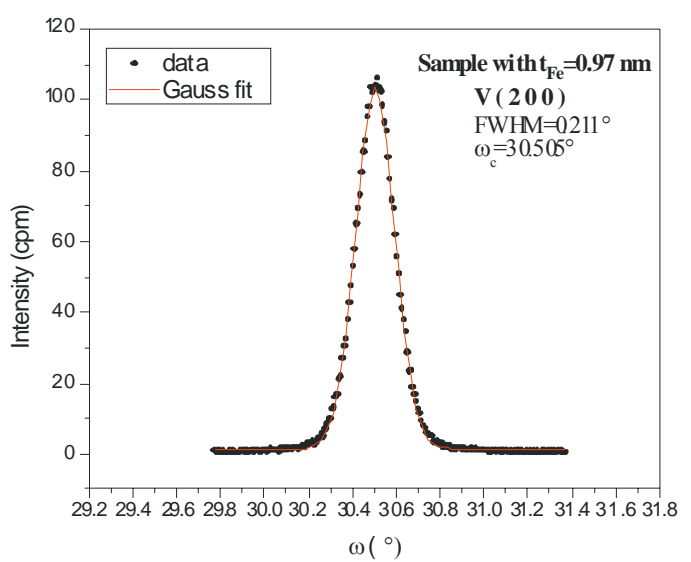

( a )

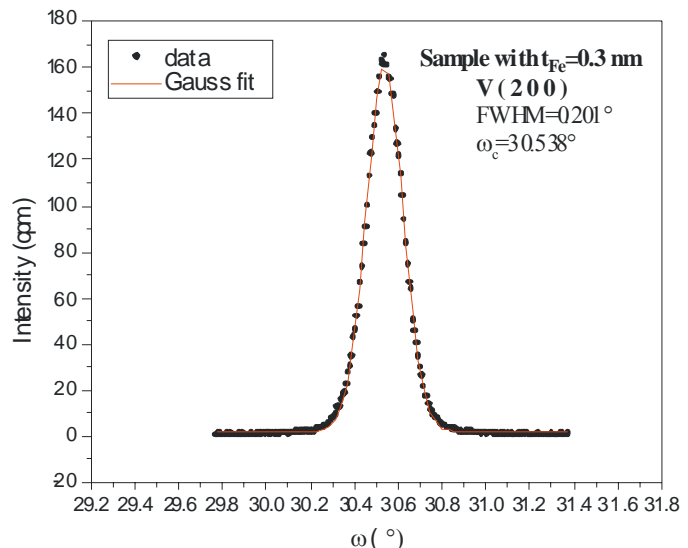

(c)

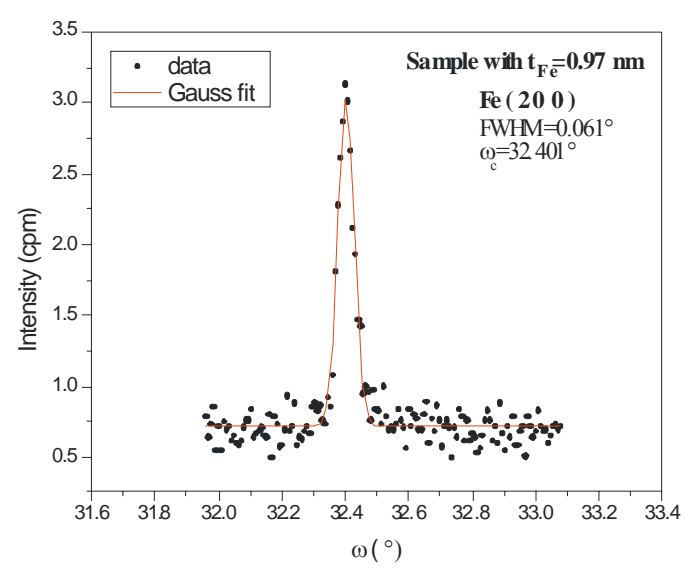

( b )

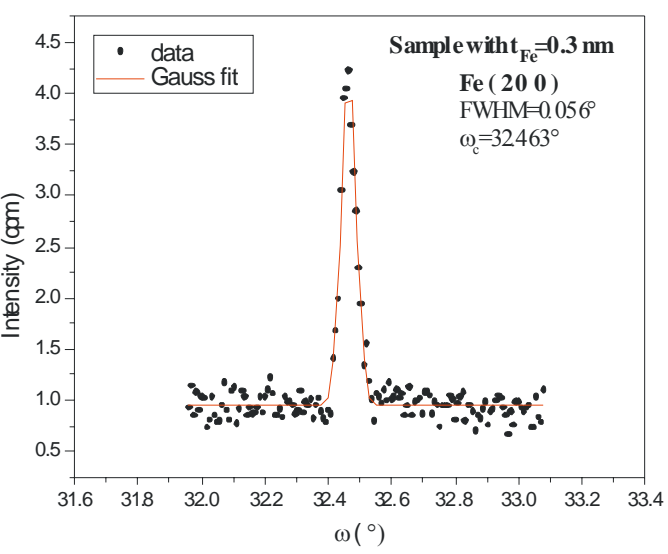

( d )

Figure 4.9 Rocking curves (XRD) of the Vanadium and Iron ( 200 ) Bragg peaks $\left(t_{\mathrm{Fe}}=0.97 \mathrm{~nm}, 0.3 \mathrm{~nm}\right) . F W H M$ is the full-width at halfmaximum of the peak and $\omega_{c}$ is the center position of the peak. 


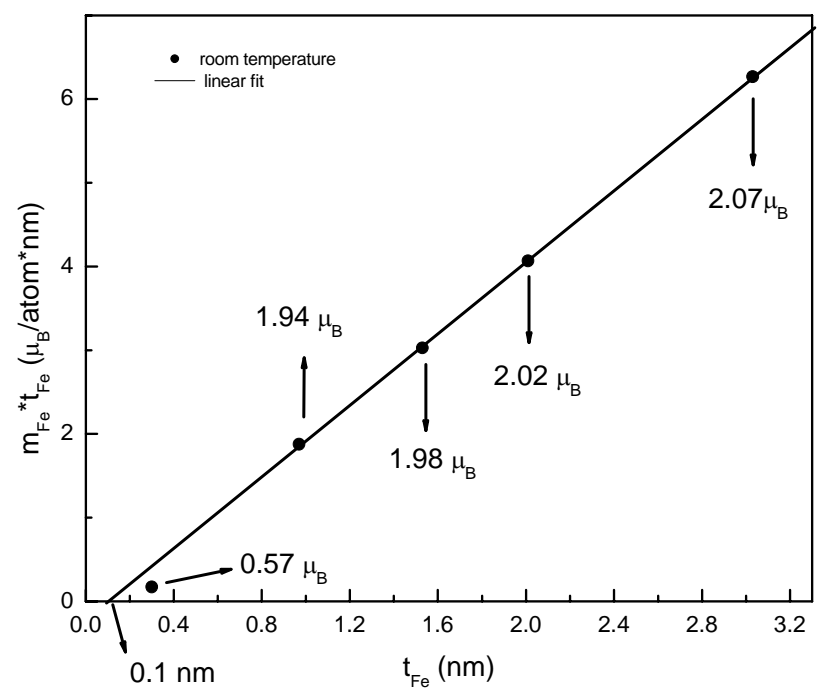

Figure 4.10 The products of the magnetic moment per atom and the Fe film thickness $t_{F e}$ (dots) are plotted versus $t_{F e}$. The solid line is a linear fit to the data points

\subsubsection{Discussion}

PNR has the advantage to provide magnetic and structural information of the sample, whereas XRR is only sensitive to structural properties. However, compared with neutrons $\mathrm{x}$-rays are more sensitive to surface roughness because of the larger accessible $q$-range. The maximum $q$ value $\left(q_{\max }=4.94 \mathrm{~nm}^{-1}\right)$ that could be reached in the XRR measurements is much larger than that $\left(q_{\max }=0.73 \mathrm{~nm}^{-1}\right)$ in the PNR measurements. The influence of the roughness of the Vanadium oxide layer on the neutron and x-ray reflectivities is shown in Figure 4.12. The neutron reflectivity curves show nearly no change when the roughness was changed from $1.6 \mathrm{~nm}$ to $0.4 \mathrm{~nm}$, but the X-ray reflectivity curve shows a big change for the same situation. Withal, compared with neutrons $\mathrm{x}$-rays have a higher sensitivity to distinguish between the Vanadium oxides, $\mathrm{V}_{2} \mathrm{O}_{5}$ and $\mathrm{V}_{2} \mathrm{O}_{3}$. It can be explained by the scattering length densities of $\mathrm{V}_{2} \mathrm{O}_{5}$ and $\mathrm{V}_{2} \mathrm{O}_{3}$ for neutrons and $\mathrm{x}$-rays, which are shown in Table 4-2. For neutrons the scattering length density of $\mathrm{V}_{2} \mathrm{O}_{5}$ is $3.134 \times 10^{-4} \mathrm{~nm}^{-2}$, which is very close to the scattering length density of $\mathrm{V}_{2} \mathrm{O}_{3}$ that is $3.157 \times 10^{-4} \mathrm{~nm}^{-2}$. For $\mathrm{x}$-rays the scattering length density of $\mathrm{V}_{2} \mathrm{O}_{5}$ is $2.701 \times 10^{-3} \mathrm{~nm}^{-2}$, that of 
$\mathrm{V}_{2} \mathrm{O}_{3}$ is $3.758 \times 10^{-3} \mathrm{~nm}^{-2}$. So, the difference of the scattering length density is more pronounced for $\mathrm{x}$-rays and therefore XRR is more sensitive to distinguish which kind of Vanadium oxide was formed. The influence of the different Vanadium oxides on the neutron and the X-ray reflectivity curves is shown in Figure 4.11. From the neutron reflectivities it is hard to say whether the Vanadium oxide $\mathrm{V}_{2} \mathrm{O}_{5}$ or $\mathrm{V}_{2} \mathrm{O}_{3}$ was formed. However in the case of XRR the difference is very obvious. Assuming the existence of a $\mathrm{V}_{2} \mathrm{O}_{3}$ layer gives much better fit results. Thus, with the help of XRR it was confirmed that the Vanadium oxide $\mathrm{V}_{2} \mathrm{O}_{3}$ was formed. This result is different from Ref [Ada81] where it was stated that the Vanadium oxide was $\mathrm{V}_{2} \mathrm{O}_{5}$, but our results agree with those in Ref [Bie97].

\begin{tabular}{|c|c|c|}
\hline & $\mathrm{V}_{2} \mathrm{O}_{3}\left(\mathrm{~nm}^{-2}\right)$ & $\mathrm{V}_{2} \mathrm{O}_{5}\left(\mathrm{~nm}^{-2}\right)$ \\
\hline Neutron & $3.157 \mathrm{E}-4$ & $3.134 \mathrm{E}-4$ \\
\hline x-ray & $3.758 \mathrm{E}-3$ & $2.701 \mathrm{E}-3$ \\
\hline
\end{tabular}

Table 4-2 Scattering length densities of $\mathrm{V}_{2} \mathrm{O}_{3}$ and $\mathrm{V}_{2} \mathrm{O}_{5}$ for neutrons and $x$ rays.

Comparing the simulation results obtained from PNR and XRR in Table 4-1, different parameter values were found for the samples with Fe layer thicknesses $t_{\mathrm{Fe}}=0.97 \mathrm{~nm}$ and $t_{\mathrm{Fe}}=0.3 \mathrm{~nm}$. For the sample with $t_{\mathrm{Fe}}=0.97 \mathrm{~nm}$ the results for the thickness of the Vanadium capping layer as obtained from PNR are $t_{\mathrm{V}}=9.6 \mathrm{~nm}, t_{\mathrm{V} 203}=0.82 \mathrm{~nm}$, but the results obtained from XRR are $t_{\mathrm{V}}=9.22 \mathrm{~nm}, t_{\mathrm{V} 203}=1.76 \mathrm{~nm}$. For the sample with $t_{\mathrm{Fe}}=0.3 \mathrm{~nm}$ the results obtained from PNR are $t_{\mathrm{V}}=9.9 \mathrm{~nm}, t_{\mathrm{V} 203}=0.84 \mathrm{~nm}$, but the results obtained from XRR are $t_{\mathrm{V}}=9.28 \mathrm{~nm}, t_{\mathrm{V} 203}=1.91 \mathrm{~nm}$. Checking the experimental notebook the answer was easily found. This is due to the fact that the two samples were measured by PNR a week after preparation, but the XRR measurements were carried out two months later. All other samples were measured by PNR and XRR two months after they were prepared. It means that the oxidation procedure of the Vanadium capping layer is very slow and does not reach a stable state after one week. The large roughness values of the $\mathrm{V}_{2} \mathrm{O}_{3}$ layers show that the $\mathrm{V}$ capping layers did not oxidize homogeneously. 


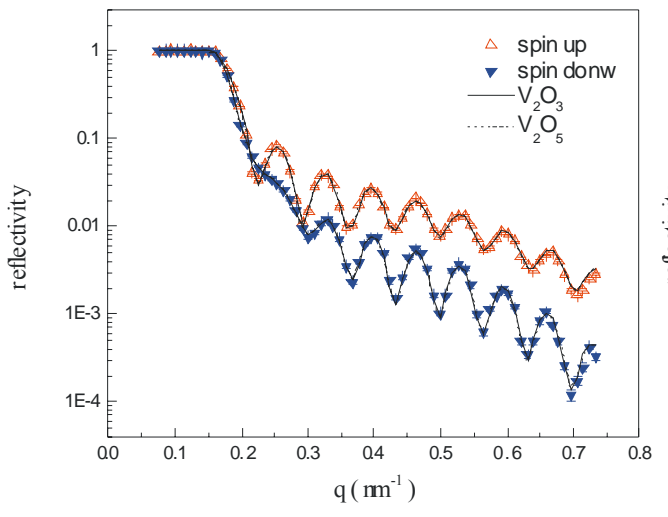

( a )

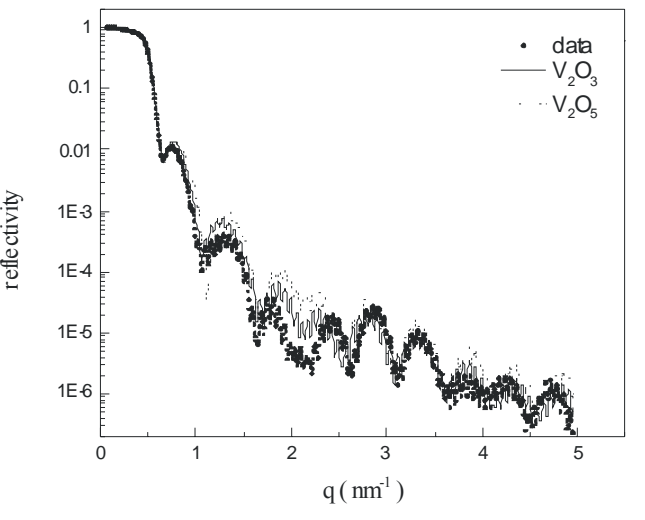

( b )

Figure 4.11 (a) The influence of the different types of Vanadium oxides on the neutron reflectivities; (b) The influence of the different types of Vanadium oxide on the X-ray reflectivities. In both (a) and (b) the solid line is the simulation for $\mathrm{V}_{2} \mathrm{O}_{3}$, the dotted line is the simulation for $\mathrm{V}_{2} \mathrm{O}_{5}$.

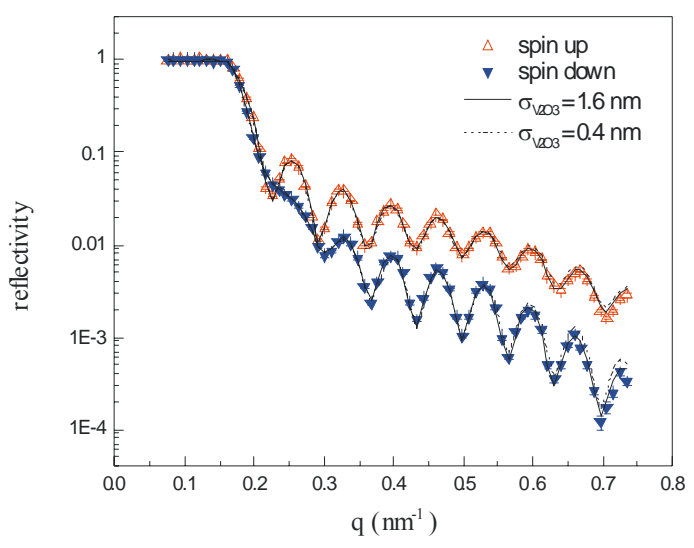

( a )

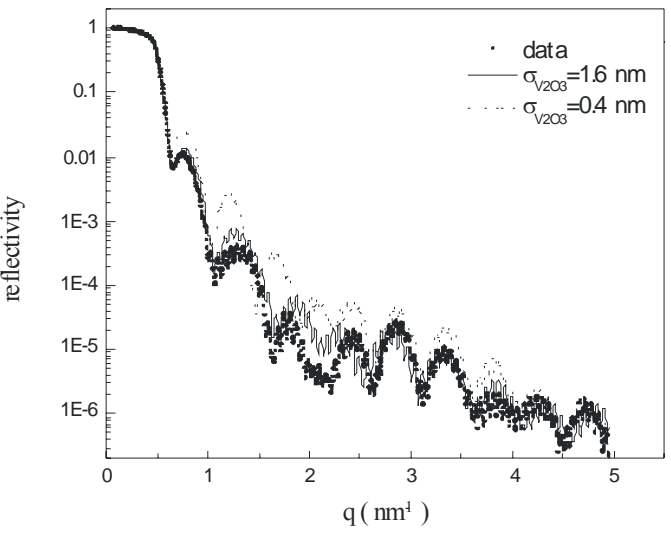

( b )

Figure 4.12 (a) The influence of the roughness of the Vanadium oxides on the neutron reflectivities; (b) The influence of the roughness of the Vanadium oxides on the X-ray reflectivities. In both (a) and (b) the solid line is the simulation where the roughness of the Vanadium oxide is $1.6 \mathrm{~nm}$, the dotted line is the simulation where the roughness of the Vanadium oxide is $0.4 \mathrm{~nm}$. 
The crystalline qualities of all $\mathrm{Fe}$ and $\mathrm{V}$ layers were checked using $\mathrm{x}$-ray diffractometry. In Figure 4.8 and Figure 4.9 the full-width at half-maximum (FWHM) of the $\mathrm{V}(200)$ peak is about $0.2^{\circ}$, the FWHM of the $\mathrm{Fe}(200)$ is about $0.05^{\circ}$. The FWHM value of the $\mathrm{V}(200)$ is larger than that of the $\mathrm{Fe}(200)$. The Fe layer was prepared on top of the $\mathrm{V}$ buffer layer, i.e. in principle the crystalline quality of the thin Fe layer is not expected to be better than that of the $\mathrm{V}$ buffer layer. The broader rocking curves could be due to the different lattice orientations of the $\mathrm{V}$ buffer layer and the $\mathrm{V}$ capping layer, which might be induced by the Fe layer that has a smaller lattice constant than the V layer.

According to Eq. (1-4), the perfect linear fit displayed in Figure 4.10 means that the properties of the thin Fe films are composed of a volume and a surface term. The magnetic moment of the volume is proportional to the slope of the fitted line in Figure 4.10 whereas the deviation from bulk behavior at the interfaces shows up as a nonzero intersection. The magnetic moment value of the inner part of the Fe layer, as determined from the fit, is $2.14( \pm 0.02) \mu_{\mathrm{B}} /$ atom. In order to compare with the theoretical calculation at 0 $\mathrm{K}$ [Wu99], the corresponding result at $0 \mathrm{~K}$ was estimated using Bloch law Eq. (1-1), which was $2.20( \pm 0.02) \mu_{\mathrm{B}} /$ atom agreeing very well with the theoretical result $2.22 \mu_{\mathrm{B}} /$ atom [Wu99]. The $0.1( \pm 0.01) \mathrm{nm}$ intersection with the abscissa corresponds to a total reduction of $-1.5( \pm 0.1) \mu_{\mathrm{B}}$. This reduction is composed of the contributions of the two $\mathrm{Fe} / \mathrm{V}$ interfaces (surface effect) and the temperature-dependent size effect as discussed in Chapter 1. From Eq. (1-3) it is known that $\Delta M_{\text {size }}(T) \propto T^{\frac{3}{2}}$. So, when $T$ is approaching $0 \mathrm{~K}$, the influence arising from the size effect is nearly zero. An investigation has been done by Fritzsche et al. [Fri98] on a sample with $0.85 \mathrm{~nm}$ Fe layer on a V(100) substrate. The result showed that the magnetic moment of the Fe layer did not change when the measuring temperature was changed from $300 \mathrm{~K}$ to $2 \mathrm{~K}$. It means for this kind of samples the size effect at room temperature is so small that its influence could be omitted when $T$ was changed from $300 \mathrm{~K}$ to $2 \mathrm{~K}$. Thus a conclusion can be derived that in the present work the reduction of $-1.5( \pm 0.1) \mu_{\mathrm{B}}$ mentioned above is mainly due to the contribution of the two $\mathrm{Fe} / \mathrm{V}$ interfaces. Assuming that both interfaces are equal, well then, the reduction of the magnetic moment at each $\mathrm{Fe} / \mathrm{V}$ interface is $-0.75( \pm 0.05) \mu_{\mathrm{B}}$ (about 35\% compared with the Fe bulk value), which is in agreement with the theoretical calculations [Ham84, Izq99] and the experimental results [Hos84, 
Tom97, Wa194]. However, the PNR data cannot distinguish between an antiparallel alignment of the $\mathrm{V}$ atoms or a reduced magnetic moment of the interfacial Fe layer.

Furthermore, an additional reduction of the magnetic moment was seen in Figure 4.10 for the case of the Fe layer with $t_{\mathrm{Fe}}=0.3 \mathrm{~nm}$. This data point deviates strongly from the fit line. The explanation may be found in a reduced Curie temperature $T_{\mathrm{c}}$ close to the room temperature, which occurs in very thin films and causes the decrease of the magnetization of the fims. In order to check it, temperature-dependent PNR measurements were performed on this sample.

\subsection{Temperature-dependent PNR measure- ments}

\subsubsection{Motivation}

As mentioned above, the data point $\left(m_{\mathrm{Fe}}{ }^{*} t_{\mathrm{Fe}}\right)$ for the $0.3 \mathrm{~nm}$ Fe layer deviates from the fit line as can be seen in Figure 4.10. This could be due to a reduced Curie temperature which occurs in very thin films. In order to get a deeper insight temperature-dependent PNR measurements were performed.

\subsubsection{Measurements and results}

The ex-situ temperature-dependent PNR measurements were carried out at the reflectometer V6 at the Hahn-Meitner-Institut. The sample with $0.3 \mathrm{~nm}$ Fe layer was cooled by a cryostat, where the temperature could be changed between $300 \mathrm{~K}$ and $10 \mathrm{~K}$.

From the ex-situ PNR and XRR measurements mentioned above the structure parameters of this sample have been derived. Due to the ultrathin $\mathrm{Fe}$ layer thickness the difference between the spin-up neutron reflectivity and spin-down neutron reflectivity is very small as can be seen in Figure 4.5. In order to measure the sample during a limited beam time with reasonable statistics the measurements were carried out in the small- $q$ region $\left(0.5224 \mathrm{~nm}^{-1}\right.$ $<\mathrm{q}<0.5694 \mathrm{~nm}^{-1}$ ), where the splitting for spin-up and spin-down neutrons is large. This region includes one Bragg peak. The sample was measured at 10 $\mathrm{K}, 100 \mathrm{~K}, 200 \mathrm{~K}$ and $300 \mathrm{~K}$. The spin asymmetry defined in Eq.(2-19) is very 
sensitive to the magnetic component. It represents the reflectivity difference caused by the spin-dependent magnetic interaction. In order to check how the magnetic moments of the $\mathrm{Fe}$ atoms change with temperature the spin asymmetries for different temperatures were derived from the data of the reflectivities and were shown in Figure 4.13. The different lines are simulation results for the magnetic component at different temperatures. The magnetic moments derived at different temperatures are given in Table 4-3 and shown in Figure 4.14.

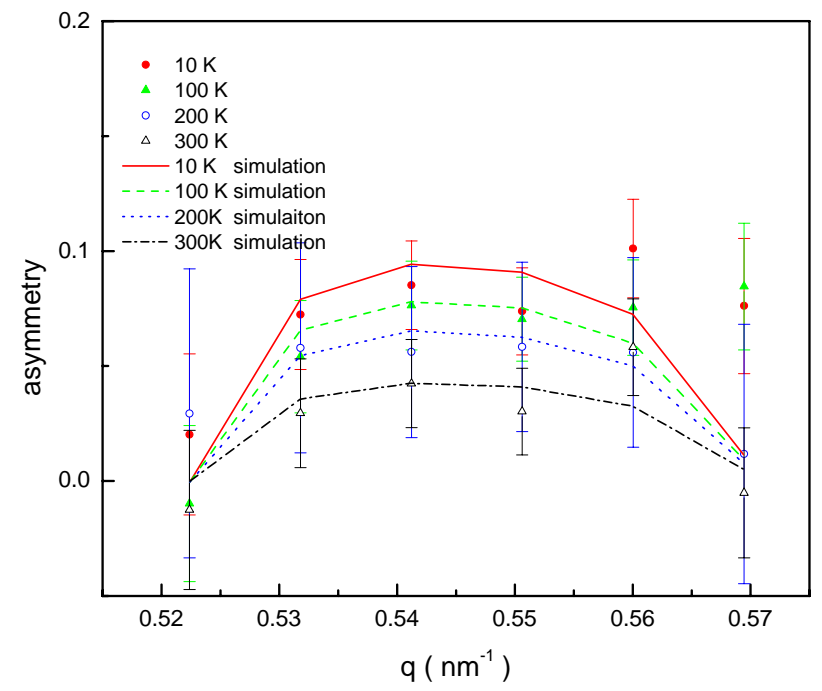

Figure 4.13 Spin asymmetries of the sample with $t_{F e}\left(t_{F e}=0.3 \mathrm{~nm}\right)$ as obtained from the temperature-dependent ex-situ PNR measurements at 10 $\mathrm{K}, 100 \mathrm{~K}, 200 \mathrm{~K}$ and $300 \mathrm{~K}$. The different kinds of lines are simulations corresponding to the measurements at different temperatures.

\begin{tabular}{|c|c|c|c|c|}
\hline$T(\mathrm{~K})$ & 10 & 100 & 200 & 300 \\
\hline$m_{\mathrm{Fe}}\left(\mu_{\mathrm{B}} /\right.$ atom $)$ & 1.28 & 1.06 & 0.88 & 0.56 \\
\hline
\end{tabular}

Table 4-3 Simulation results for the data of the temperature-dependent exsitu PNR measurements. 


\subsubsection{Discussion}

The existence of a net magnetization of the Fe layer with $t_{\mathrm{Fe}}=0.3 \mathrm{~nm}$ indicates the existence of long-range magnetic order in ultrathin films. The data points in Figure 4.14 show an increase of the magnetic moment as the temperature is changed from $300 \mathrm{~K}$ to $10 \mathrm{~K}$. The data points could be fitted very well using a power-law equation of the form $\mathrm{M}(\mathrm{T})=1.28 \times(1-\mathrm{T} / 398)^{0.57}$. This fit does not tell us the true critical exponent because, for the determination of the critical exponent, the sample has to be measured at a temperature very close to $T_{\mathrm{c}}$ [Koh92], the temperatures region between $300 \mathrm{~K}$ to $10 \mathrm{~K}$ is far away from $T_{\mathrm{c}}$. In addition, the $\mathrm{V}$ buffer layer and the $\mathrm{V}$ capping layer have an induced magnetization which is antiparallel to that of the Fe layer, and the values of the magnetic moments measured by PNR are just averages over the total magnetic contribution. But this power-law equation gives us a good estimation for the Curie temperature $\left(T_{\mathrm{c}} \approx 400 \mathrm{~K}\right)$.

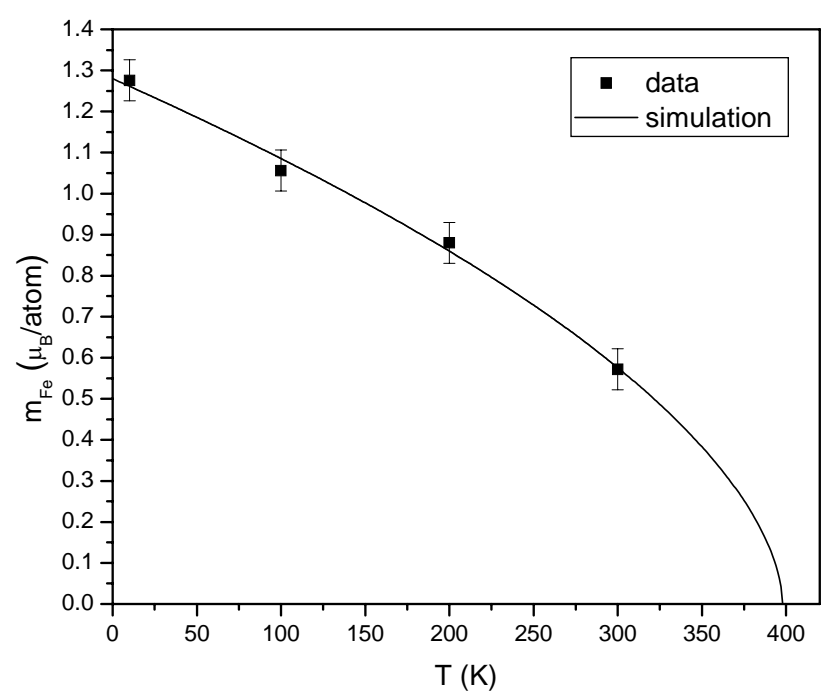

Figure 4.14 Temperature-dependent PNR measurements on the sample with $t_{F e}=0.3 \mathrm{~nm}$. 
In Figure 4.10 an obvious deviation from the fit line was found when the sample of the Fe layer thickness $t_{\mathrm{Fe}}=0.3 \mathrm{~nm}$ was measured at room temperature. However, as shown above the magnetic moment of the Fe layer of the sample is much higher at $10 \mathrm{~K}$ and the product $m_{\mathrm{Fe}}{ }^{*} t_{\mathrm{Fe}}$ at $10 \mathrm{~K}$ is very close to the fitting line. This is shown in Figure 4.15: the triangle represents the data point obtained at $10 \mathrm{~K}$. The data point comes back to the fit line at $10 \mathrm{~K}$ ! That proves again that the reduction of $1.5 \mu_{\mathrm{B}}$ obtained from the intersection of the fit line in Figure 4.10 is mainly due to the surface effect, the size effect at room temperature is so small that it can be omitted in this work. Otherwise, the data point should be above the fit line.

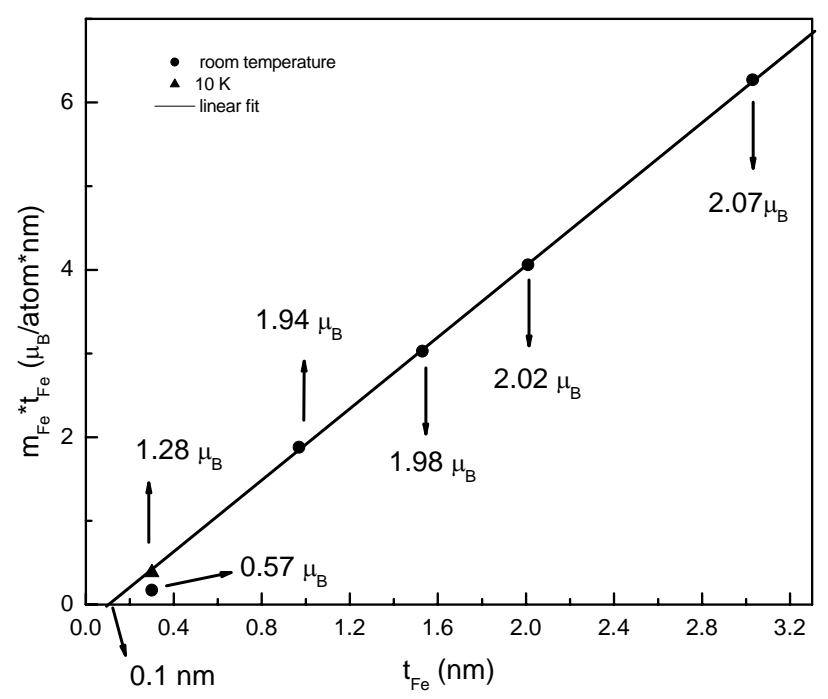

Figure 4.15 The products of the magnetic moment per atom and the Fe film thickness $t_{F e}$ are plotted versus $t_{F e}$. The dots represent the data obtained at room temperature and the triangle is the data obtained at $10 \mathrm{~K}$. The solid line is a linear fit to the data points. 


\subsection{Conclusion}

The $\mathrm{V} / t_{\mathrm{Fe}} \mathrm{Fe} / \mathrm{V}(100)$ system $\left(t_{\mathrm{Fe}}=0.3,0.97,1.53,2.01\right.$ and $\left.3.03 \mathrm{~nm}\right)$ was investigated by ex-situ PNR and XRR. The perfect linear fit of the product $m_{\mathrm{Fe}}{ }^{*} t_{\mathrm{Fe}}$ as a function of the Fe layer thickness $t_{\mathrm{Fe}}$ in the region of $0.97 \leq t_{\mathrm{Fe}}$ $\leq 3.03 \mathrm{~nm}$ in Figure 4.10 proves the general concept for thin films that the magnetic properties are composed of a volume and a surface term. The magnetic moment of the inner part of the Fe layer is $2.14( \pm 0.02) \mu_{\mathrm{B}} /$ atom. The reduction of the magnetic moment at each Fe/V interface is $-0.75( \pm 0.05) \mu_{\mathrm{B}}$. The data point for the product $\left(m_{\mathrm{Fe}}{ }^{*} t_{\mathrm{Fe}}\right)$ of the $0.3 \mathrm{~nm} \mathrm{Fe}$ layer obtained at room temperature deviates from the fit line as can be seen in Figure 4.10. The temperature-dependent measurement on this sample has proven that this deviation is mainly due to the reduced Curie temperature in this ultrathin film. From this experiment the Curie temperature was estimated to be approximately $400 \mathrm{~K}$. From the XRR measurements it was proven that the Vanadium capping layer oxidized inhomogeneously and $\mathrm{V}_{2} \mathrm{O}_{3}$ was formed. 


\section{Chapter 5 The $t_{\mathrm{Fe}} \mathrm{Fe} / \mathrm{V}(100)$ system}

\subsection{Motivation}

The goal of the present work is to determine the magnetic properties of the free $\mathrm{Fe}(100)$ film surface as well as to compare the results with those obtained from earlier in-situ PNR measurements on Fe(110) films [Naw99].

PNR is a very powerful technique to investigate the magnetic and structural properties of thin magnetic films and multilayers. But as reported in Chapter 4, most PNR measurements are performed ex-situ. That means the sample is exposed to air prior to the measurement. In order to avoid contamination thin films must be covered by a capping layer. However a capping layer might affect the magnetic properties of the underlying film and makes it impossible to determine the properties of a free surface of a film. In order to avoid this problem a movable UHV chamber was built in-house, which fits in the limited space at the reflectometer V6 at the HMI. In-situ PNR experiments could be performed, i.e. capping layers were not required any more. It is a great challenge to build an MBE system at a neutron scattering instrument and up to now we are the only ones who performed these kind of in-situ PNR experiments at the HMI.

In this chapter in-situ MOKE and PNR measurements on the samples $t_{\mathrm{Fe}}$ $\mathrm{Fe} / \mathrm{V} / \mathrm{MgO}(100)$ with different Fe thicknesses $t_{\mathrm{Fe}}$ are described and the results are presented. Since PNR is sensitive only to the average magnetization of the films, the contributions of the interface and the free surface can not be separated. But in Chapter 4 the contribution of the Fe/V interface has been derived from the ex-situ PNR measurements. So, comparing the results obtained from in-situ and ex-situ experiments provides a way to determine the 
effect arising from the $\mathrm{V} / \mathrm{Fe}(100)$ interface and the free surface of the $\mathrm{Fe}(100)$ film, separately.

Since Bader et al. [Bad86] discovered that the Kerr effect can be used to record hysteresis loops from ultrathin films, the MOKE technique has been widely applied to investigate the magnetism of ultrathin films. In addition, MOKE is a technique that can probe the magnetic properties of a thin film at a relatively small region. This turns it into a good method in order to measure wedge-shaped samples. Thus quantities depending on the layer thickness can be measured in a single sample and at the same time the effects of the variation of other growth parameters are eliminated. Although it is not able to determine the absolute value of the magnetic moment of films in contrast to PNR, in-situ MOKE measurements are less laborious and less expensive than in-situ PNR measurements. Hence the in-situ MOKE measurements were carried out in order to obtain more magnetic information about this type of samples before the in-situ PNR measurements were carried out.

\subsection{In-situ MOKE measurements}

\subsubsection{Experimental setup}

The in-situ MOKE setup is shown in detail in Figure 5.1 for a longitudinal geometry. The sample is placed in the center of the glass cylinder under UHV conditions. The wavelength of the He-Ne laser is $675 \mathrm{~nm}$. The incident angle of the laser beam is $65^{\circ}$ relative to the normal of the sample surface. A lockin amplifier is used in order to increase the sensitivity. To generate the modulated and polarized light, the laser beam passes through a subsequent arrangement of a polarizer 1 , a photoelastic modulator and a second polarizer 2 . The polarizer 1 is adjusted to a s-configuration and the polarizer 2 is adjusted to a p-configuration. The photoelastic modulator is installed between the two polarizers and its direction of polarization is inclined at $45^{\circ}$ with respect to polarizer 1 and 2. Behind polarizer 2 the modulated and p-polarized light hits the sample and is reflected. The reflected light passes through an analyzer and finally is detected by a photodiode. The analyzer is a polarizer in sconfiguration. The offset angle of the analyzer is about $2^{\circ}$.

An external magnetic field is produced by Helmholtz coils which are outside the glass cylinder and cooled by flowing water. During the measurement 
the value of the magnetic field is controlled by a computer. The sample is mounted in the center of the Helmholtz arrangement, where $B_{\max }=0.12 \mathrm{~T}$ can be reached. As shown in Figure 5.1, the magnetic field is parallel to the plane of the surface of the sample and the incident plane of the light. This direction is also the [100] direction of the prepared samples. To decrease the noise caused by vibration the whole chamber is supported by dampers.

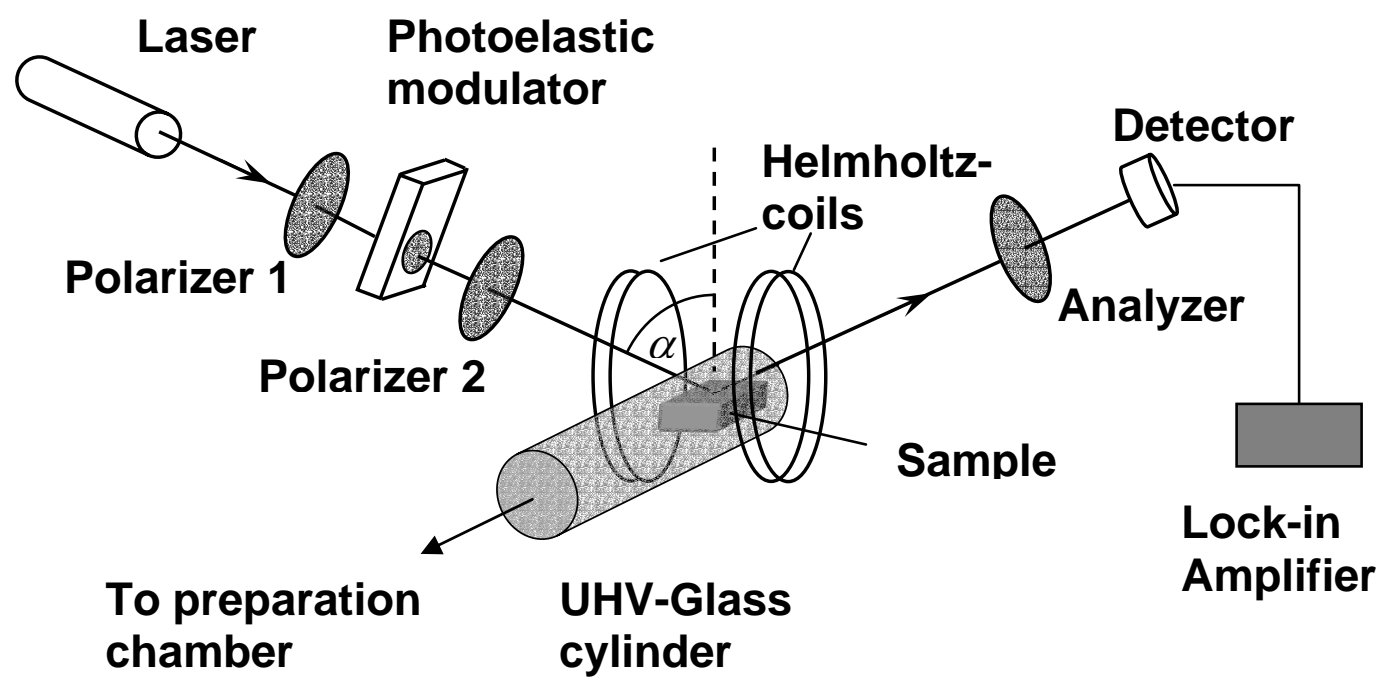

Figure 5.1 In-situ MOKE setup [Sel01]

\subsubsection{Measurements and results}

As mentioned in Chapter 3, for the in-situ MOKE measurements a wedgeshaped sample $t_{\mathrm{Fe}} \mathrm{Fe} / 88 \mathrm{~nm} \mathrm{~V} / \mathrm{MgO}(100)$ was prepared in the stationary MBE (see Figure 3.1). The sketch of the wedge-shaped Fe layer is shown in Figure 3.7, the Fe layer thickness $t_{\mathrm{Fe}}$ changed in steps of $0.1 \mathrm{~nm} / \mathrm{mm}$ from 0.1 $\mathrm{nm}$ to $2 \mathrm{~nm}$, then the thickness was abruptly changed from $2 \mathrm{~nm}$ to $4 \mathrm{~nm}$. This big step serves as a reference position for determining the Fe layer thickness as will be described later. 


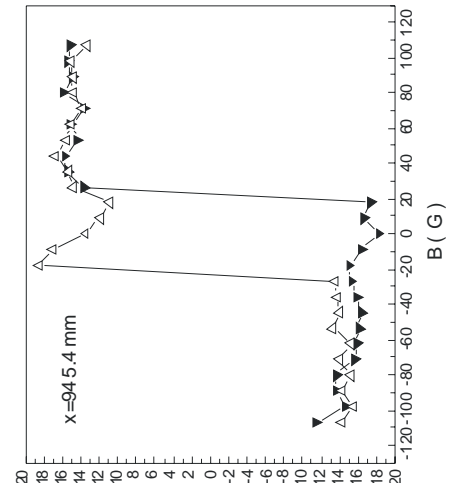

$(\cdot n$ e $)$ ।

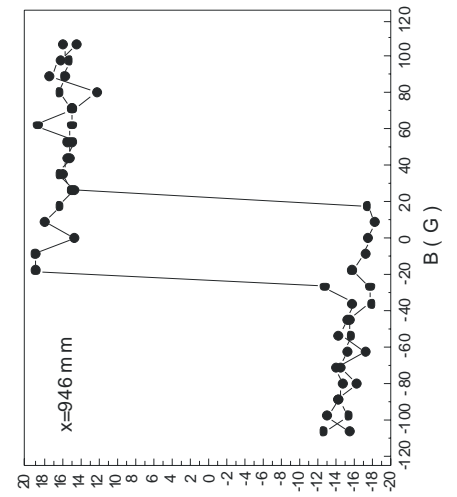

$(\cdot n \bullet)$ ।

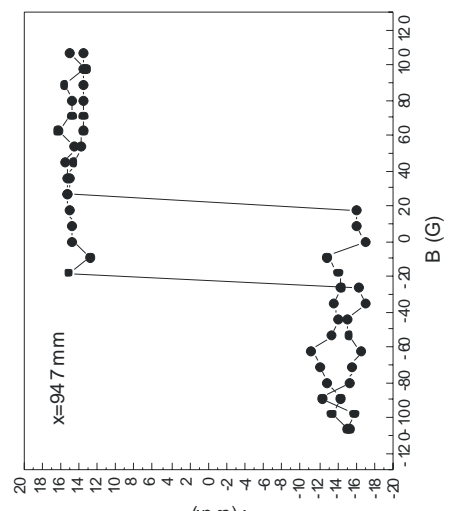

$(n \in)$ ।

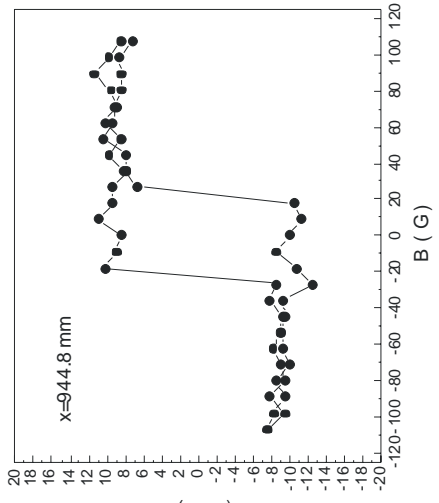

$(n \bullet)$ ।

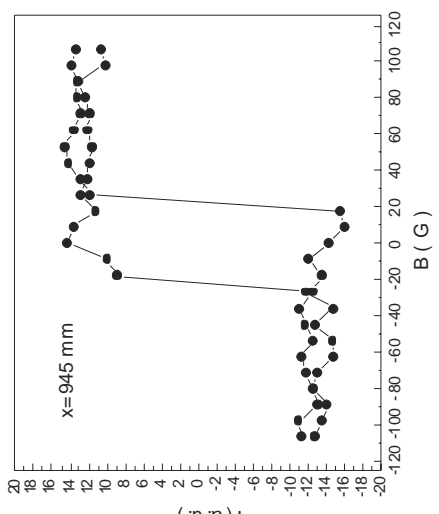

$(n \cdot)$ )।

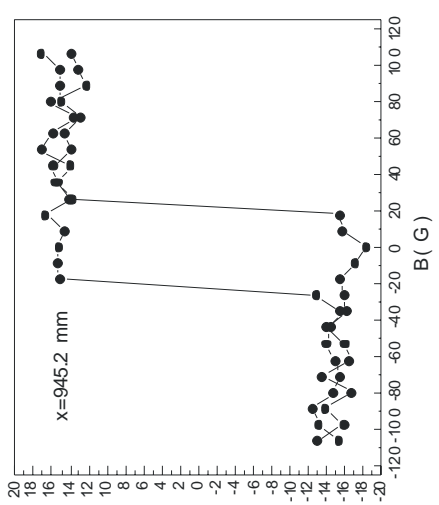

$(\cdot n \cdot)$ ।
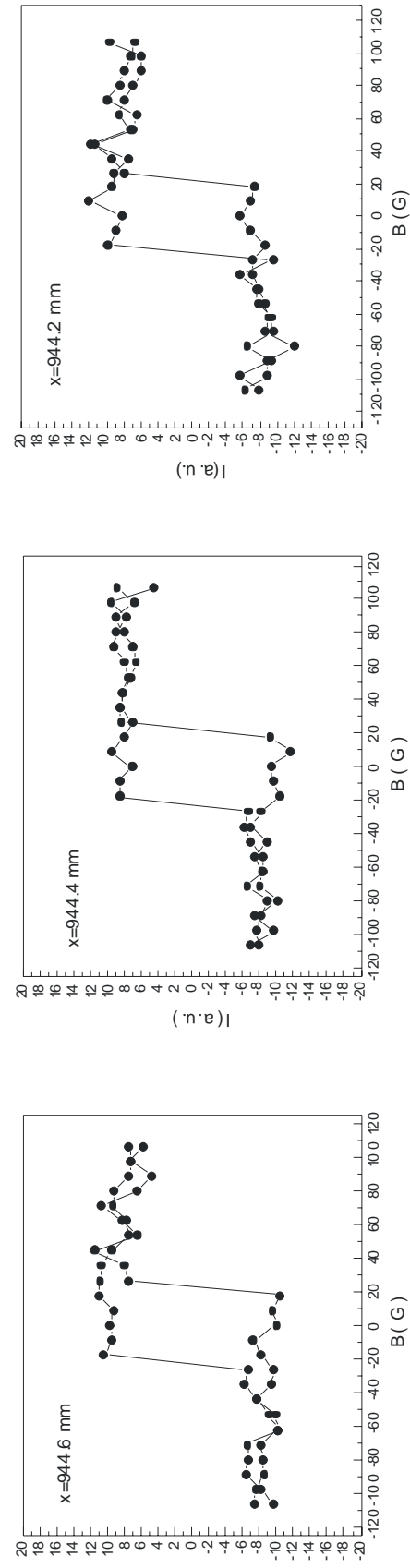

$(\cdot n \cdot e)$ ।

Figure 5.2 MOKE hysteresis loops near the edge between the $2 \mathrm{~nm}$ and $4 \mathrm{~nm}$ Fe layers. $x$ is the value read from the manipulator scale. 


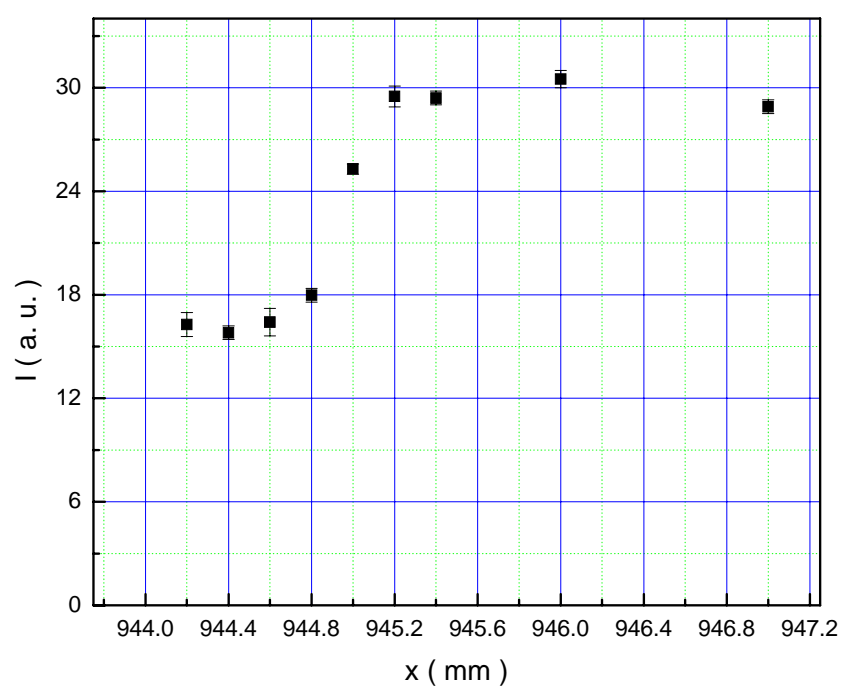

Figure 5.3 Kerr intensities for Fe of different thickness versus the value $x$ read from the manipulator scale near the edge between the $2 \mathrm{~nm}$ and $4 \mathrm{~nm}$ Fe thickness.

After preparation the sample was transferred into the glass cylinder where the MOKE measurement was carried on. The MBE chamber was mounted on a couple of air cushions serving as damping device. Letting air into the cushions inflate the dampers. Thus the whole chamber was slightly lifted and the noise coming from the ground vibration could be reduced. Thereafter the sample angle was adjusted so that the reflected light could be recorded totally by the detector. Afterwards, changing the sample position and measuring the Kerr signal, the edge position between the $2 \mathrm{~nm} / 4 \mathrm{~nm}$ Fe layers was easily found, because the MOKE signal changed considerably due to the big step in the Fe thickness. Then changing the sample position in smaller steps MOKE measurements were performed at the region of the sample near the $2 \mathrm{~nm} / 4$ $\mathrm{nm}$ edge position. MOKE hysteresis loops, i.e. the intensities of the recorded light as a function of the applied field are shown in Figure 5.2 for different positions near the $2 \mathrm{~nm} / 4 \mathrm{~nm}$ edge position. From these measurements the maximum Kerr signals recorded at saturation fields are plotted as a function 
of the measured positions of the sample near the $2 \mathrm{~nm} / 4 \mathrm{~nm}$ edge in Figure 5.3. $\mathrm{x}$ was read from the scale of the manipulator, which gave the information how much the sample position was changed. The motor that changed the sample position was controlled by a computer. The precision was better than $0.01 \mathrm{~mm}$, so its influence on the precision of the sample position could be neglected. From the graph in Figure 5.3 the resolution of the MOKE measurements was inferred, which was smaller than $0.6 \mathrm{~mm}$ including the effect of the size of the laser spot and the edge sharpness of the neighboring steps. One should mention that the edge sharpness is determined by the distance between the shutter and the sample. For different sample preparations it may be different due to a slight change of this distance, but for the same sample it is fixed during the whole procedure, so the resolution should be the same at different positions for the same sample.

In Figure 5.3 it can be seen that the Kerr intensities are stable when the sample position $\mathrm{x} \leq 944.6 \mathrm{~mm}$ or $\mathrm{x} \geq 945.2 \mathrm{~mm}$. Therefore it could be estimated that the center of the $2 \mathrm{~nm} / 4 \mathrm{~nm}$ edge was at $\mathrm{x}=944.9 \mathrm{~mm}$. Accordingly at $\mathrm{x}=944.4 \mathrm{~mm}$ the Fe thickness should be $2 \mathrm{~nm}$, at $\mathrm{x}=943.4 \mathrm{~mm}$ the $\mathrm{Fe}$ thickness should be $1.9 \mathrm{~nm}$ and so on. Thus, the subsequent MOKE measurements were performed at different positions corresponding to different $\mathrm{Fe}$ thickness. The MOKE hysteresis loops obtained from different Fe thicknesses are shown in Figure 5.4 and Figure 5.5. From these data the relation between the MOKE signal intensities and the Fe thickness was obtained. The maximum Kerr intensities are plotted versus the Fe thickness in Figure 5.6. A linear fit at a region of $0.9 \mathrm{~nm} \leq t_{\mathrm{Fe}} \leq 2 \mathrm{~nm}$ results in a slope of $8.1( \pm 0.4) \mathrm{nm}^{-1}$ and an intersection with the abscissa at $0.034( \pm 0.07) \mathrm{nm}$.

From the MOKE hysteresis loops obtained from different Fe thicknesses, shown in Figure 5.4 and Figure 5.5, a plot of the thickness dependence of the coercivity $H_{\mathrm{c}}$ was extracted and shown in Figure 5.7. 

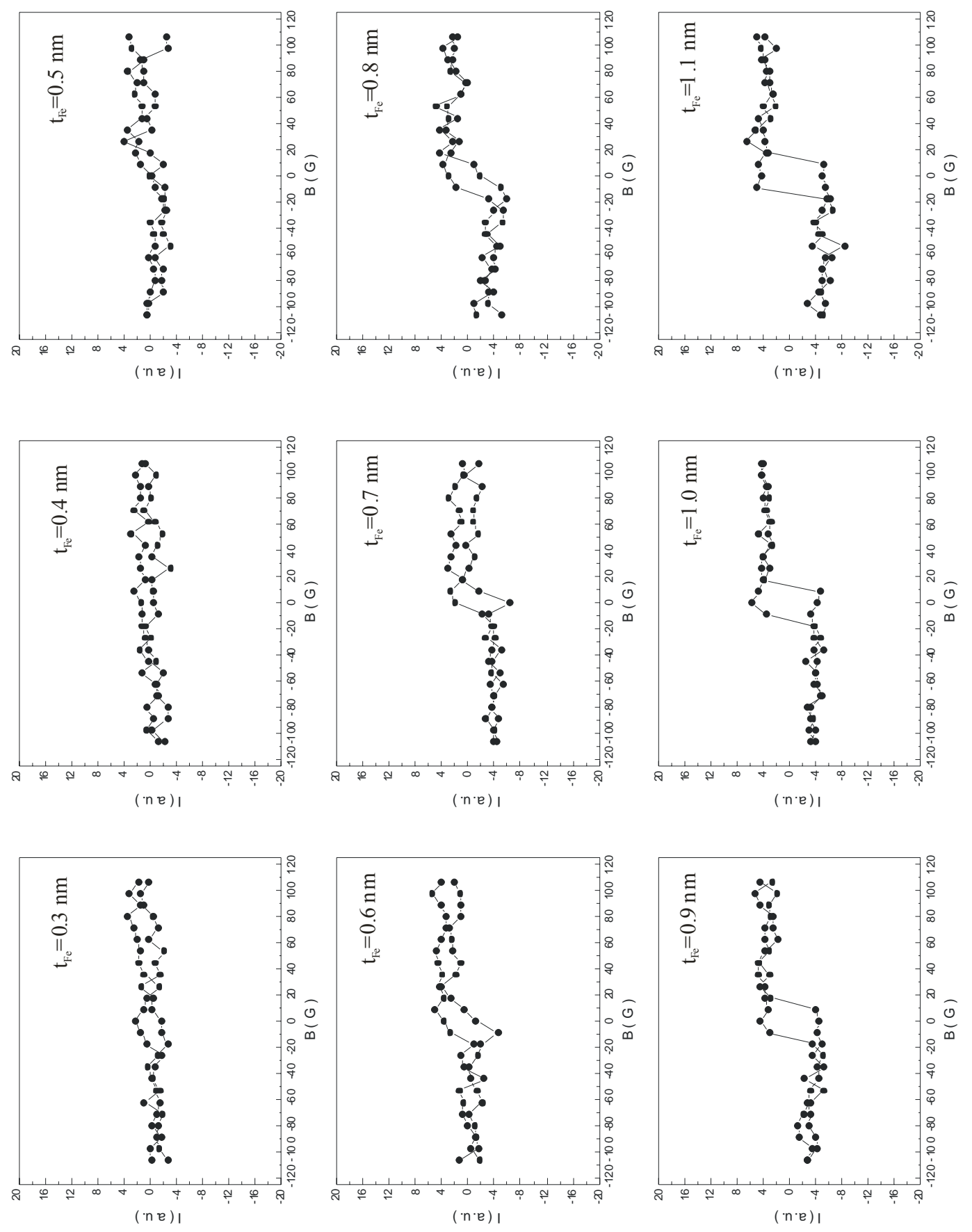

Figure 5.4 MOKE hysteresis loops of Fe layers with different thicknesses. 

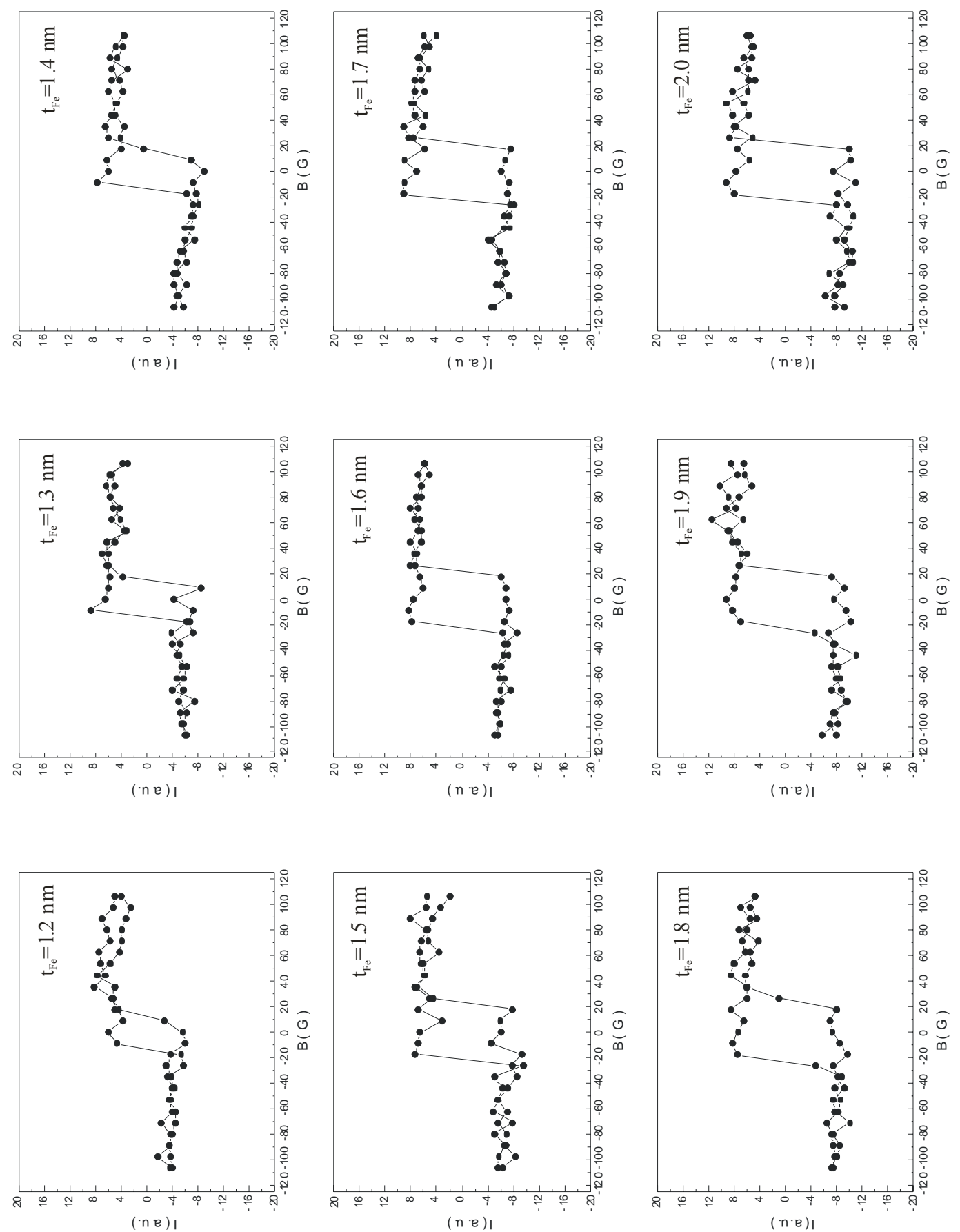

Figure 5.5 MOKE hysteresis loops of Fe layers with different thicknesses. 


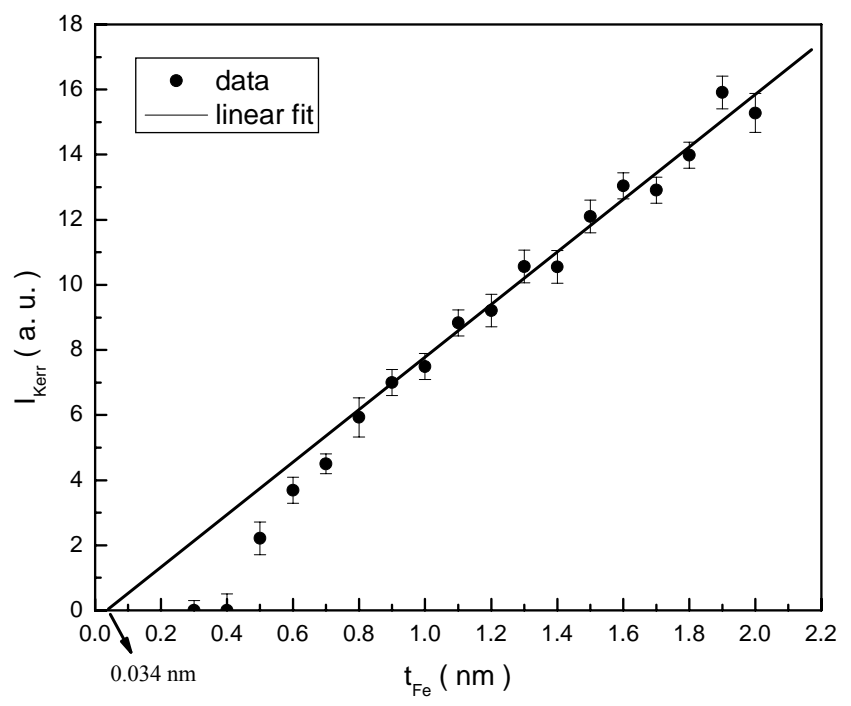

Figure 5.6 Kerr intensities of different Fe layers versus the Fe thickness $t_{F e}$. The solid line is a linear fit to the data points at a region $0.9 \mathrm{~nm} \leq$ $\mathrm{t}_{\mathrm{Fe}} \leq 2 \mathrm{~nm}$.

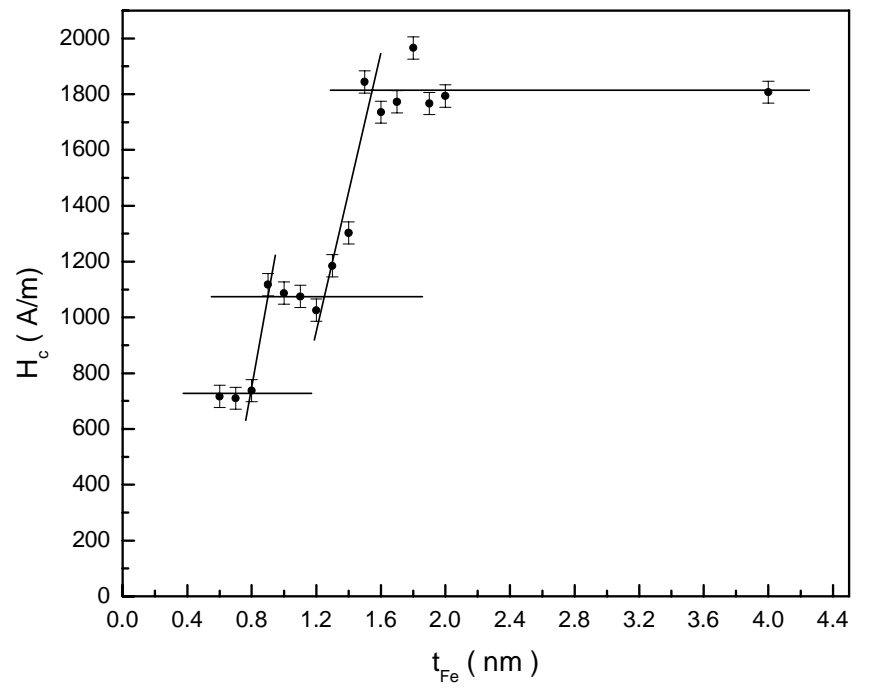

Figure 5.7 Thickness dependence of the coercivity for Fe/V(100) grown at room temperature. Dots are the data points, the solid lines are the fit lines. 


\subsubsection{Disscussion.}

The square shaped heysteresis loops in Figure 5.4 and Figure 5.5 show that the easy axis is aligned in-plane along the [100] direction for $t_{\mathrm{Fe}} \geq 0.6 \mathrm{~nm}$. From Eq. (2-25) it is known that $I_{\text {Kerr }}$ is proportional to the magnetization of the sample. So, from the dependence of $I_{\text {Kerr }}$ on the Fe thickness $t_{\mathrm{Fe}}$ it can be concluded how the magnetization changes with the Fe film thickness $t_{\mathrm{Fe}}$. In Figure 5.6 the graph can be separated into two parts. The first part includes $0.8 \mathrm{~nm} \leq t_{\mathrm{Fe}} \leq 2 \mathrm{~nm}$ where the linear increase in $I_{\text {Kerr }}$ with the thickness $t_{\mathrm{Fe}}$ proves the general concept for thin films that the properties are composed of a volume and a surface term. The magnetic moment of the volume is proportional to the slope of the fitted line of $8.1 \mathrm{~nm}^{-1}$. Whereas the deviation from bulk behavior at the surfaces shows up as a nonzero intersection of $0.034( \pm 0.07) \mathrm{nm}$, which is corresponding to a total reduction of the magnetic moment of $-0.51( \pm 1) \mu_{\mathrm{B}}$. The second part includes $t_{\mathrm{Fe}} \leq 0.8 \mathrm{~nm}$ where the data was deviate from linear behavior.

For the first part the intersection denotes the contribution arising from both the $\mathrm{Fe} / \mathrm{V}(100)$ interface and the Fe surface. An increase in the magnetization at the Fe surface was predicted by theories [Fre85, Fu85, Sto95, Veg95] and was proven by experiments [Elm89, Tam90]. A reduction in the magnetization at the $\mathrm{Fe} / \mathrm{V}$ interface was predicted by theories [Coe95, Spi00, Izq99] and was proven by experiments [Dud96, Gra98, Pou97, Tom97]. That means the magnetization of the free Fe surface is different from that of the $\mathrm{Fe} / \mathrm{V}(100)$ interface. But it is difficult to distinguish between the contribution of the $\mathrm{Fe} / \mathrm{V}(100)$ interface and the contribution of the free Fe surface here since PNR is only sensitive to the average magnetization of the films. However the similar samples with V capping layers were measured by ex-situ PNR as reported in Chapter 4 and the conclusion was that the reduction of the magnetic moment caused by the $\mathrm{Fe} / \mathrm{V}(100)$ interface is $-0.75( \pm 0.05) \mu_{\mathrm{B}}$. Thus, an enhancement of $+0.24( \pm 1) \mu_{\mathrm{B}}$ of the magnetic moment at the free Fe surface was derived.

For the second part, i.e. for Fe thicknesses smaller than $0.8 \mathrm{~nm}$ the data points deviate from the linear fit of the first part. These data points occur below the fit line. The same deviation was observed in the ex-situ PNR measurements, as reported in Chapter 4. The temperature-dependent PNR measurements proved that this deviation originated in the reduced Curie temperature $T_{\mathrm{c}}$ of the sample with an Fe layer thickness of $t_{\mathrm{Fe}}=0.3 \mathrm{~nm}$. It has also 
been reported in several papers [Due89, Moo85, Rau89] that the Curie temperature decreased as the film thickness was reduced.

Moreover similar experiments have been performed by Nawrath et al. [Naw99] on a Fe/V(110) sample. The intersection found was $0.4 \mathrm{~nm}$. Compared with the intersection of $0.034 \mathrm{~nm}$ found here the difference of the two values is quite large. It has mentioned that the Fe layers were prepared on substrates with different orientations. But this can not be used to explain the different values. From the theoretical calculations [Izq99] it is expected that the reduced magnetization at the interface of $\mathrm{Fe} / \mathrm{V}(110)$ is smaller than that the $\mathrm{Fe} / \mathrm{V}(100)$ interface. A detailed explanation will be given in the following paragraph in combination with the in-situ PNR results.

From the MOKE hysteresis loops obtained from Fe layers of different thickness, as shown in Figure 5.4 and Figure 5.5 a plot of the thickness dependence of the coercivity $H_{\mathrm{c}}$ was extracted and shown in Figure 5.7. It is very interesting that a four-stage behavior was obtained. The first stage occurs at $0.6 \mathrm{~nm} \leq t_{\mathrm{Fe}} \leq 0.8 \mathrm{~nm}$, where the coercivities are constant $\left(H_{\mathrm{c}}=724\right.$ $\mathrm{A} / \mathrm{m})$. When the Fe thickness changes from $0.8 \mathrm{~nm}$ to $0.9 \mathrm{~nm}$, the coercivities increase abruptly upto the second stage and remain constant $\left(H_{\mathrm{c}}=1074 \mathrm{~A} / \mathrm{m}\right)$ for $0.9 \mathrm{~nm} \leq t_{\mathrm{Fe}} \leq 1.2 \mathrm{~nm}$. It follows the third stage where the coercivities increase linearly from $1074 \mathrm{~A} / \mathrm{m}$ to $1814 \mathrm{~A} / \mathrm{m}$ when the Fe thickness increases from $1.2 \mathrm{~nm}$ to $1.5 \mathrm{~nm}$. Finally, at the fourth stage where the Fe thicknesses are $1.5 \mathrm{~nm} \leq t_{\mathrm{Fe}} \leq 4.0 \mathrm{~nm}$ the coercivities become stable again at a field of $1814 \mathrm{~A} / \mathrm{m}$. Until now many similar researches [Ara88, Bad86, Liu91, Sch93, Xu98] have been done, but this kind of behavior was never found. 


\subsection{In-situ PNR measurements}

\subsubsection{Experimental setup}

In situ PNR measurements were performed at the reflectometer V6 at the Hahn-Meitner-Institut Berlin. At first the sample table and the black shielding box that belong to the standard setup of the V6 were put aside. Then the devices (a movable UHV chamber, a shielding box and a two-dimensional detector, etc.) for the in-situ measurements were arranged. A sketch of the insitu PNR experimental setup is shown in Figure 5.8. Two photos showing the experimental setup of the in-situ experiment are given in Figure 5.9 and Figure 5.10.

A wavelength of $0.466 \mathrm{~nm}$ of the incoming neutron beam was selected using a graphite monochromator. Second-order contaminations were reduced by a liquid-nitrogen-cooled $\mathrm{Be}$ filter. The neutron beam was collimated by two sets of computer controlled cadmium slit systems. The neutrons were polarized by a Si-FeCo supermirror [Kri95] which reflected the spin-up neutrons while the spin-down neutrons could pass through. When spin-up neutrons were needed, a Mezei-type spin flipper was used to reverse the spin direction via Larmor precession. In order to avoid depolarization of the neutrons a guide field was also applied throughout the beam path between the polarizer and the sample.

The sample was prepared using the movable MBE chamber, which has been introduced in Chapter 3. Passing through a glass cylinder flanged to the UHV chamber the neutron beam hit the sample which was kept under ultrahigh vacuum conditions. The angle between the incoming beam and the sample surface was adjusted using a precision rotary feedthrough. A pair of Helmholtz coils mounted outside the glass cylinder provided a horitzontal magnetic field which was directed perpendicular to the reflection plane $\left(\mathrm{B}_{\max }=0.12 \mathrm{~T}\right)$. The coils were cooled by flowing water during operation.

The detector used was a two-dimensional position sensitive detector with a resolution of $1.5 \mathrm{~mm}$, which was placed $1.49 \mathrm{~m}$ away from the sample. The angle of $2 \theta$ was determined precisely by the distance between the reflected beam and the direct beam. 


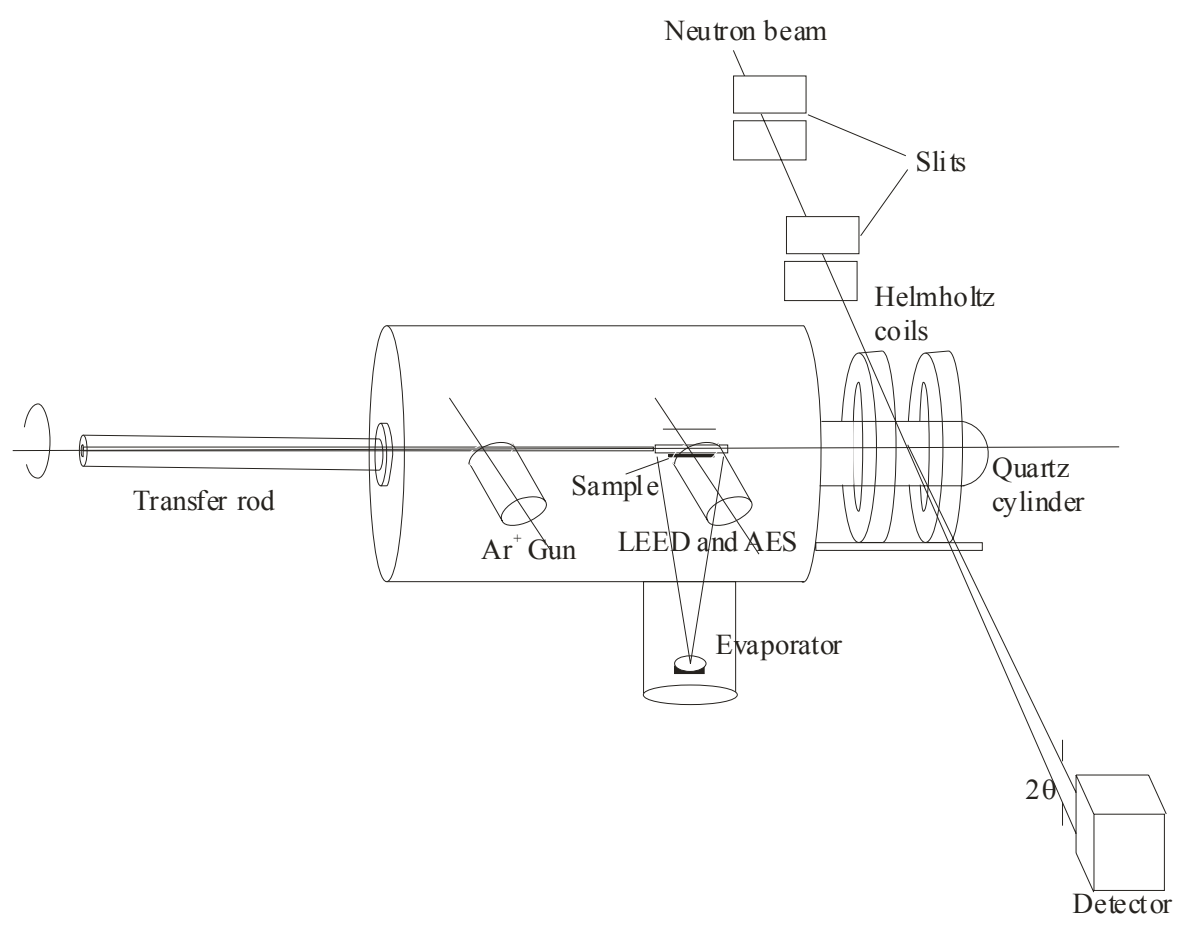

Figure 5.8 Sketch of the in-situ PNR experimental setup. 


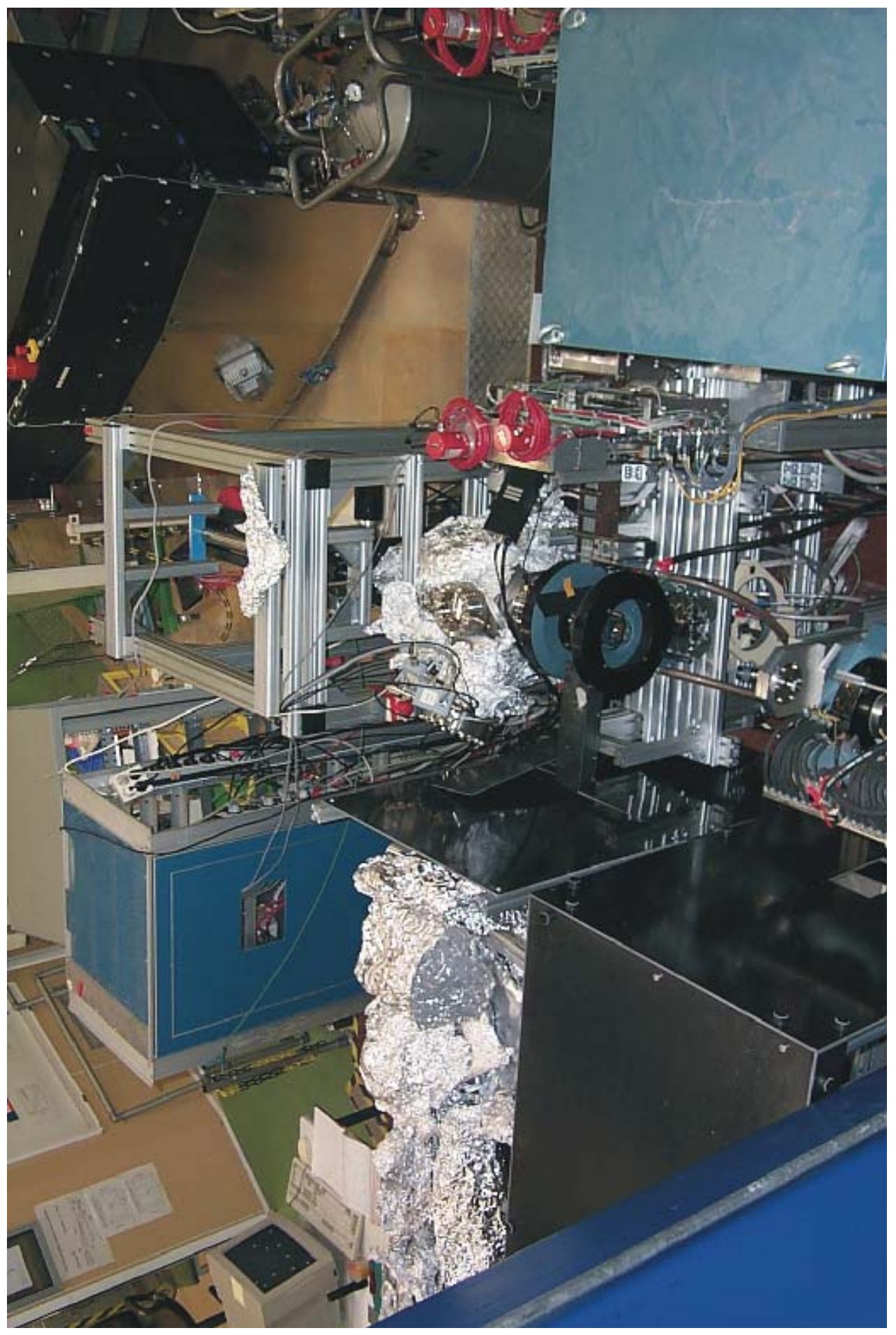

Figure 5.9 In-situ experimental setup 


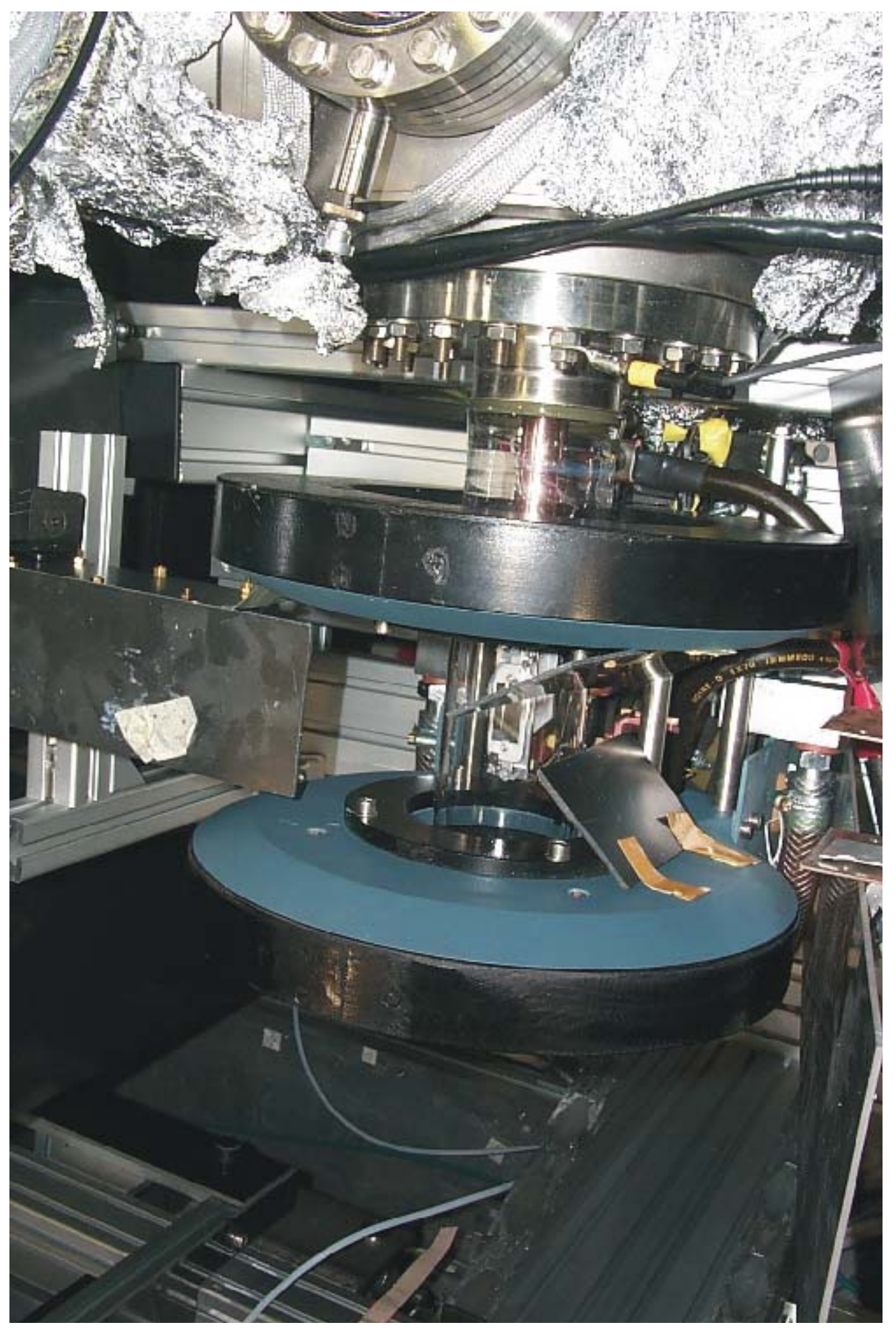

Figure 5.10 Sample, glass cylinder and Helmholtz coils 


\subsubsection{Measurements and results}

The sample was prepared as described in Chapter 3. After preparation the sample was transferred into the glass cylinder of the chamber where the incoming neutron beam passed through. The angle of the sample was adjusted by a motor that was controlled by the neutron instrument software CARESS which was developed at the HMI. The sample height was adjusted roughly by adjusting the height of the chamber to the height of a laser beam previously aligned with the height of the neutron beam path. The fine adjustment of the height and the angle of the sample were done by performing slit height scans and sample rocking scans, respectively.

After each PNR measurement the Fe layer was checked by AES and it was proven that the surface was not contaminated during the measurement. Afterwards, the sample was sputtered for 90 seconds with a Argon beam at a voltage of $370 \mathrm{~V}$. The sputter gun was tilted at an angle of 50 degree with respect to the sample surface. It was checked by AES that the Fe layer had been sputtered away totally. Thereafter the sample was annealed at $700 \mathrm{~K}$ for 20 minutes. Subsequently an $8 \mathrm{~nm} \mathrm{~V}$ layer was deposited on top of it and the sample was annealed again at $700 \mathrm{~K}$ for 20 minutes. Then another $3 \mathrm{~nm}$ of V were prepared on top of it. The evaporation rate was $0.01 \mathrm{~nm} / \mathrm{s}$. Finally an $\mathrm{Fe}$ layer with the desired thickness was prepared on top of the $\mathrm{V}$ buffer layer and measured in-situ using PNR.

The reflectivity and the spin asymmetry curves of the samples obtained from in-situ PNR measurements are plotted in Figure 5.11 and Figure 5.12 as a function of the scattering vector $\vec{q}$, defined as $q=4 \pi \sin \theta / \lambda$, where $\theta$ is the angle of incidence and $\lambda$ is the neutron wavelength. The spin-up reflectivity curves are plotted as up-triangles and the spin-down reflectivity curves are plotted as down-triangles. The spin asymmetries are plotted as dots. PNR is very sensitive to the magnetic component and shows directly the reflectivity difference caused by the spin-dependent magnetic interaction. The analysis was done by a software based on the Parratt formalism. The initial values of the layer thickness were given by the values obtained from the calibrated quartz crystal thickness monitor. Then the parameters were optimized in order to find the best values to fit both the spin-up and spin-down curves. Afterwards, the parameter of the magnetic scattering length density was adjusted slightly to find the optimum value that fitted the spin asymmetry curves. Finally, the layer thickness, roughness and the absolute value of the 
magnetization of the films were fitted. The ultimate simulations are shown in Figure 5.11 and Figure 5.12 as solid lines. The individual parameters of the fitting results are also given in Table 5-1.

From the results of the in-situ PNR measurements, the products of the magnetic moment per atom and the Fe thickness were derived and plotted in Figure 5.13 as a function of the Fe film thickness. The products are proportional to the effective magnetic moments of the Fe films. A linear fit results in a slope of $2.14( \pm 0.04) \mu_{\mathrm{B}} /$ atom and an intersection with the abscissa at $0.03( \pm 0.06) \mathrm{nm}$. 


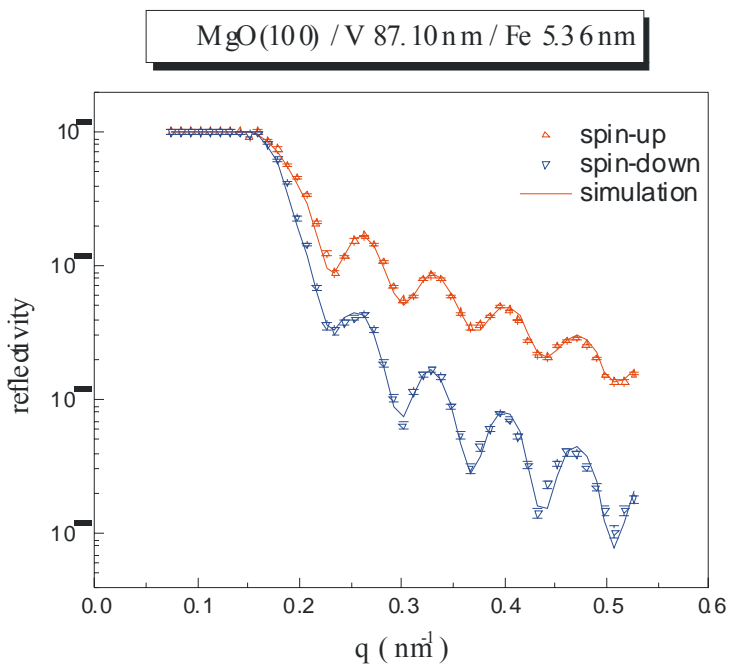

( a )

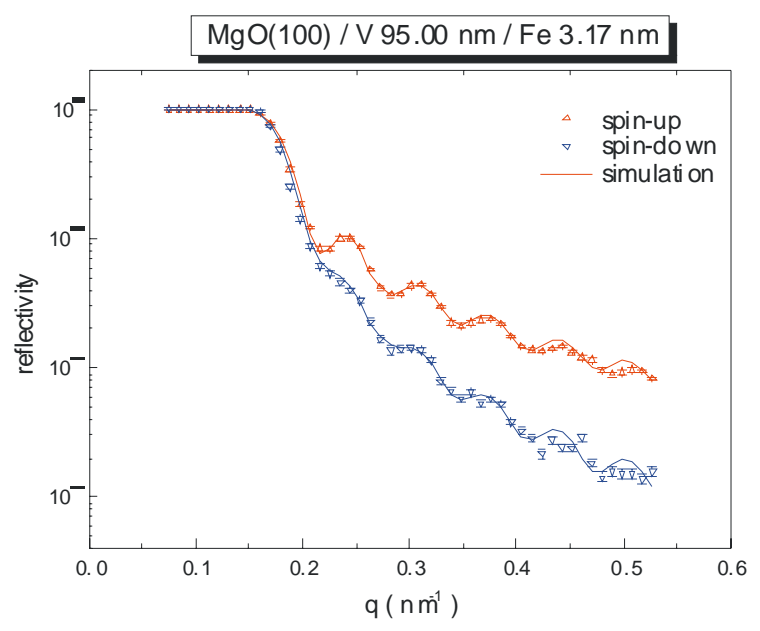

( c )

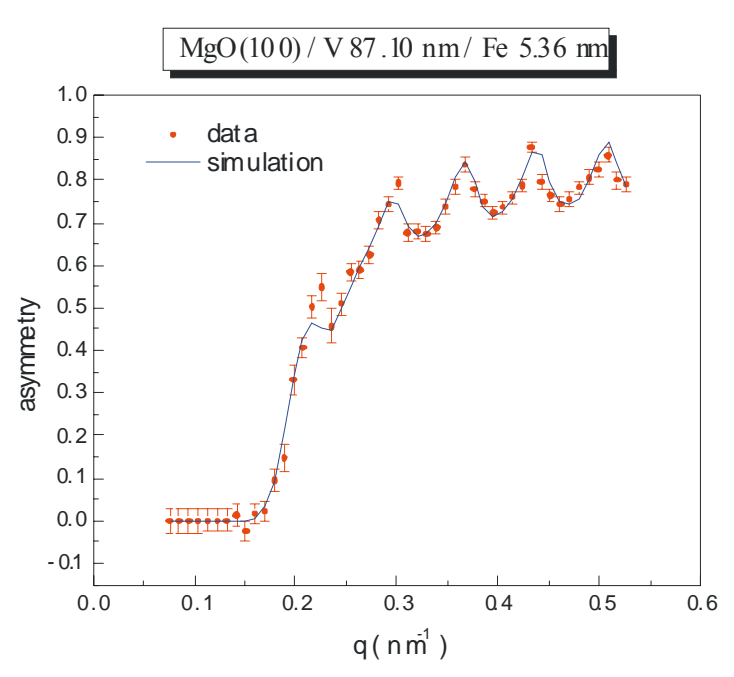

( b )

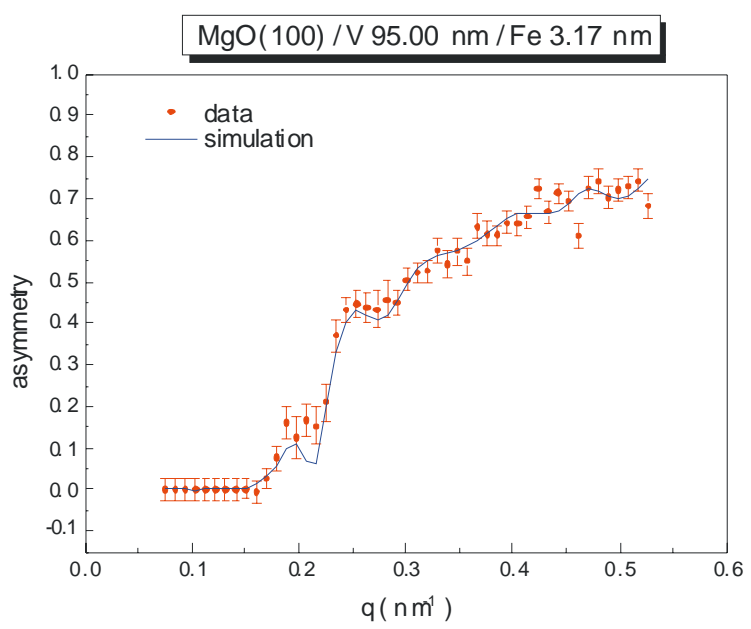

( d )

Figure 5.11 Reflectivity and spin asymmetry curves of the samples with $t_{F e}=$ $5.36 \mathrm{~nm}$ and $t_{F e}=3.17 \mathrm{~nm}$ as obtained from in-situ PNR measurements. The reflectivity curves of the spin-up (spin-down) neutrons are given as up-triangles (down-triangles). The spin asymmetry curves are shown as dots. The solid lines are results from simulations. 


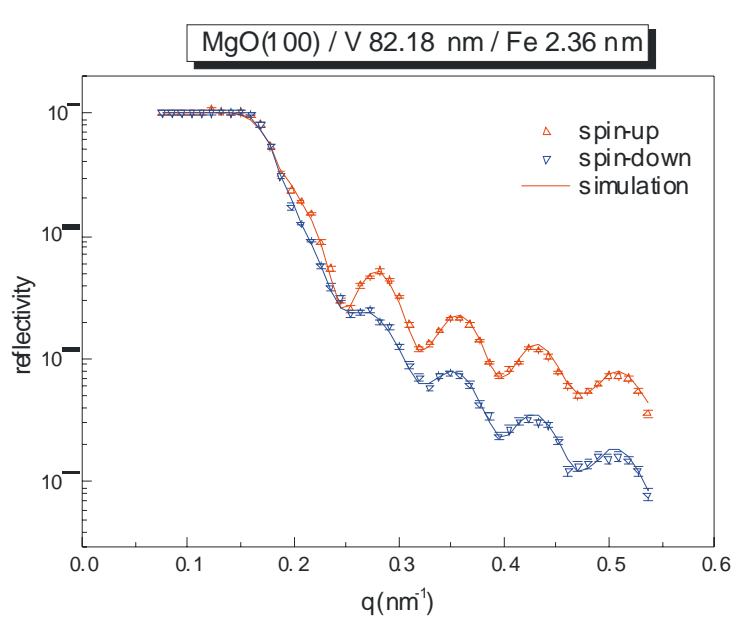

( a )

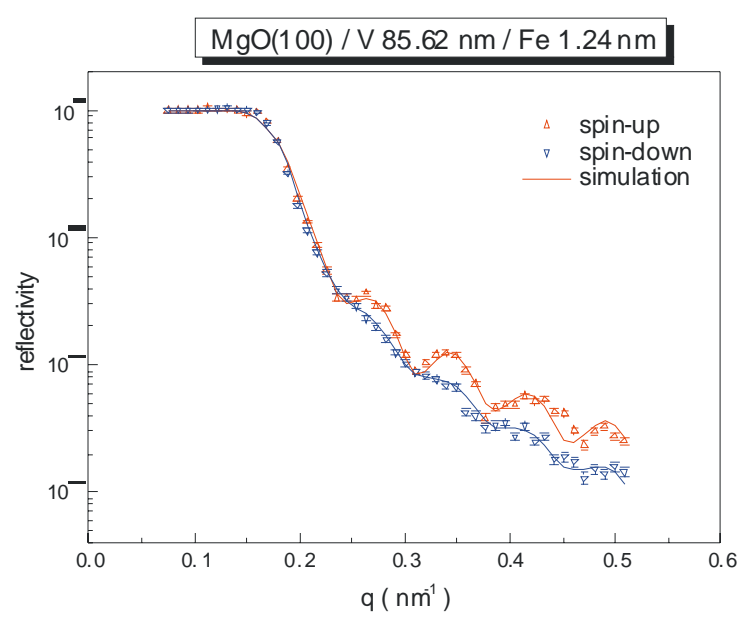

( c )

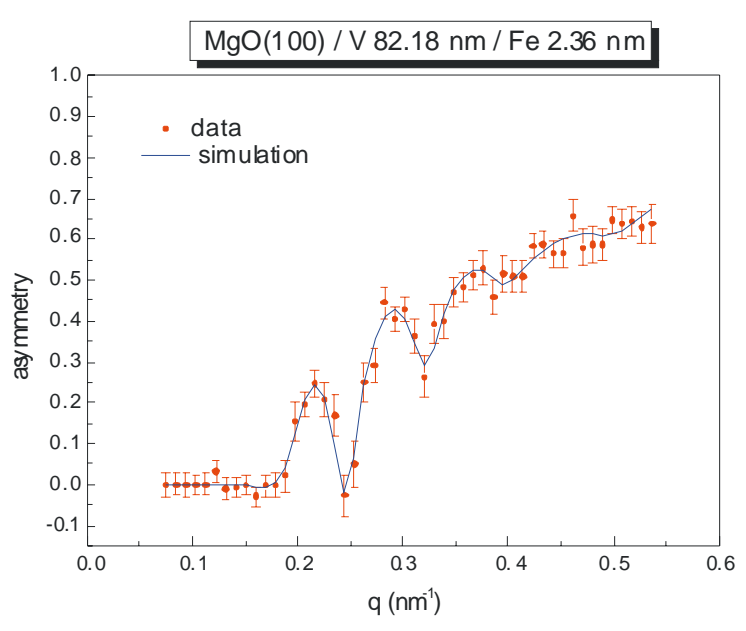

( b )

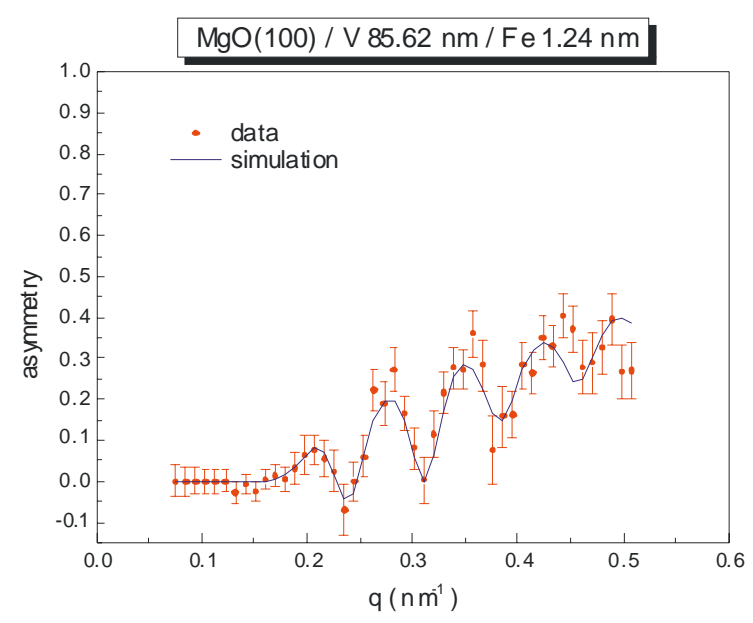

( d )

Figure 5.12 Reflectivity and spin asymmetry curves of the samples with $t_{F e}=$ $2.36 \mathrm{~nm}$ and $t_{\mathrm{Fe}}=1.24 \mathrm{~nm}$ as obtained from in-situ PNR measurements. The reflectivity curves of the spin-up (spin-down) neutrons are given as up-triangles (down-triangles). The spin asymmetry curves are shown as dots. The solid lines are results from simulations. 


\begin{tabular}{|c|c|c|c|c|c|c|}
\hline No. & $\begin{array}{c}\sigma_{\mathrm{MgO}} \\
(\mathbf{n m})\end{array}$ & $\begin{array}{c}\boldsymbol{t}_{\mathrm{V}} \\
(\mathbf{n m})\end{array}$ & $\begin{array}{c}\sigma_{\mathrm{V}} \\
(\mathbf{n m})\end{array}$ & $\begin{array}{c}\boldsymbol{t}_{\mathrm{Fe}} \\
(\mathbf{n m})\end{array}$ & $\begin{array}{c}\sigma_{\mathrm{Fe}} \\
(\mathbf{n m})\end{array}$ & $\begin{array}{c}\mathbf{m}_{\mathrm{Fe}} \\
\left(\mu_{\mathrm{B}} / \mathbf{a t o m}\right)\end{array}$ \\
\hline 1 & 0.2 & 87.1 & 0.2 & 5.36 & 0.2 & 2.11 \\
\hline 2 & 0.2 & 95 & 0.3 & 3.17 & 0.3 & 2.16 \\
\hline 3 & 0.2 & 82.18 & 0.3 & 2.36 & 0.3 & 2.11 \\
\hline 4 & 0.2 & 85.62 & 0.3 & 1.24 & 0.3 & 2.02 \\
\hline
\end{tabular}

Table 5-1 Simulation results obtained from the in-situ PNR measurements. $\sigma_{M g O}, \sigma_{V}$, and $\sigma_{F e}$ are the surface roughnesses of the $\mathrm{MgO}$ substrate, the $V$ and $F e$ layers, respectively. $t_{V}$ and $t_{F e}$ are the thickness of the $V$ and Fe layers. $m_{F e}$ is the magnetic moment of the Fe atoms in the Fe layer.

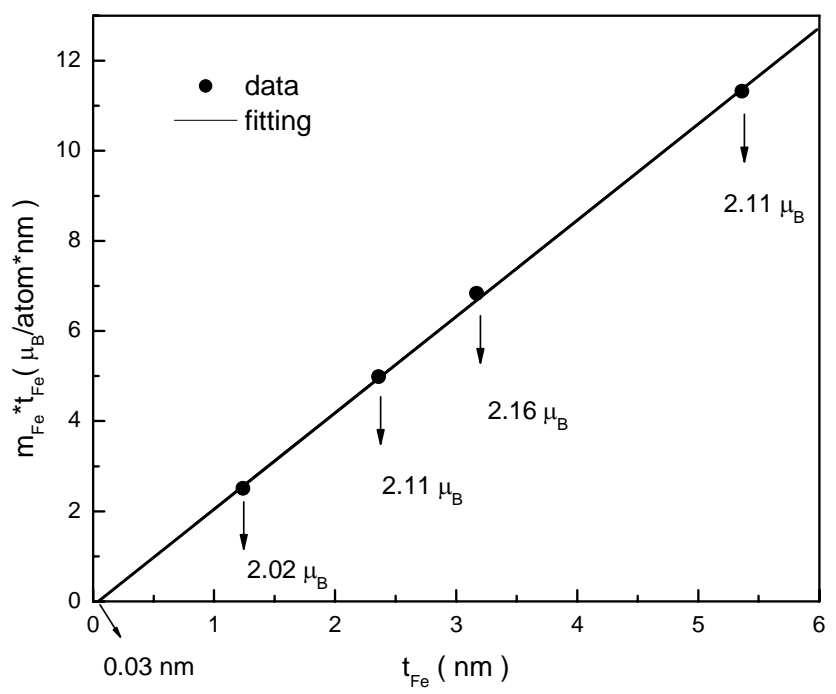

Figure 5.13 The products of the magnetic moment per atom and the Fe film thickness $t_{F e}$ (dots) are plotted versus $t_{F e}$. The solid line is a linear fit to the data points. 


\subsubsection{Discussion}

The linear fit of the product $m_{\mathrm{Fe}}{ }^{*} t_{\mathrm{Fe}}$ as a function of $t_{\mathrm{Fe}}$ shown in Figure 5.13 illustrates again that the magnetic properties of the thin film are composed of a volume and a surface term. It is in agreement with the result obtained from the in-situ MOKE measurements presented before. The magnetic moment of the inner Fe layer of $2.14( \pm 0.04) \mu_{\mathrm{B}} /$ atom was estimated from the slope of the fitted line. This value is the same as that obtained from the exsitu PNR measurements described in Chapter 4. That means, the capping layer does not affect the magnetic properties of the inner part of the Fe layer.

On the other hand, it is obvious that the capping of the Fe film with V influences the magnetization: The experimental results from the in-situ PNR (Figure 5.13) are different from that of the ex-situ PNR (Figure 4.10). They show different intersections at the abscissa in the straight line fit, in spite of the large error bars of the plotted data (the magnetic moment is determined by PNR with an accuracy of $\pm 0.05 \mu_{\mathrm{B}}$; the film thickness accuracy is estimated to be $\pm 0.04 \mathrm{~nm}$ ). Since the fit parameter of the slope in both series (Figure 4.10 and Figure 5.13) is the same and consistent with the expected moment of bulk Fe, the different intersection values clearly indicate an additional effect on the magnetization near the $\mathrm{Fe} / \mathrm{V}$ interface by capping the $\mathrm{Fe}$ film with V, compared to that of the free Fe surface in the in-situ PNR measurements.

The linear fit displayed in Figure 5.13 intersects the abscissa at $0.03( \pm 0.06)$ $\mathrm{nm}$, which corresponds to a reduction of the magnetic moment by $-0.45( \pm 0.9)$ $\mu_{\mathrm{B}}$. It is in agreement with the intersection obtained from the in-situ MOKE measurements (Figure 5.6), which exhibit an intersection of 0.034 $( \pm 0.07) \mathrm{nm}$ $\left(-0.5( \pm 1) \mu_{\mathrm{B}}\right)$. In contrast, the intersection value obtained from the ex-situ PNR measurements is distinctly larger: $0.1( \pm 0.01) \mathrm{nm}\left(-1.5( \pm 0.1) \mu_{\mathrm{B}}\right)$. In order to estimate the difference between the magnetic properties of the free $\mathrm{Fe}$ surface and those at the $\mathrm{Fe} / \mathrm{V}$ interface we combine the results from the fits of both PNR series, keeping in mind the small accuracy of the data on the thin films.

Assuming that the magnetic contribution from each $\mathrm{Fe} / \mathrm{V}$ interface is $\Delta M_{\mathrm{Fe} / V}$ and that from the free Fe surface is $\Delta M_{\text {free }-F e}$, then the following equations can be given:

$$
2 \Delta M_{F e / V}=-1.5( \pm 0.1) \mu_{B} \text { and }
$$




$$
\Delta M_{\mathrm{Fe} / V}+\Delta M_{\text {free_Fe }}=-0.45( \pm 0.9) \mu_{B} .
$$

From these two equations the values of $\Delta M_{F e / V}=-0.75( \pm 0.05) \mu_{B}$ and $\Delta M_{\text {free } F e}=+0.3( \pm 0.9) \mu_{B}$ can be estimated. Here the minus sign denotes the reduction, and the plus sign means enhancement comparing with the magnetic moment of the bulk Fe. We obtain at the $\mathrm{Fe} / \mathrm{V}$ interface a reduction of the magnetic moment by $-0.75( \pm 0.05) \mu_{\mathrm{B}}$, whereas at the free Fe surface the magnetic moment is enhanced by $+0.3( \pm 0.9) \mu_{\mathrm{B}}$.

According to the theoretical calculations [Ohn83, Veg93] an enhanced magnetic moment at the surface was predicted due to reduced coordination number. However, the uncertainty of the experimental PNR result is too large to compare quantitatively the results with theoretical predictions. Yet at least several interesting informations were obtained. The first, from this work it is demonstrated that it is possible to separate the magnetic contributions from the surface and the interface of a thin film by combining the ex-situ PNR with in-situ PNR, but a high intensity neutron source is required to improve the sensitivity; the second, since the two different techniques, both in-situ MOKE and in-situ PNR, show similar results, we can argue that the magnetic enhancement by $+0.3 \mu_{\mathrm{B}}$ at the free Fe surface is more probable.

Similar experiments have been performed by Nawrath et al. [Naw99] on a $\mathrm{Fe} / \mathrm{V}(110)$, a $0.4 \mathrm{~nm}$ intersection was found, corresponding to a reduction of the magnetic moment of $-4.33 \mu_{\mathrm{B}}$ at the $\mathrm{Fe} / \mathrm{V}(110)$ interface. This value is quite different compared with the reduction of the magnetic moment of -0.45 $\mu_{\mathrm{B}}$ obtained from the $\mathrm{Fe} / \mathrm{V}(100)$ in this work. According to theoretical calculations [Izq99] the reduction of the magnetic moment, caused by the induced antiparallel $\mathrm{V}$ magnetic moment, should be larger at the $\mathrm{Fe} / \mathrm{V}(100)$ interface than at the $\mathrm{Fe} / \mathrm{V}(110)$ interface. The reason is that $\mathrm{V}$ has more $\mathrm{Fe}$ nearest neighbors at the (100) interface than at the (110) interface. Thus there is a larger Fe-V hybridization at the (100) interface and therefore the induced magnetization at the $\mathrm{V}(100)$ interface, which is polarized by the adjacent $\mathrm{Fe}$ atoms, is also larger than that at the $\mathrm{V}(110)$ interface. But the results here are reversed. Comparing the sample preparation procedures between these two experiments, a conclusion could be derived that the big difference between these two results might be attributed to the different intermixing at the $\mathrm{Fe} / \mathrm{V}$ interfaces. In the case of the Fe/V(110) sample [Naw99] the V(110) substrate was sputtered and annealed for 120 hours in total in order to clean the crystal. 
This might have increased the roughness of the Vanadium surface. Izquierdo et al. [Izq01] has tried to explain this large reduction of the magnetic moment at the $\mathrm{Fe} / \mathrm{V}(110)$ interface using the model $\mathrm{V}(110) /\left(\mathrm{Fe}_{0.5} \mathrm{~V}_{0.5}\right)_{\mathrm{n}}(\mathrm{n}=1-6)$. However, in the present work 20 -second sputtering was enough to clean the $\mathrm{MgO}(100)$ substrate and the $\mathrm{V}$ buffer layer grown on it was not sputtered at all. So, a larger intermixing of $\mathrm{Fe}$ and $\mathrm{V}$ at this particular $\mathrm{Fe} / \mathrm{V}(110)$ interface [Naw99] is very probable in comparison with our $\mathrm{Fe} / \mathrm{V}(100)$ interfaces. Furthermore, the same kind of Fe/V(110) sample was investigated by Schmitz et al.[Sch04] using X-ray magnetic circular dichroism (XMCD). The induced V magnetic moment, aligned antiparallel to the Fe magnetic moment, is about $0.8 \mu_{\mathrm{B}}$. The Fe magnetic moment is the same as that of the bulk Fe. This result also proves that the large reduction of the magnetic moment of $-4.33 \mu_{\mathrm{B}}$ at $\mathrm{Fe} / \mathrm{V}(110)$ interface as found by Nawrath et al. is only a special case.

\subsection{Conclusion}

The $t_{\mathrm{Fe}} \mathrm{Fe} / \mathrm{V}(100)$ system with different Fe thickness $t_{\mathrm{Fe}}$ was investigated by in-situ MOKE and PNR. These measurements showed that the magnetic properties of the thin films were composed of a volume and a surface term. For the in-situ MOKE measurements the square-shaped hysteresis loops in the region of $0.6 \mathrm{~nm} \leq t_{\mathrm{Fe}} \leq 2 \mathrm{~nm}$ showed that the easy axis was aligned inplane along the [100] direction in this region. When the Fe thickness was smaller than $0.8 \mathrm{~nm}$ the magnetic moment of the Fe films became smaller owing to the reduced Curie temperature of the Fe films. In the in-situ PNR measurements, the magnetic moment of the inner part of the Fe layer was found to be $2.14( \pm 0.04) \mu_{\mathrm{B}} /$ atom, confirming the ex-situ PNR results. The comparison of the results between the in-situ and the ex-situ PNR experiments clearly indicates different intersection values at the abscissa in the linear fits displayed in Figure 4.10 and Figure 5.13. Hence, the capping of the thin Fe film with $\mathrm{V}$ influences the magnetization. Combining the results from both PNR series may help in principle to separate the magnetic contributions from the free $\mathrm{Fe}$ surface and the $\mathrm{Fe} / \mathrm{V}$ interfaces. A rough estimate gives an enhancement of the magnetic moment by $+0.3( \pm 0.9) \mu_{\mathrm{B}} /$ atom at the free $\mathrm{Fe}$ surface.

Comparing the present results with the results obtained by Nawrath et al. [Naw99] and by Schmitz et al. [Sch04] on the Fe/V(110) system it was con- 
cluded that the large reduction of the magnetic moment at the $\mathrm{Fe} / \mathrm{V}(110)$ interface as found by Nawrath et al. is not a generic case. 


\section{Chapter 6 Summary}

The present work emphasized on the PNR method. We further developed it as a powerful tool to study details of the magnetization of an ultrathin film by combining in-situ and ex-situ PNR measurements as a function of the film thickness for the first time. HMI is the only place worldwide where the option of the in-situ PNR is now available. A series of samples of $\mathrm{V} / t_{\mathrm{Fe}} \mathrm{Fe} / \mathrm{V}$ $/ \mathrm{MgO}(100)$ and $t_{\mathrm{Fe}} \mathrm{Fe} / \mathrm{V} / \mathrm{MgO}(100)$ with different Fe thickness $t_{\mathrm{Fe}}$ was prepared and measured using ex-situ PNR, ex-situ XRR, in-situ PNR, and in-situ MOKE. According to the results obtained the product of the magnetic moment per atom and the Fe thickness of ultrathin Fe(100) films was plotted as a function of the Fe thickness. Analyzing these plots the magnetization of the inner part of the Fe layer, the free $\mathrm{Fe}$ surface and the Fe/V interface were derived. In order to perform PNR experiments on uncovered thin films a movable MBE chamber was developed during this work in our group (AG Maletta, HMI). The design of this chamber was based on the experiences gained in earlier in-situ experiments [Naw98a]. This chamber could be moved easily from the lab to the neutron reflectometer.

The critical part of the preparation of the $\mathrm{Fe} / \mathrm{V}(100)$ samples is the preparation of a chemically clean $\mathrm{V}$ substrate layer with a good crystalline quality. It is well known from literature that it is very difficult to clean a Vanadium single crystal [Ada81, Jen82, Naw98]. Therefore, instead of using a V single crystal substrate, a $\mathrm{MgO}(100)$ substrate was selected and a $\mathrm{V}$ buffer layer was prepared on top of it at a rate of $0.01 \mathrm{~nm} / \mathrm{s}$ under a pressure of $2 \times 10^{-10} \mathrm{mbar}$ by thermal evaporation in the MBE chamber from a high purity Vanadium source at room temperature. After the preparation of the $\mathrm{V}$ buffer layer it was annealed at $700 \mathrm{~K}$ for 20 minutes, and afterwards it was covered by an additional ultrathin $3 \mathrm{~nm}$ Vanadium film. This procedure led to a smooth and 
clean $\mathrm{V}(100)$ surface with good crystalline quality as proven by LEED, AES and X-ray rocking scans of the characteristic Bragg peaks, see Figure 3.5, Figure 4.8 and Figure 4.9.

A series of samples of $\mathrm{V} / t_{\mathrm{Fe}} \mathrm{Fe} / \mathrm{V} / \mathrm{MgO}(100)$ with different Fe thickness $t_{\mathrm{Fe}}\left(t_{\mathrm{Fe}}=3.03,2.01,1.53,0.97\right.$ and $\left.0.3 \mathrm{~nm}\right)$ was investigated by ex-situ PNR and XRR. PNR has the advantage to provide magnetic and structural information of the sample, whereas XRR is only sensitive to structural properties. However, compared with neutrons $\mathrm{x}$-rays are more sensitive to surface roughness and capable to distinguish different Vanadium oxides. Thus, with the help of XRR, it was established that $\mathrm{V}_{2} \mathrm{O}_{3}$ was formed only on top of the Vanadium capping layer after the samples were exposed to air. Large roughness values at the surface indicated that the oxide layers were not homogeneous. The oxidation did not penetrate as deep as to reach the $\mathrm{Fe} / \mathrm{V}$ interface which would have influenced the Fe magnetization.

According to the results obtained from ex-situ PNR measurements, the products of the magnetic moment per atom and the Fe thickness of ultrathin $\mathrm{Fe}(100)$ films were plotted as a function of the Fe thickness and shown in Figure 4.10. The linear fit of the data points in the region of $0.97 \leq t_{\mathrm{Fe}} \leq 3.03$ $\mathrm{nm}$ in Figure 4.10 demonstrates that the magnetization is composed of a volume and a surface term. The magnetic moment of the inner part of the $\mathrm{Fe}$ layer is $2.14( \pm 0.02) \mu_{\mathrm{B}} /$ atom as determined from the slope of the fitted line, and the corresponding value at $0 \mathrm{~K}$ was estimated using Bloch law, which was $2.20( \pm 0.02) \mu_{\mathrm{B}}$ /atom agreeing very well with the theoretical result 2.22 $\mu_{\mathrm{B}} /$ atom [Wu99] for bulk Fe. The $0.1( \pm 0.01) \mathrm{nm}$ intersection with the abscissa corresponds to a total reduction of $-1.5( \pm 0.1) \mu_{\mathrm{B}}$. This reduction is composed of the contributions of the two $\mathrm{Fe} / \mathrm{V}$ interfaces and the temperature-dependent size effect. According to the investigation done by Fritzsche et al. [Fri98] on a sample with $0.85 \mathrm{~nm}$ Fe layer on a V(100) substrate, the conclusion can be derived that the size effect in this kind of samples is very small and its influence can be omitted. Thus, in the present work the reduction of $-1.5( \pm 0.1) \mu_{\mathrm{B}}$ mentioned above is mainly due to the contribution of the two Fe/V interfaces. Assuming that both interfaces are equal, then the reduction of the magnetic moment at each Fe/V interface is $-0.75( \pm 0.05) \mu_{\mathrm{B}}$ (about $35 \%$ compared with the Fe bulk value). This value is well in agreement with the theoretical calculations [Ham84, Izq99]. Moreover, a strong deviation from the fit line was found for the data point of the sample with an Fe thick- 
ness of $0.3 \mathrm{~nm}$. This deviation attributed mainly to a reduced Curie temperature. It was supported by following temperature-dependent PNR measurements on this ultrathin film and the results show that the Curie temperature is about $400 \mathrm{~K}$, which is close to room temperature.

For the in-situ MOKE measurements a wedge-shaped sample $t_{\mathrm{Fe}} \mathrm{Fe} / \mathrm{V} /$ $\mathrm{MgO}(100)\left(0.1 \mathrm{~nm} \leq t_{\mathrm{Fe}} \leq 2 \mathrm{~nm}\right)$ was prepared and measured. For Fe thicknesses $t_{\mathrm{Fe}} \geq 0.6 \mathrm{~nm}$ the square-shaped hysteresis loops show that the easy axis is aligned in-plane along the [100] direction. The Kerr intensity was recorded as a function of the Fe layer thickness. It is clearly shown that the Kerr intensities are proportional to the Fe layer thickness in the region of $0.8 \mathrm{~nm} \leq \mathrm{t}_{\mathrm{Fe}} \leq$ $2 \mathrm{~nm}$. The data in this thickness region were fitted by a straight line. Its intersection with the abscissa is $0.034( \pm 0.07) \mathrm{nm}$, corresponding to a total reduction of the magnetic moment of $-0.51( \pm 1) \mu_{\mathrm{B}}$, which is composed of the contributions of the free Fe surface and the Fe/V interface. For Fe thicknesses $t_{\mathrm{Fe}}$ $<0.8 \mathrm{~nm}$ the Kerr intensities deviate from the linear fit line and become smaller. From ex-situ temperature-dependent PNR measurements, this deviation has been proven to be due to the reduced Curie temperature. Furthermore, a plot of the thickness dependence of the coercivity was obtained. It shows a very interesting four-stage behavior when the Fe thickness changes from 0.6 $\mathrm{nm}$ to $2.0 \mathrm{~nm}$.

From the results of the in-situ MOKE measurements it is shown that the magnetization deviates from the linear fit at $300 \mathrm{~K}$ when $t_{\mathrm{Fe}}$ is smaller than $0.8 \mathrm{~nm}$ due to the reduced Curie temperature. So, for the in-situ PNR measurements a series of samples $t_{\mathrm{Fe}} \mathrm{Fe} / \mathrm{V} / \mathrm{MgO}(100)$ with different Fe thickness $t_{\mathrm{Fe}}>0.8 \mathrm{~nm}\left(t_{\mathrm{Fe}}=5.36,3.17,2.36,1.24 \mathrm{~nm}\right)$ was prepared and investigated by in-situ PNR measurements. According to the results obtained from in-situ PNR measurements, the products of the magnetic moment per atom and the thickness of the Fe films were plotted as a function of the Fe thickness and shown in Figure 5.13. The data points in Figure 5.13 were fitted by a straight line. As determined from the slope of the fitted line the magnetic moment of the inner part of the Fe layer is $2.14( \pm 0.04) \mu_{\mathrm{B}} /$ atom, which is the same value as that obtained from the ex-situ PNR measurements. That means the V capping layer does not influence the magnetic properties of the inner Fe layer at all. On the other hand, by comparing the in-situ and ex-situ PNR results (different intersection values at the abscissa) it is obvious that the capping of the thin Fe film with $\mathrm{V}$ influences the magnetization. The deviation from bulk behavior at the free Fe surface may be estimated by the intersection of 
behavior at the free $\mathrm{Fe}$ surface may be estimated by the intersection of $0.03( \pm 0.06) \mathrm{nm}$ with the abscissa in Figure 5.13, which corresponds to a reduction of the magnetic moment by $-0.45( \pm 0.9) \mu_{\mathrm{B}}$ and agrees with the insitu MOKE result. Although the error bar is quite large here comparing with its value, two different techniques (in-situ MOKE and PNR) show similar values. The value of the intersection with the abscissa obtained from the exsitu PNR measurements is $0.1( \pm 0.01) \mathrm{nm}\left(-1.5( \pm 0.1) \mu_{\mathrm{B}}\right)$, thus the magnetization of the free $\mathrm{Fe}$ surface is different from that at the $\mathrm{Fe} / \mathrm{V}$ interface. According to the results obtained from the ex-situ PNR measurements, using the Eq. (5-2), an enhancement of the magnetic moment of $+0.3( \pm 0.9) \mu_{\mathrm{B}}$ at the free Fe surface was estimated. Although here the uncertainty of the experimental result of the magnetic moment at the free Fe surface is too large to compare quantitatively with theoretical calculations, it is demonstrated that one can separate in principle the magnetic contributions from the surface and the interface of thin film by combining the ex-situ PNR with in-situ PNR.

Similar in-situ PNR experiments have been performed by Nawrath et al. [Naw99] on a Fe/ $/(110)$ system and a $0.4 \mathrm{~nm}$ intersection with the abscissa in a plot such as the ones mentioned above was found, corresponding to a reduction of the magnetic moment at the $\mathrm{Fe} / \mathrm{V}(110)$ interface of $-4.33 \mu_{\mathrm{B}}$. This value is quite different compared with the estimated reduction of the magnetic moment of $-0.45 \mu_{\mathrm{B}}$ obtained for the $\mathrm{Fe} / \mathrm{V}(100)$ interface discovered in this work. According to theoretical calculations [Izq99], the reduction of the magnetic moment, caused by an induced antiparallel $\mathrm{V}$ magnetic moment, is expected to be larger at the $\mathrm{Fe} / \mathrm{V}(100)$ interface than at the $\mathrm{Fe} / \mathrm{V}(110)$ interface. The reason is that $\mathrm{V}$ has more Fe nearest neighbors at the (100) interface than at the (110) interface. Thus there is a larger Fe-V hybridization at the (100) interface and therefore the induced magnetization at the $\mathrm{V}(100)$ interface, which is polarized by the adjacent $\mathrm{Fe}$ atoms, is also larger than that at the $\mathrm{V}(110)$ interface. The big difference between the present result and that obtained by Nawrath et al. could be attributed to the different intermixing at the $\mathrm{Fe} / \mathrm{V}$ interfaces. In the case of the $\mathrm{Fe} / \mathrm{V}(110)$ sample prepared by Nawrath et al. a V(110) single crystal substrate was sputtered and annealed for 120 hours in total in order to clean the crystal. This might have increased the large roughness of the Vanadium surface. Izquierdo et al. [Izq01] tried to explain the results obtained by Nawrath et al. using the model $\mathrm{V}(110) /\left(\mathrm{Fe}_{0.5} \mathrm{~V}_{0.5}\right)_{\mathrm{n}}$ $(n=1-6)$ which accounted for intermixing at the interface. However, in the 
present work 20-second sputtering was enough to clean the $\mathrm{MgO}(100)$ substrate and the V buffer layer grown on it was not sputtered at all. So, a larger intermixing of $\mathrm{Fe}$ and $\mathrm{V}$ at this particular $\mathrm{Fe} / \mathrm{V}(110)$ interface [Naw99] is very probable in comparison with our $\mathrm{Fe} / \mathrm{V}(100)$ interfaces. Furthermore, the same kind of $\mathrm{Fe} / \mathrm{V}(110)$ sample was investigated by Schmitz et al.[Sch04] using $\mathrm{x}$-ray magnetic circular dichroism (XMCD). The induced $\mathrm{V}$ magnetic moment, aligned antiparallel to the Fe magnetic moment, was determined to be about $0.8 \mu_{\mathrm{B}}$, and the Fe magnetic moment was the same as that of the bulk Fe. So, this result also proves that the large reduction of the magnetic moment of $-4.33 \mu_{\mathrm{B}}$ at $\mathrm{Fe} / \mathrm{V}(110)$ interface as found by Nawrath et al. is not a generic case.

In short, in the present work an MBE chamber was purposely built in order to perform in-situ PNR experiments. The main emphasis was to show the PNR method as a powerful tool in order to study details of the magnetization of an ultrathin film by combining in-situ and ex-situ PNR measurements for the first time. The aim was to separate and estimate the contributions from the interface, the inner part and the free surface of the film by investigating capped and uncapped films as a function of the film thickness.

A series of samples of $\mathrm{V} / t_{\mathrm{Fe}} \mathrm{nm} \mathrm{Fe} / \mathrm{V} / \mathrm{MgO}(100)$ and $t_{\mathrm{Fe}} \mathrm{nm} \mathrm{Fe} / \mathrm{V}$ $/ \mathrm{MgO}(100)$ as a function of the Fe thickness $t_{\mathrm{Fe}}$ was measured. The experiments clearly indicate that the capping of the thin Fe film with $\mathrm{V}$ changes the total magnetization, whereas it does not affect the magnetic moment of the inner part of the Fe layer (which is that of bulk Fe). Combining both series of PNR experiments demonstrates on the one hand the existence of a reduced magnetic moment at the $\mathrm{Fe} / \mathrm{V}$ interface (estimated to be a reduction of $-0.75( \pm 0.05) \mu_{\mathrm{B}} /$ atom which corresponds to about $35 \%$ of the Fe bulk value). In contrast, an enhanced magnetic moment at the free Fe surface is derived (estimated to be an enhancement of $+0.3( \pm 0.9) \mu_{\mathrm{B}} /$ atom). 


\section{Reference}

[Ada81] D.L. Adams and H.B. Nielsen, Surf. Sci. 107 (1981) 305.

[Alb93] Martin Albrecht, Helmut Fritzsche and Ulrich Gradmann, Surf. Sci. 294 (1993) 1.

[Alv98] P. Alvarado, J. Dorantes-Dávila and G. M. Pastor, Phys. Rev. B 58 (1998) 12216.

[Ara88] J. Araya-Pochet, C. A. Ballentine, and J. L. Erskine, Phys. Rev. B, 38 (1988) 7846.

[Art79] J. R. Arthur, J. Vac. Sci. Technol. 16 (1979) 273.

[Bad86] S. D. Bader, E. R. Moog and P. Grünberg, J. Magn. Magn. Mater. 53 (1986) L295.

[Bad94] S. D. Bader and J. L. Erskine, Magneto-Optical Effects in Ultrathin Magnetic Structures, in ULTRATHIN MAGNETIC STRUCTURES II, J. A. C. Bland and B. Heinrich (Eds.), Springer-Verlag Berlin (1994).

[Bau58] E. Bauer, Z. Kristallogr., 110 (1958) 37.

[Bes75] K. Besocke and H. Wagner, Surf. Sci. 52 (1975) 653.

[Bie97] J. Biener, R. J. Madix, M. Baeumer, experimental progress reports 7 (1997) 363.

[Bla94] J. A. C. Bland, Polarized Neutron Reflection, in ULTRATHIN MAGNETIC STRUCTURES I, J. A. C. Bland and B. Heinrich (Eds.), Springer-Verlag Berlin (1994).

[Bra] C. Braun, The used software Parratt32 was developed by C. Braun for BENSC, HMI.

[Car85] P.F. Carcia, A.D. Meinhaldt, A. Suna, Appl. Phys. Lett. 47 (1985) 178.

[Cho79] A. Y. Cho, J. Vac. Sci. Technol. 16 (1979) 275.

[Chr95] M. J. Christensen, R. Feidenhans'I and M. Nielsen, Vacuum 46 (1995) 1113. 
[Cla85] L. J. Clarke, Surface Crystallography, John Wiley \& Sons Ltd. (1985).

[Coe95] R. Coehoorn, J. Magn. Magn. Mater. 151 (1995) 341.

[Dob94] Ludwik Dobrzynski and Konrad Blinowski, NEUTRONS AND SOLID STATE PHYSICS, Malcolm Cooper (Ed.), Ellis Horwood Limited (1994).

[Dud96] L.-C. Duda, P. Isberg, S. Mirbt, J.-H. Guo, b. Hjörvarsson, J. Nordgren and P. Granberg, Phys. Rev. B 54 (1996) 10393.

[Due89] W. Duerr, M. Taborelli, O. Paul, R. Germar, W. Gudat, D. Pescia and M. Landolt, Phys. Rev. Lett. 62 (1989) 206.

[Du101] F. Dulot, P. Turban, B. Kierren, J. Eugène, M. Alnot, S. Andrieu, Surf. Sci. 473 (2001) 172.

[Elm89] H. J. Elmers, G. Liu, and U. Gradmann, Phys. Rev. Lett. 63 (1989) 566.

[Eng91] B.N. Engel, C.D. England, R.A. Van Leeuwen, M.H. Wiedmann, C.M. Falco, Phys. Rev. Lett. 67 (1991) 1910.

[Ert85] G. Ertl, J. Kueppers, Low Energy Electrons and Surface Chemistry, VCH Verlagsgesellschaft $\mathrm{mbH}$ Weinheim (1985).

[Fer99] Claude Fermon, Frédéric Ott and Alain Menelle, Neutron Reflectometry in X-Ray and Neutron Reflectivity: Principles and Applications, Springer-Verlag Berlin (1999).

[Fle95] Ron Fleming, Auger Electron Spectrometry Theory Tutorial, Http://www.cea.com/cai/augtheo/caiatheo.htm.

[Foo83] J.S. Foord, Surf. Sci. 129 (1983) 79.

[Fra49] F.C. Frank and J.H. van der Merwe, Proc. R. Soc. A 198 (1949) 205.

[Fre85] A. J. Freeman, C. L. Fu, S. Ohnishi, and M. Weinert, Electronic and magnetic structure of solid surfaces, in polarized electrons in surface physics, R. Feder, (Eds.), World Scientific, Singapore, 1985, 3. 
[Fre87] A. J. Freeman, C. L. Fu, J. I. Lee, T. Oguchi, predictions of magnetism at interfaces and superlattices, in Physics of Magnetic Materials, M. Takahashi, et al, (Eds.) Worlk Scientific, Singapore, 1987, 221.

[Fri95] H. Fritzsche, Struktur und Magnetismus dünner Eisenund Kobalt-Epitaxieschichten, Dissertation, TU Clauthsthal (1995).

[Fri98] H. Fritzsche, T. Nawrath, H. Maletta, H. Lauter, Physica B 241-243 (1998) 707.

[Fu85] C. L. Fu, A. J. Freeman, T. Oguchi, Phys. Rev. Lett. 54 (1985) 2700.

[Fuc96] P. Fuchs, K. Totland and M. Landolt, Phys. Rev. B 53 (1996) 9123.

[Gie02] M. Gierlings, Magnetic properties of exchange-coupled antiferromagnetic/ferromagnetic multilayers studied by Polarized Neutron Reflectometry and Low Temperature Nuclear Orientation, Dissertation, FU Berlin (2002).

[Gra68] U. Gradmann, J. Müller, Phys. Status Solidi 27 (1968) 313.

[Gra93] U. Gradmann, Magnetism in ultrathin transition metal films in Handbook of magnetic materials, Vol. 7 K. H. J. Buschow, (Eds.) Elsevier Science Publishers, Amsterdam, 1993.

[Gra98] P. Granberg, P. Isberg, E.B. Svedberg, B. Hjörvarsson, P. Nordblad, R. Wäppling, J. Magn. Magn. Mater 186 (1998) 154.

[Ham84] N Hamada, K. Terakura and A. Yanase, J. Phys. F: Met. Phys. 14 (1984) 2371.

[Har95] G. R. Harp and S. S. Parkin, Phys. Rev. B 51 (1995) 3293.

[Hen70] M. Henzler, Surf. Sci. 19 (1970) 159.

[Hos84] Nobuyoshi Hosoito, Henji Kawasguchi, Teruya Shinjo, Toshio Takada and Yasuo Endoh, J. Phys. Soc. Jpn. 53 (1984) 2659.

[Hov86] M. A. Van Hove, W. H. Weinber and C. -M. Chan, LowEnergy Electron Diffraction, Springer-Verlag Berlin 
(1986).

[How01] Jonathan R. Howse, Roland Steitz, Michael Pannek, Paul Simon, Dirk W. Schubert and Gerhard H. Findenegg, Phys. Chem. Chem. Phys., 3 (2001) 4044.

[Ige00] T. Igel, R. Pfandzelter, H. Winter, Solid State Communications 116 (2000) 477.

[Izq01] J. Izquierdo, R. Robles, A. Vega, M. Talanana, C. Demangeat, Phys. Rev. B 64 (2001) 060404-1.

[Izq99] J. Izquierdo, A. Vega, O. Elmouhssine, H. Dreyssé and C. Demangeat, Phys. Rev. B 59 (1999) 14510.

[Jen82] V. Jensen, J.N. Andersen, H.B. Nielsen and D.L. Adams, Surf. Sci. 116 (1982) 66.

[Koh92] J. Kohlhepp, H. J. Elmers, S Cordes, and U. Gradmann, Phys. Rev. B, 45 (1992) 12287.

[Kre00] G. Krenn, C. Eibl, W. Mauritsch, E.L.D. Hebenstreit, P. Varga, A. Winkler, Surf. Sci. 445 (2000) 343.

[Kri95] Th. Krist, C. Pappas, Th. Keller, F. Mezei, Physica B 213\&214 (1995) 939.

[Lie69] L. N. Liebermann, D. R. Fredkin, and H. B. Shore, Phys. Rev. Lett. 22 (1969) 539.

[Lie70] L. N. Liebermann, J. Clinton, P. M. Edwards, and J. Mathon, Phys. Rev. Lett. 25 (1970) 232.

[Lin94] Ch. Linsmeier, Auger electron spectroscopy, in Surface Science Techniques, J. M. Walls and R. Smith (Eds), Elsevier Science Ltd (1994).

[Liu91] C. Liu and S. D. Bader, J. Magn. Magn. Mater. 93 (1991) 307.

[Maj99] C.F. Majkrzak, J. Neutron Research 7 (1999) 159.

[Mar95] P. Martin, A. Vega, C. Demangeat, H. Dreyssé, J. Magn. Magn. Mater. 148 (1995) 177.

[Mez95] F. Mezei, R. Golub, F. Klose and H. Toews, Physica B 213\&214 (1995) 898.

[Moo85] E. R. Moog and S. D. Bader, Superlattices and Microstructures, 1 (1985) 543. 
[Mos98] A. Moser, U. Krey, A. Paintner, B. Zellermann, J. Magn. Magn. Mater. 183 (1998) 272.

[Naw00] T. Nawrath, H. Fritzsche, H. Maletta, J. Magn. Magn. Mater. 212 (2000) 337.

[Naw98] T. Nawrath, H. Fritzsche, H. Maletta, Surf. Sci. 414 (1998) 209.

[Naw98a] T. Nawrath, In-situ-Neutronenreflektometrie und KerrMagnetometrie an ultradünnen Eisenschichten auf Vanadium(110), Dissertation, TU Berlin (1998).

[Naw99] T. Nawrath, H. Fritzsche, F. Klose, J. Nowikow and H. Maletta, Phys. Rev. B 60 (1999) 9525.

[Ned85] P. O. J. Nederpel and J. W. D. Martens, Rev. Sci. Instrum. 56(5) (1985) 687.

[Née54] L. Néel, J. Phys. Radium 15, (1954) 225.

[Nix] Roger Nix, An Introduction to Surface Chemistry, Http://www.chem.qmw.ac.uk/surfaces/scc.

[Ohn83] S. Ohnishi, A. J. Freeman and M. Weinert, Phys. Rev. B 28 (1983) 6741.

[Pan80] M. B. Panish, Science 208 (1980) 916.

[Par54] L. G. Parratt, Phys. Rev. 95 (1954) 359.

[Pou97] P. Poulopulos, P. Isberg, W. Platow, W. Wisny, M. Farle, B. Hjörvarsson, K. Baberschke, J. Magn. Magn. Mater. 170 (1997) 57.

[Rau89] C. Rau, Appl. Phys. A 49 (1989) 579.

[Rhe77] G.E. Rhead, Surf. Sci. 68 (1977) 20.

[Sch01] A. Scherz, H. Wende, P. Poulopoulos, J. Lindner, K. Baberschke, P. Blomquist, R. Wäppling, F. Wilhelm, N. B. Brookes, Phys. Rev. B 64 (2001) 180407(R).

[Sch04] D. Schmitz, J. Hauschild, P. Imperia, Y. T. Liu, H. Maletta, J. Magn. Magn. Mater. 269 (2003) 89.

[Sch93] F. O. Schumann and J. A. C. Bland, J. Appl. Phys. 73 
(1993) 5945.

[Sch97] Detlef Schhmitz, Magnetic properties of ultrathin films studied by circular dichroism in x-ray spectroscopies, Berichte des Forschungszentrums Juelich, ISSN 09442952.

[Se101] R. Sellmann, Spin-Reorientierungsübergang ultradünner epitaktischer Kobalt-Schichten, Dissertation, TU-Berlin (2001).

[She02] J. Shen, J. Kirschner, Surface Science 500 (2002) 300.

[Sie92] H. C. Siegman, J. Phys.: Condens. Matter 4 (1992) 8395.

[Spi00] D. Spišák and J. Hafner, Phys. Rev. B 61 (2000) 4160.

[Sto95] D. Stoeffler, F. Gautier, J. Magn. Magn. Mater. 147 (1995) 260.

[Tam90] E. Tamura, R. Feder, G. Waller and U. Gradmann, Phys. Status Solidi B 157 (1990) 627.

[Tom97] M. A. Tomaz, W. J. Antel Jr, W. L. O'Brien and G. R. Harp, J. Phys.: Condens. Matter 9 (1997) L179.

[Tur01] P. Turban, F. Dulot, B. Kierren, S. Andrieu, Appl. Surf. Sci. 177 (2001) 282.

[Veg91] A. Vega, A. Rubio, L. C. Balbas, J. Dorantes-Davila, S. Bouarab, C. Demangeat, A. Mokrani and H. Dreyssé, J. Appl. Phys. 69 (1991) 4544.

[Veg93] A. Vega, L. C. Balbás, H. Nait-Laziz, C. Demangeat and H. Dreyssé, Phys. Rev. B 48 (1993) 985.

[Veg95] A. Vega, C. Demangeat, H. Dreyssé, A. Chouairi, Phys. Rev. B 51 (1995) 11546.

[Wa194] T. G. Walker and H. Hopster, Phys. Rev. B 49 (1994) 7687.

[We199] A. A. van Well, Neutron Reflectometry, in NEUTRON SCATTERING INTRODUCTORY COURSE TO ECNS'99, G. Kádár and L. Rosta (Eds.).

[Wu99] R. Wu and A.J. Freeman, J. Magn. Magn. Matter. 200 (1999) 498. 
[Xu98] Y. B. Xu, E. T. M. Kernohan, D. J. Freeland, A. Ercole, M. Tselepi, and J. A. C. Bland, Phys. Rev. B, 58 (1998) 890.

[Zab94] H. Zabel, Appl. Phys. A 58 (1994) 159. 


\section{Acknowledgements}

First I would like to thank Prof. Dr. H. Maletta for giving me the opportunity to do my doctoral work at the Hahn-Meitner-Institut Berlin. I am very grateful for his continuous support in these three years.

I am very appreciative of Prof. Dr. C. Thomsen for all his help and support in the Technischen Universität Berlin and spending his time to assess this thesis.

I am very grateful to Dr. J. Hauschild for his supports in this work. He spent much time on building the MBE preparation chamber in order to do the in-situ PNR measurements. I will never forget the pleasure time we spend together.

I am greatly indebted to Dr. H. Fritzsche for his meticulously support since I came here. With his help I could find many pleasure in the science work. And I will also never forget the first German word "Mahlzeit" that I learned from him.

I also want to make an acknowledgement for Dr. D. Schmitz. He always gives his hands when I need help. From him I learned how to do research work with MCXD during the two months beam time in BESSY II.

Many thanks to Dr. M. Gierlings for spending much time to help me correct the grammar mistakes made in my thesis. Whenever I need help she is always there. I will never forget our fruitful discussion on physics and also her "wake up tee".

I would like to thank Mr. S. Bonn for his strictly correction for my spoken English and useful discussions on physics.

I want to thank Dr. R. Steitz and Dr. H. Bleif for the useful discussion about the $\mathrm{X}$-ray measurements in this work.

I want to say thanks to Dr. H.A. Graf for his help and care in HMI.

I am indebted to Prof. Dr. M. Steiner and Prof. Dr. F. Mezei for offering me a chance to come to Germany.

Here I would like to say thanks to all of colleagues in HMI. Thanks them for nice and inspiring working atmosphere.

Last but not least I thank my family for their selflessness support and care! 


\section{Publications:}

J. Hauschild, H. Fritzsche, S. Bonn, Y. Liu,

Determination of the temperature dependence of the coercivity in $\mathrm{Fe} / \mathrm{Cr}$ (110) multilayers

Appl. Phys. A 74 [Suppl.] (2002) S1541.

D. Schmitz, J. Hauschild, P. Imperia, Y. T. Liu, H. Maletta,

Element-specific investigation of the magnetization of ultrathin Fe on a

$\mathrm{V}(110)$ single crystal

J. Magn. Magn. Mater., 269 (2004) 89.

Y. T. Liu, H. Fritzsche, J. Hauschild, H. Maletta,

Polarized neutron reflectometry study of thin Fe films prepared on $\mathrm{V}(100)$

Physica B, (accepted). 


\section{Curriculum vitae}

Name

Sex

Date of birth

Place of birth

Nationality

Marital status

Eduaction

Jul. 1991 - Jul. 1995

Jul. 1994 - Jul. 1995

Jul. 1997 - Dec. 1999

Since Jan. 2001

Professional Experience

Jul. 1995 - May 2000

Jun. 2000 - Dec. 2000
Yuntao Liu

Male

Feb. 4th, 1972

Liaoning province, China

The People's Republic of China

married

the Physics Department of Jilin University

the speciality of solid-state physics in the Physics Department of Jilin University Degree: Bachelor of Science the speciality of condensed-state physics in China Institute of Atomic Energy Degree: Master of Science PhD. Student at the Hahn-Meitner-Institut Berlin, AG Prof. Dr. H. Maletta

research assistant in China Institute of Atomic Energy guest scientist in Hahn-Meitner-Institut 


\section{Selbständigkeitserklärung}

Hiermit erkläre ich, die vorliegende Arbeit selbständig verfaßt zu haben und nur die angegebene Literatur und Hilfsmittel benutzt zu haben.

Yuntao Liu

08. Dez. 2003 